\title{
Deuterium fractionation and the degree of ionisation in massive clumps within infrared dark clouds $\mathbf{s}^{\star \star \star}$
}

\author{
O. Miettinen ${ }^{1}$, M. Hennemann ${ }^{2}$, and H. Linz ${ }^{3}$ \\ 1 Department of Physics, PO Box 64, 00014 University of Helsinki, Finland \\ e-mail: oskari.miettinen@helsinki.fi \\ 2 Service d'Astrophysique, Laboratoire AIM, CEA - CNRS - Université Paris Diderot, CEA-Saclay, 91191 Gif-sur-Yvette Cedex, \\ France \\ 3 Max-Planck-Institut für Astronomie, Königstuhl 17, 69117 Heidelberg, Germany
}

Received 4 May 2011 / Accepted 29 August 2011

\begin{abstract}
Context. Massive clumps associated with infrared dark clouds (IRDCs) are promising targets for studying the earliest stages of highmass star and cluster formation.

Aims. We aim to determine the degrees of CO depletion, deuterium fractionation, and ionisation in a sample of seven massive clumps associated with IRDCs.

Methods. The APEX telescope was used to observe the $\mathrm{C}^{17} \mathrm{O}(2-1), \mathrm{H}^{13} \mathrm{CO}^{+}(3-2)$, $\mathrm{DCO}^{+}(3-2), \mathrm{N}_{2} \mathrm{H}^{+}(3-2)$, and $\mathrm{N}_{2} \mathrm{D}^{+}(3-2)$ transitions towards the clumps. The spectral line data were used in conjunction with the previously published and/or archival (sub)millimetre dust continuum observations of the sources. The data were used to derive the molecular column densities and fractional abundances for the analysis of deuterium fractionation and ionisation.

Results. The CO molecules do not appear to be significantly depleted in the observed clumps. The $\mathrm{DCO}^{+} / \mathrm{HCO}^{+}$and $\mathrm{N}_{2} \mathrm{D}^{+} / \mathrm{N}_{2} \mathrm{H}^{+}$ column density ratios are about $0.0002-0.014$ and $0.002-0.028$, respectively. The former ratio is found to decrease as a function of gas kinetic temperature. A simple chemical analysis suggests that the lower limit to the ionisation degree is in the range $x(\mathrm{e}) \sim 10^{-8}-10^{-7}$, whereas the estimated upper limits range from a few $10^{-6}$ up to $\sim 10^{-4}$. Lower limits to $x(\mathrm{e})$ imply that the cosmic-ray ionisation rate of $\mathrm{H}_{2}$ lies between $\zeta_{\mathrm{H}_{2}} \sim 10^{-17}-10^{-15} \mathrm{~s}^{-1}$. These are the first estimates of $x(\mathrm{e})$ and $\zeta_{\mathrm{H}_{2}}$ towards massive IRDCs reported so far. Some additional molecular transitions, mostly around 216 and $231 \mathrm{GHz}$, were detected towards all sources. In particular, IRDC 18102-1800 MM1 and IRDC 18151-1208 MM2 show relatively line-rich spectra. Some of these transitions might be assigned to complex organic molecules, although the line blending hampers the identification. The $\mathrm{C}^{18} \mathrm{O}(2-1)$ transition is frequently seen in the image band.

Conclusions. The finding that $\mathrm{CO}$ is not depleted in the observed sources conforms to the fact that they show evidence of star formation activity, which is believed to release CO from the icy grain mantles back into the gas phase. The observed degree of deuteration is lower than in low-mass starless cores and protostellar envelopes. Decreasing deuteration with increasing temperature is likely to reflect the clump evolution. On the other hand, the association with young high-mass stars could enhance $\zeta_{\mathrm{H}_{2}}$ and $x(\mathrm{e})$ above the levels usually found in low-mass star-forming regions. On the scale probed by our observations, ambipolar diffusion cannot be a main driver of clump evolution unless it occurs on timescales $\gg 10^{6} \mathrm{yr}$.
\end{abstract}

Key words. astrochemistry - stars: formation - ISM: abundances - ISM: clouds - ISM: molecules - radio lines: ISM

\section{Introduction}

Most, if not all, infrared dark clouds (IRDCs; Pérault et al. 1996; Egan et al. 1998) studied so far are fragmented into clumpy structures. Some of the clumps within IRDCs that have been studied with high-resolution interferometric observations are found to contain still denser cores, indicating that the clumps have further fragmented into smaller pieces (Rathborne et al. 2007, 2008; Zhang et al. 2009; Beuther \& Henning 2009; Wang et al. 2011) ${ }^{1}$.

\footnotetext{
* This publication is based on data acquired with the Atacama Pathfinder Experiment (APEX) under programmes 081.F-9823A, 082.F-9320A, 083.C-0230A, and 085.F-9321A. APEX is a collaboration between the MPIfR, the European Southern Observatory, and the Onsala Space Observatory.

$\star \star$ Appendices A and B are available in electronic form at http://www . aanda.org

1 We use the term "clump" to describe a density enhancement within an IRDC with a size of about $\sim 0.5-1 \mathrm{pc}$ and a mass of the order of $10^{2}-10^{3} M_{\odot}$. The term "core" is also often used for these objects.
}

Massive clumps in IRDCs are promising targets for studying the earliest stages of high-mass star- and star-cluster formation (e.g., Kauffmann \& Pillai 2010, and references therein). (Sub)millimetre dust continuum maps of IRDCs have shown that the clumps within them have typical radii, masses, beam-averaged $\mathrm{H}_{2}$ column densities, and volume-averaged $\mathrm{H}_{2}$ number densities of $\sim 0.1-0.5 \mathrm{pc}, \sim 10^{2}-10^{3} M_{\odot}, \gtrsim 10^{22} \mathrm{~cm}^{-2}$, and $\sim 10^{4}-10^{5} \mathrm{~cm}^{-3}$, respectively (e.g., Rathborne et al. 2006; Parsons et al. 2009; Vasyunina et al. 2009; Rygl et al. 2010; Miettinen \& Harju 2010). Molecular spectral-line observations towards these clumps have shown the typical gas kinetic temperature to lie in the range $T_{\mathrm{kin}} \approx 10-20 \mathrm{~K}$ (Carey et al. 1998; Teyssier et al. 2002; Sridharan et al. 2005; Pillai et al. 2006b; Sakai et al. 2008, hereafter SSK08; Zhang et al. 2011; Devine et al. 2011; Ragan et al. 2011). Spectral energy distributions of clumps within IRDCs yield dust temperature values of $T_{\text {dust }} \approx 10-50 \mathrm{~K}$ (e.g., Rathborne et al. 2010; Henning et al. 2010). The star-formation process within some of these sources manifests itself through embedded infrared 
emission (e.g., Chambers et al. 2009; Ragan et al. 2009; Henning et al. 2010), outflows (Beuther \& Sridharan 2007; Fallscheer et al. 2009; Sanhueza et al. 2010), and $\mathrm{H}_{2} \mathrm{O}$ and $\mathrm{CH}_{3} \mathrm{OH}$ maser emission (Pillai et al. 2006a; Ellingsen 2006; Wang et al. 2006). Hot cores (Beuther et al. 2005a, 2006; Rathborne et al. 2007, 2008) and UC HII regions (Battersby et al. 2010) have also been discovered within IRDCs. These are clear signs of high-mass star-formation activity. On the other hand, some of the massive clumps are dark at mid-infared (MIR) and do not show any other evidence of ongoing star formation (e.g., G024.61-00.33 MM2, Rygl et al. 2010; and G028.23-00.19 GLM1, Battersby et al. 2010). These sources are candidates for being the high-mass prestellar or precluster objects and are promising targets for the studies of the initial conditions of massive star-formation.

In this paper we aim to further constrain the physical and chemical characteristics of massive clumps associated with IRDCs. We study the degrees of $\mathrm{CO}$ depletion and deuterium fractionation, and the fractional ionisation in a sample of seven clumps. So far, only a few studies have been carried out to investigate molecular deuteration in IRDCs (Pillai et al. 2007; 2010; Fontani et al. 2011; Pillai et al. 2011). The level of deuteration has the potential to be used as an evolutionary indicator (e.g., Crapsi et al. 2005; Emprechtinger et al. 2009; Fontani et al. 2011) and as such it is useful to study it in IRDCs. The ionisation degree, $x(\mathrm{e})$, and the cosmic-ray ionisation rate of $\mathrm{H}_{2}, \zeta_{\mathrm{H}_{2}}$, are also essential knowledge towards an understanding of the initial conditions of star formation (e.g., Caselli et al. 1998). Fractional ionisation determines the coupling between the gas and magnetic field, and can thus play a role in the dynamical evolution of a star-forming object. In addition, the ionisation fraction has an influence on the gas-phase chemical reactions, and thus assessing the ionisation structure of a source helps to better understand its overall chemistry. Because estimates of $x(\mathrm{e})$ and $\zeta_{\mathrm{H}_{2}}$ in massive clumps within IRDCs have not been reported so far, constraining these parameters through observations seems particularly worthwhile.

This paper is organised as follows. The observations, datareduction procedures, and the source sample are described in Sect. 2. The direct observational results are presented in Sect. 3. In Sect. 4 we describe the analysis and present the results of the physical and chemical properties of the clumps. In Sect. 5 we discuss our results, and in Sect. 6 we summarise the main conclusions of this study.

\section{Source selection, observations, and data reduction}

\subsection{Source selection}

For this study we selected six clumps from the previous studies by Beuther et al. (2002a), Sridharan et al. (2005), and Rathborne et al. (2006), and one clump, ISOSS J18364-0221 SMM12, previously studied by Birkmann et al. (2006) and Hennemann et al. (2009). We selected sources with a high mass inside a typical clump radius of $\lesssim 0.5 \mathrm{pc}$ (revised masses from the above reference studies are $\sim 60-360 M_{\odot}$; see Sect. 4.1.3), and which are likely to represent different evolutionary stages of (high-mass) star formation: two of the clumps are clearly associated with Spitzer 8- $\mu \mathrm{m}$ point-sources and five appear dark in the Spitzer $8-\mu \mathrm{m}$ images. Moreover, three of the clumps are associated with

\footnotetext{
${ }^{2}$ Hereafter, we use the abbreviated source names, such as J18364 SMM1 etc.
}

$\mathrm{H}_{2} \mathrm{O}$ and/or $\mathrm{CH}_{3} \mathrm{OH}$ masers. As will be discussed later, the youngest sources in our sample are likely to be G015.05 MM1 and G015.31 MM3, whereas I18102 MM1 appears to be in the most advanced stage of evolution. In this way, we were aiming to investigate if there are any variations or evolutionary trends in the clump physical and chemical properties among the sample.

To gather the source sample, one criterion was that the source distance is not very long (revised values are $\sim 2.5-3.5 \mathrm{kpc}$; see Sect. 4.1.1 and references therein) to achieve a reasonable spatial resolution with the single-dish telescope. For example, 20" $-30^{\prime \prime}$ corresponds to $\sim 0.24-0.51 \mathrm{pc}$ at the cloud distances, i.e., the spatial resolution is comparable to the clump sizes. We selected sources for which dust continuum data at (sub)mm wavelengths are available to derive the $\mathrm{H}_{2}$ column density needed in the derivation of molecular fractional abundances. Also, to derive reliable physical parameters of the sources, they were supposed to have known gas kinetic temperature or dust temperature. The source list is given in Table 1 and the sources are discussed in more detail in Appendix B.

\subsection{APEX molecular-line observations}

In this subsection we describe the molecular-line observations carried out with the Atacama Pathfinder Experiment (APEX) 12-m telescope at Llano de Chajnantor (Chile). The observed lines, their spectroscopic properties, and observational parameters are listed in Table 2.

\subsection{1. $\mathrm{C}^{17} \mathrm{O}(2-1)$}

The $\mathrm{C}^{17} \mathrm{O}(2-1)$ observations towards all the sources except J18364 SMM1 were carried out on 25 March 2008 with APEX during the observational campaign for the Science Verification (SV) of the Swedish Heterodyne Facility Instrument (SHFI or SHeFI; Belitsky et al. 2007; Vassilev et al. 2008a). The source J18364 SMM1 was observed on 26 April 2009. As frontend we used APEX-1 of the SHFI (Vassilev et al. 2008b). APEX-1 operates in single-sideband (SSB) mode using sideband separation mixers, and it has a sideband rejection ratio $>10 \mathrm{~dB}$. The centre frequency for the image band is given by $v_{\text {image }}=v_{\text {cen }} \pm 12 \mathrm{GHz}$, where $v_{\text {cen }}$ is the passband centre frequency, and the \pm refers to the image band corresponding to the upper or lower sideband (USB or LSB), respectively. The backend was the Fast Fourier Transfrom Spectrometer (FFTS; Klein et al. 2006) with a $1 \mathrm{GHz}$ bandwidth divided into 16384 spectral channels during SV and 8192 channels during observations towards J18364 SMM1.

The observations were performed in the wobbler-switching (WS) mode with a $150^{\prime \prime}$ azimuthal throw (symmetric offsets) and a chopping rate of $0.5 \mathrm{~Hz}$. The telescope pointing and focusing were checked by continuum scans on the planet Jupiter and the pointing was found to be accurate to $\sim 3^{\prime \prime}$. Calibration was made by the chopper-wheel technique, and the intensity scale given by the system is $T_{\mathrm{A}}^{*}$, the antenna temperature corrected for atmospheric attenuation.

The spectra were reduced using the CLASS90 programme of the IRAM's GILDAS software package ${ }^{3}$. The individual spectra were averaged and the resulting spectra were Hanning-smoothed to increase the signal-to-noise $(\mathrm{S} / \mathrm{N})$ ratio of the data. A firstor third-order polynomial was applied to correct the baseline in the resulting spectra. In one case (G015.05 MM1) a polynomial baseline of order six had to be applied because there appeared to be some emission in the wobbler off-beams, which resulted in

\footnotetext{
${ }^{3}$ http://www.iram.fr/IRAMFR/GILDAS
} 
Table 1. Source list.

\begin{tabular}{|c|c|c|c|c|c|c|c|}
\hline Source & $\begin{array}{c}\alpha_{2000.0} \\
{[\mathrm{~h}: \mathrm{m}: \mathrm{s}]}\end{array}$ & $\begin{array}{l}\delta_{2000.0} \\
{\left[:^{\circ}::^{\prime \prime}\right]}\end{array}$ & $\begin{array}{c}l \\
{\left[{ }^{\circ}\right]}\end{array}$ & $\begin{array}{c}b \\
{\left[{ }^{\circ}\right]}\end{array}$ & $\begin{array}{c}R_{\mathrm{GC}} \\
{[\mathrm{kpc}]}\end{array}$ & $\begin{array}{c}d \\
{[\mathrm{kpc}]}\end{array}$ & Spitzer $8 \mu \mathrm{m} / 24 \mu \mathrm{m}$ \\
\hline IRDC 18102-1800 MM1 & 181311.0 & -175959 & 12.624 & -0.016 & 5.8 & $2.7_{-0.6}^{+0.5}$ & point/point \\
\hline G015.05+00.07 MM1 & 181750.4 & -155338 & 15.006 & 0.009 & 5.9 & $2.6_{-0.6}^{+0.5}$ & dark/dark \\
\hline IRDC 18151-1208 MM2 & 181750.4 & -120755 & 18.319 & 1.792 & 5.9 & $2.7_{-0.5}^{+0.4}$ & $\operatorname{dark} /-^{a}$ \\
\hline G015.31-00.16 MM3 & 181845.3 & -154158 & 15.281 & -0.093 & 5.6 & $3.0_{-0.5}^{+0.4}$ & dark/dark \\
\hline IRDC 18182-1433 MM2 & 182114.9 & -143306 & 16.577 & -0.081 & 5.1 & $3.5_{-0.4}^{+0.4}$ & point/point \\
\hline IRDC 18223-1243 MM3 & 182508.3 & -124527 & 18.605 & -0.075 & 5.2 & $3.5_{-0.4}^{+0.3}$ & dark/point \\
\hline ISOSS J18364-0221 SMM1 & 183636.0 & -022145 & 29.135 & 2.220 & 6.3 & $2.5_{-0.4}^{+0.4}$ & dark/point \\
\hline
\end{tabular}

Notes. Columns (2)-(7) give the equatorial and galactic coordinates $\left[(\alpha, \delta)_{2000.0}\right.$ and $(l, b)$, respectively], the source galactocentric distance $\left(R_{\mathrm{GC}}\right)$, and the near kinematic distance from the Sun $(d)$. The distances are derived by following Reid et al. (2009) as described in Sect. 4.1.1. Because IRDCs are seen as dark extinction features against the bright Galactic MIR background, it is expected that these clouds lie at the near kinematic distance. In the last column we give the comments concerning the source appearance in the Spitzer 8 and $24-\mu \mathrm{m}$ images. ${ }^{(a)}$ No $24-\mu \mathrm{m}$ image available.

a very poor baseline. The resulting $1 \sigma$ rms noise levels are $40-$ $60 \mathrm{mK}$ at the smoothed resolutions. The final velocity resolution of the smoothed spectra is $0.16 \mathrm{~km} \mathrm{~s}^{-1}$, except for the spectrum towards J18364 SMM1, where it is $0.32 \mathrm{~km} \mathrm{~s}^{-1}$.

The $J=2-1$ transition of $\mathrm{C}^{17} \mathrm{O}$ contains nine hyperfine (hf) components. We fitted the hf structure of the transition using "method hfs" of CLASS90 to derive the LSR velocity $\left(v_{\mathrm{LSR}}\right)$ of the emission, and FWHM linewidth $(\Delta v)$. The hf-line fitting can also be used to derive the line optical thickness, $\tau$. However, in all spectra the hf components are blended and thus the optical thickness cannot be reliably determined. For the rest frequencies of the hf components we used the values from Ladd et al. (1998, Table 6). The adopted central frequency, $224714.199 \mathrm{MHz}$, is that of the $J_{F}=2_{9 / 2} \rightarrow 1_{7 / 2}$ hf component, which has a relative intensity of $R_{i}=\frac{1}{3}$.

\subsection{2. $\mathrm{H}^{13} \mathrm{CO}^{+}(3-2)$ and $\mathrm{DCO}^{+}(3-2)$}

The $\mathrm{H}^{13} \mathrm{CO}^{+}(3-2)$ and $\mathrm{DCO}^{+}(3-2)$ observations were carried out on 9-11 April and 3 June 2010. As frontend we used SHFI/APEX-1 and the backend was the FFTS with a $1 \mathrm{GHz}$ bandwidth divided into 8192 channels. The observations were performed in the WS mode with similar settings as for the $\mathrm{C}^{17} \mathrm{O}(2-1)$ observations. The telescope pointing and focusing were checked by observing the planets Saturn and Jupiter and the pointing was again found to be accurate to $\sim 3^{\prime \prime}$.

The individual spectra were averaged, smoothed, and a first- or third-order polynomial was applied to correct the baseline. The resulting $1 \sigma \mathrm{rms}$ noise values are $20-80 \mathrm{mK}$ for $\mathrm{H}^{13} \mathrm{CO}^{+}(3-2)$ and $30-40 \mathrm{mK}$ for $\mathrm{DCO}^{+}(3-2)$. The final velocity resolutions of the smoothed $\mathrm{H}^{13} \mathrm{CO}^{+}(3-2)$ and $\mathrm{DCO}^{+}(3-2)$ spectra are 0.28 and $0.34 \mathrm{~km} \mathrm{~s}^{-1}$, respectively. As shown in the last column of Table 2 , the on-source integration times were very different between different sources. This is particularly the case for $\mathrm{H}^{13} \mathrm{CO}^{+}(3-2)$, where $t_{\text {int }}$ varies by a factor of $\sim 11$. This explains the considerable variation (factor of 4 ) in the noise values of this transition.

The spectral-line transitions of $\mathrm{H}^{13} \mathrm{CO}^{+}$and $\mathrm{DCO}^{+}$ also have an hf structure (e.g., Schmid-Burgk et al. 2004; Caselli \& Dore 2005). The $J=3-2$ transition we observed is split up into eight and five hf components in the case of $\mathrm{H}^{13} \mathrm{CO}^{+}$and $\mathrm{DCO}^{+}$, respectively. To fit the hf structure, we used the rest frequencies of the $\mathrm{H}^{13} \mathrm{CO}^{+}(3-2)$ and $\mathrm{DCO}^{+}(3-2)$ lines from the Cologne Database for Molecular Spectroscpy (CDMS; Müller et al. 2005) ${ }^{4}$. The adopted central frequency of $\mathrm{H}^{13} \mathrm{CO}^{+}(3-2), 260255.35250 \mathrm{MHz}$, is that of the $J_{F_{1}, F}=$ $3_{7 / 2,4} \rightarrow 25 / 2,3 \mathrm{hf}$ component, which has a relative intensity of $R_{i} \simeq 0.322$. We note that this frequency is $13.5 \mathrm{kHz}$ higher than the value determined by Schmid-Burgk et al. (2004; Table 3$)$. In the case of $\mathrm{DCO}^{+}(3-2)$ the central frequency used, $216112.57790 \mathrm{MHz}$, is that of the $J_{F}=3_{4} \rightarrow 3_{3} \mathrm{hf}$ component; its relative intensity is $R_{i}=\frac{3}{7}$. Caselli \& Dore (2005; their Table 5) determined a $2.1 \mathrm{kHz}$ higher frequency for $\mathrm{DCO}^{+}(3-2)$. The frequency intervals of the hf components for $\mathrm{H}^{13} \mathrm{CO}^{+}(3-2)$ and $\mathrm{DCO}^{+}(3-2)$ are very small. Therefore, the lines overlap significantly, which causes the hf structure to be heavily blended.

\subsection{3. $\mathrm{N}_{2} \mathrm{H}^{+}(3-2)$ and $\mathrm{N}_{2} \mathrm{D}^{+}(3-2)$}

The $\mathrm{N}_{2} \mathrm{H}^{+}(3-2)$ observations towards three of our target positions (I18102 MM1, G015.05 MM1, and G015.31 MM3) were carried out on 29 April 2009. The $\mathrm{N}_{2} \mathrm{D}^{+}(3-2)$ observations were performed during 16-17 and 19-20 October 2008, 26 and 28 April 2009, and 7 June 2010. As frontend we used SHFI/APEX2 and APEX-1 for $\mathrm{N}_{2} \mathrm{H}^{+}(3-2)$ and $\mathrm{N}_{2} \mathrm{D}^{+}(3-2)$, respectively. The backend was the $1 \mathrm{GHz}$ FFTS with 8192 channels. The observations were performed in the WS mode with the similar settings as described above. The pointing and focusing were made by continuum scans on the planets Jupiter, Mars, and Neptune. The pointing was found to be accurate to $\sim 3^{\prime \prime}-5^{\prime \prime}$.

The data were reduced as described above, and a first- or third-order polynomial baseline correction resulted in $1 \sigma \mathrm{rms}$ noise values of $40-100 \mathrm{mK}$ and $20-50 \mathrm{mK}$ in the case of $\mathrm{N}_{2} \mathrm{H}^{+}$ and $\mathrm{N}_{2} \mathrm{D}^{+}$, respectively. The final velocity resolutions of the smoothed $\mathrm{N}_{2} \mathrm{H}^{+}$and $\mathrm{N}_{2} \mathrm{D}^{+}$spectra are 0.26 and $0.32 \mathrm{~km} \mathrm{~s}^{-1}$, respectively. Again, the on-source integration times were very different for different sources, particularly for $\mathrm{N}_{2} \mathrm{D}^{+}(3-2)$ (see Col. 10 of Table 2).

The $J=3-2$ transitions of both $\mathrm{N}_{2} \mathrm{H}^{+}$and $\mathrm{N}_{2} \mathrm{D}^{+}$contain $38 \mathrm{hf}$ components. The hf lines were fitted using the rest frequencies from Pagani et al. (2009b; Tables 4 and 10). The adopted central frequencies of $\mathrm{N}_{2} \mathrm{H}^{+}(3-2)$ and $\mathrm{N}_{2} \mathrm{D}^{+}(3-2), 279511.832$ and $231321.912 \mathrm{MHz}$, are those of the $J_{F_{1} F}=3_{45} \rightarrow 234 \mathrm{hf}$

${ }^{4}$ http://www.astro.uni-koeln.de/cdms/catalog 
Table 2. Observed spectral-line transitions and observational parameters.

\begin{tabular}{lccccccccc}
\hline \hline Transition & $\begin{array}{c}v \\
{[\mathrm{MHz}]}\end{array}$ & $\begin{array}{c}E_{\mathrm{u}} / k_{\mathrm{B}} \\
{[\mathrm{K}]}\end{array}$ & $\begin{array}{c}n_{\text {crit }} \\
{\left[10^{6} \mathrm{~cm}^{-3}\right]}\end{array}$ & $\begin{array}{c}\text { HPBW } \\
{\left[{ }^{\prime \prime}\right]}\end{array}$ & $\begin{array}{l}\eta_{\mathrm{MB}} \\
T_{\text {sys }} \\
{[\mathrm{K}]}\end{array}$ & $\begin{array}{c}\text { Channel spacing } \\
{[\mathrm{kHz}]}\end{array}$ & $\begin{array}{c}t_{\text {int }} \\
{\left[\mathrm{km} \mathrm{s}^{-1}\right]}\end{array}$ & {$[\mathrm{min}]$} \\
\hline $\mathrm{DCO}^{+}(3-2)$ & $216112.57790^{b}$ & 20.7 & 1.8 & 28.9 & 0.75 & $150-184$ & 122.07 & 0.17 & $5.5-13.5$ \\
$\mathrm{C}^{17} \mathrm{O}(2-1)$ & $224714.199^{c}$ & 16.2 & 0.01 & 27.8 & 0.75 & $163-218$ & $61.04^{d}$ & $0.08^{d}$ & $5-7.5$ \\
$\mathrm{~N}_{2} \mathrm{D}^{+}(3-2)$ & $231321.912^{e}$ & 22.2 & 1.7 & 27.0 & 0.75 & $172-239$ & 122.07 & 0.16 & $6.5-48$ \\
$\mathrm{H}^{13} \mathrm{CO}^{+}(3-2)$ & $260255.35250^{b}$ & 25.0 & 3.1 & 24.0 & 0.74 & $232-243$ & 122.07 & 0.14 & $2.5-27$ \\
$\mathrm{~N}_{2} \mathrm{H}^{+}(3-2)$ & $279511.832^{e}$ & 26.8 & 3.0 & 22.3 & 0.74 & $165-175$ & 122.07 & 0.13 & $1.5-6.5$ \\
\hline
\end{tabular}

Notes. Columns (2)-(4) give the rest frequencies of the observed transitions $(v)$, their upper state energies $\left(E_{\mathrm{u}} / k_{\mathrm{B}}\right.$, where $k_{\mathrm{B}}$ is the Boltzmann constant), and critical densities. Critical densities were calculated at $T \sim 15 \mathrm{~K}$ (typical value in IRDCs) using the collisional rate data available in the Leiden Atomic and Molecular Database (LAMDA; http://www. strw. leidenuniv.nl/ moldata/) (Schöier et al. 2005). For $\mathrm{N}_{2} \mathrm{D}^{+}$, we used the Einstein $A$-coefficient from Pagani et al. (2009b) and the same collisional rate as for $\mathrm{N}_{2} \mathrm{H}^{+}$. Columns (5)-(10) give the APEX beamsize (HPBW) and the main beam efficiency $\left(\eta_{\mathrm{MB}}\right)$ at the observed frequencies, and the SSB system temperatures during the observations ( $T_{\text {sys }}$ in $T_{\mathrm{A}}^{*}$ scale, see text), channel widths (both in $\mathrm{kHz}$ and $\mathrm{km} \mathrm{s}^{-1}$ ) of the original data, and the on-source integration times per position $\left(t_{\text {int }}\right)$. ${ }^{(a)}$ The original channel spacings. The final spectra were Hanning-smoothed, which divides the number of channels by two. ${ }^{(b)}$ From the CDMS spectroscopic database (Müller et al. 2005). ${ }^{(c)}$ From Ladd et al. (1998). ${ }^{(d)}$ For the $\mathrm{C}^{17} \mathrm{O}(2-1)$ observations towards J18364 SMM1, channel width is $122.07 \mathrm{kHz}$ or $0.16 \mathrm{~km} \mathrm{~s}^{-1}$. (e) From Pagani et al. (2009b).

component, which has a relative intensity of $R_{i}=\frac{11}{63}$. In these cases the hf components are blended as well and consequently the value of $\tau$ cannot be reliably determined through hf fitting.

\subsection{Archival dust continuum data}

Submillimetre and/or millimetre dust continuum maps of our sources are published in the papers by Beuther et al. (2002a; MAMBO $1.2 \mathrm{~mm}$ ), Williams et al. (2004; SCUBA 450 \& $850 \mu \mathrm{m})$, Rathborne et al. (2006; MAMBO-II $1.2 \mathrm{~mm}$ ), and Birkmann et al. (2006; SCUBA $450 \& 850 \mu \mathrm{m})$. For the sources I18102 MM1, I18182 MM2, and J18364 SMM1 we retrieved $850 \mu \mathrm{m}$ dust continuum data from the JCMT/SCUBA archive (Di Francesco et al. 2008) ${ }^{5}$. No archival SCUBA data were available for the rest of our sources. Thus, for the sources G015.05 MM1, G015.31 MM3, and I18223 MM3 we extracted Bolocam 1.1-mm maps from images produced by the Bolocam Galactic Plane Survey (BGPS; see, e.g., Bally et al. 2010, and references therein) ${ }^{6}$. For I18151 MM2, no archival (sub)mm data were found and thus we used the MAMBO 1.2-mm map of this source published in the paper by Beuther et al. (2002a; kindly provided by $\mathrm{H}$. Beuther).

In the present paper the (sub)mm dust continuum data are used in particular to determine the $\mathrm{H}_{2}$ column densities towards the line-observation positions. These are used to derive the fractional abundances of the observed molecules.

\subsection{Spitzer infrared data}

We also used Spitzer IRAC 8- $\mu \mathrm{m}$ and MIPS $24-\mu \mathrm{m}$ images of the sources to investigate the clump associations with protostellar activity. The $8-\mu \mathrm{m}$ images are from the Spitzer Galactic Legacy Infrared Mid-Plane Survey Extraordinaire (GLIMPSE; Benjamin et al. 2003) ${ }^{7}$. The 24- $\mu \mathrm{m}$ images (not shown in the paper) were taken from the Spitzer MIPS GALactic plane survey (MIPSGAL; Carey et al. 2009) ${ }^{8}$. In Fig. 1 we show the Spitzer/IRAC 8- $\mu \mathrm{m}$ images of the clumps overlaid with contours of (sub)mm dust continuum emission. We note that better quality Spitzer data of the sources are available and already published (see references in Appendix B). However, in the present work we

\footnotetext{
5 http://cadcwww.dao.nrc.ca/jcmt/

6 http://irsa.ipac.caltech.edu/data/BOLOCAM_GPS/

7 http://irsa.ipac. caltech.edu/data/SPITZER/GLIMPSE/

${ }^{8}$ http://irsa.ipac.caltech. edu/data/SPITZER/MIPSGAL/
}

did not use Spitzer data to derive the physical properties of the sources; the data are used to illustrate the source appearance at MIR wavelengths.

\section{Observational results}

\subsection{Spectra}

The Hanning-smoothed spectra are shown in Fig. 2. Note that all data are presented in units of $T_{\mathrm{A}}^{*}$. The $\mathrm{C}^{17} \mathrm{O}(2-1)$ line is clearly detected towards all sources, but the hf structure of the line is completely blended in all cases except for G015.31 MM3, where it is partially resolved. The hf structure is not resolved because the linewidths are wider than the separation in velocity of individual hf components.

The $\mathrm{H}^{13} \mathrm{CO}^{+}(3-2)$ line is also clearly detected towards all target positions, but towards G015.31 MM3 the line is very weak (only $\sim 3 \sigma$ ). The "double peak"-like profiles in some of the smoothed $\mathrm{H}^{13} \mathrm{CO}^{+}(3-2)$ spectra can be attributed to the noise in most cases. In the case of J18364 SMM1, the self-absorption dip is a distinctive feature also in the unsmoothed spectrum, and thus likely to be real. $\mathrm{DCO}^{+}(3-2)$ emission is seen towards all sources, but the line is very weak in I18102 MM1 and G015.31 MM3 (detected only at $\sim 3 \sigma$ ).

$\mathrm{N}_{2} \mathrm{H}^{+}(3-2)$ line-observations were performed only towards three of our sources, and the line is quite strong in all cases. The $\mathrm{N}_{2} \mathrm{H}^{+}(3-2)$ line of I18102 MM1 exhibits red asymmetry with a central dip near the velocity of the strongest hf component; this feature is also clearly visible in the unsmoothed spectrum. The $\mathrm{N}_{2} \mathrm{D}^{+}(3-2)$ line is detected towards all sources except G015.31 MM3, I18182 MM2, and J18364 SMM1, where the spectra have a low $\mathrm{S} / \mathrm{N}$ ratio.

\subsubsection{Other line detections}

All observed sources show additional spectral lines in the observed frequency bands, mostly in the spectra covering the $\mathrm{DCO}^{+}(3-2)$ and $\mathrm{N}_{2} \mathrm{D}^{+}(3-2)$ lines. The line identification was made with Weeds, which is an extension of CLASS (Maret et al. 2011), and applying the Jet Propulsion Laboratory ${ }^{9}$ (JPL; Pickett et al. 1998) and CDMS spectroscopic databases. We used the LTE modelling application of Weeds to check if all predicted lines of a candidate molecule were present in the

\footnotetext{
${ }^{9}$ http://spec.jpl.nasa.gov/
} 

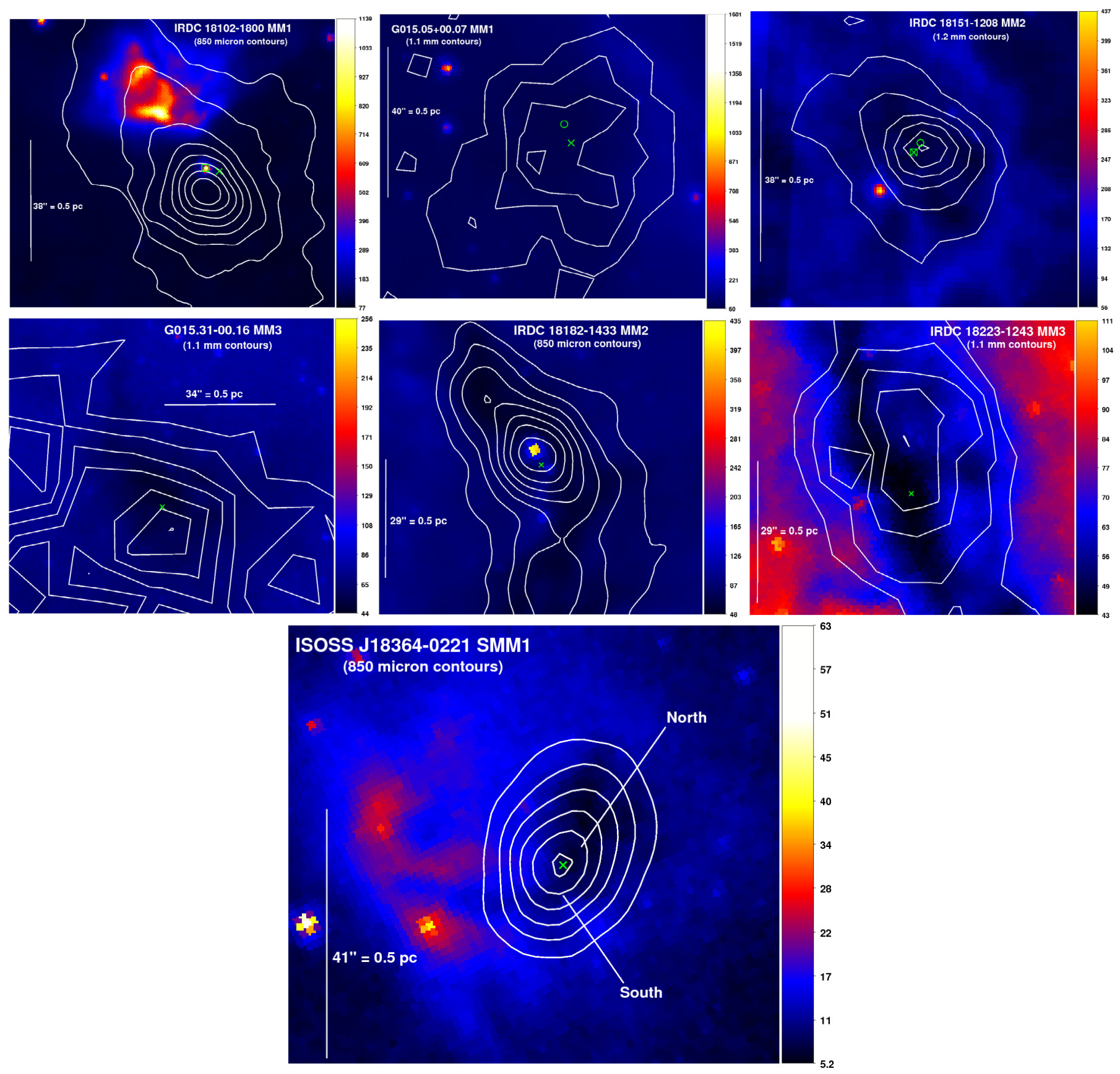

Fig. 1. Spitzer/IRAC 8- $\mu$ m images overlaid with contours of (sub)mm dust continuum emission (SCUBA $850 \mu$ m, Bolocam 1.1 mm, MAMBO $1.2 \mathrm{~mm}$ ). The colour bars indicate the $8-\mu \mathrm{m}$ intensity scales in units of $\mathrm{MJy} \mathrm{sr}^{-1}$. The contour step-size is $0.1 \mathrm{Jy}^{-1}$ beam $^{-1}$ in all cases except

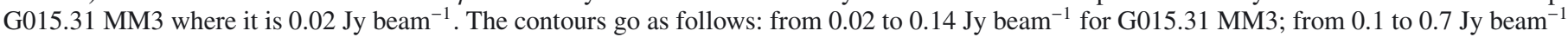
for G015.05 MM1 and I18151 MM2; from 0.2 to $0.7 \mathrm{Jy} \mathrm{beam}^{-1}$ for J18364 SMM1; from 0.2 to $0.9 \mathrm{Jy}_{\text {beam }}{ }^{-1}$ for I18182 MM2; from 0.2 to $2.3 \mathrm{Jy} \mathrm{beam}^{-1}$ for I18102 MM1; and from 0.3 to $0.7 \mathrm{Jy} \mathrm{beam}^{-1}$ for I18223 MM3. The crosses indicate the positions of our molecular-line observations. The box symbol towards I18102 MM1 indicates the position of the $6.7 \mathrm{GHz}$ Class II CH $\mathrm{CH}_{3} \mathrm{OH}$ maser (Beuther et al. 2002b). For I18151 MM2, the box symbol shows the position of the $84.5 \mathrm{GHz}$ Class I $\mathrm{CH}_{3} \mathrm{OH}$ maser (Marseille et al. 2010b); it coincides with our target position. The circles indicate the positions of the $22.2 \mathrm{GHz} \mathrm{H}_{2} \mathrm{O}$ masers associated with I18151 MM2 (Beuther et al. 2002b) and G015.05 MM1 (Wang et al. 2006). The mm peak positions of the northern and southern cores within the clump J18364 SMM1 are indicated (Hennemann et al. 2009). The scale bar in each panel corresponds to $0.5 \mathrm{pc}$.

observed spectrum (see Sect. 4.2). In some cases, we were able to reject some line candidates on the basis of non-detection of other transitions expected at nearby frequencies.

I18102 MM1 and I18151 MM2 show relatively line-rich spectra at $\sim 231 \mathrm{GHz}$ and $\sim 216 \mathrm{GHz}$, in the USB and LSB, respectively. Two additional lines at $\sim 231 \mathrm{GHz}$ (USB) are also visible in the spectrum of I18182 MM2 (see Fig. 3). The only unambiguous line identification in the spectrum of I18102 MM1 is ${ }^{13} \mathrm{CS}(5-4)$. The second-strongest line $\left(T_{\mathrm{A}}^{*}=0.12 \mathrm{~K}\right)$ can be assigned to $\mathrm{C}^{18} \mathrm{O}(2-1)$ at $\sim 219.56 \mathrm{GHz}$ seen in the image side band (marked with "image"). The strongest line in the spectrum $\left(T_{\mathrm{A}}^{*}=0.13 \mathrm{~K}\right)$ might be caused by $\mathrm{CNCHO}\left(J_{K_{a}, K_{c}}=\right.$ $\left.23_{0,23}-22_{0,22}\right)$ at $\sim 219.53 \mathrm{GHz}$ arising from the image band, or it could represent an additional $\mathrm{C}^{18} \mathrm{O}(2-1)$ velocity component 


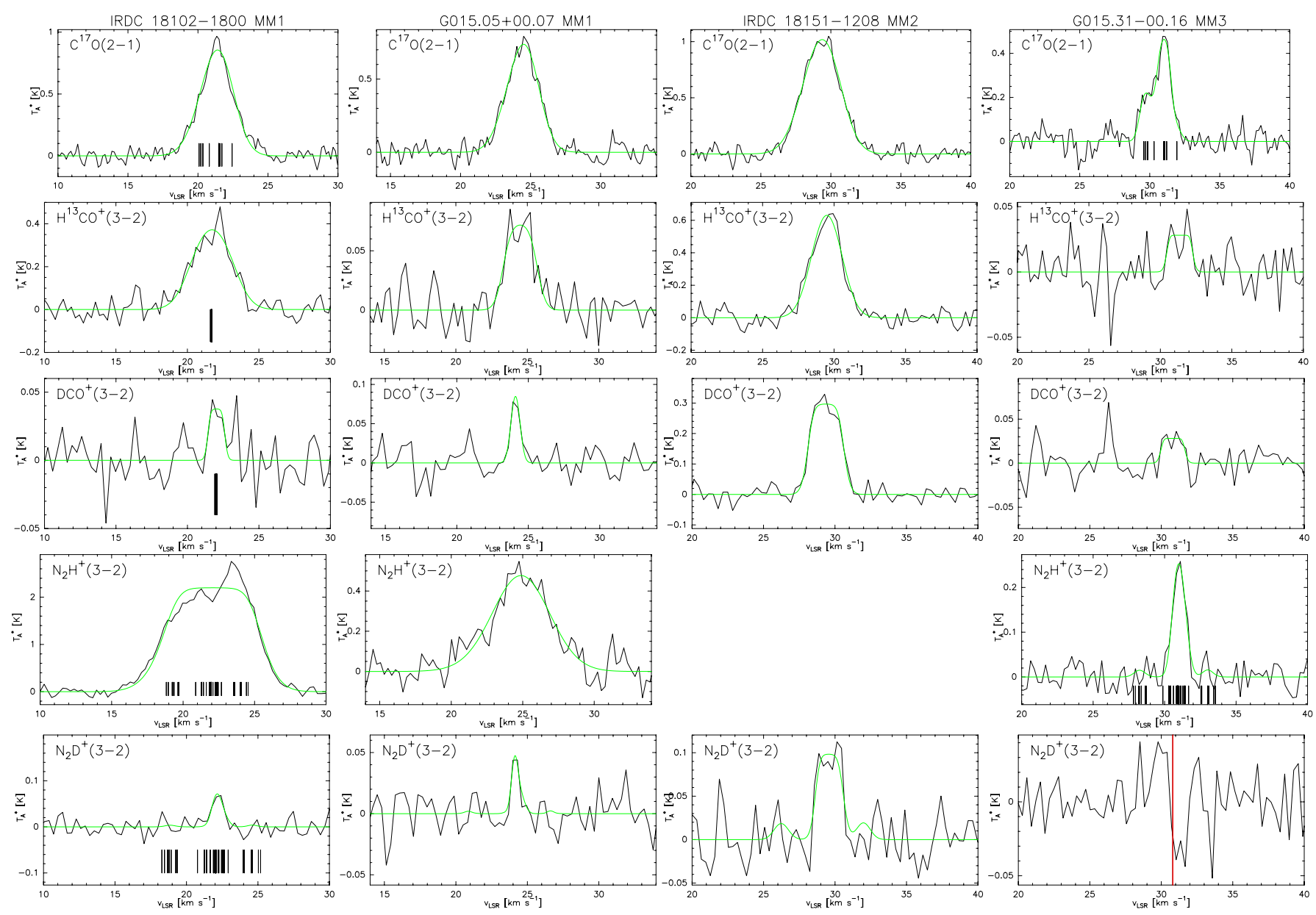

Fig. 2. Smoothed $\mathrm{C}^{17} \mathrm{O}(2-1), \mathrm{H}^{13} \mathrm{CO}^{+}(3-2), \mathrm{DCO}^{+}(3-2), \mathrm{N}_{2} \mathrm{H}^{+}(3-2)$, and $\mathrm{N}_{2} \mathrm{D}^{+}(3-2)$ spectra (top to bottom) towards the clumps (left to right). Overlaid on the spectra are the hf-structure fits. The relative velocities of each individual hf components in each observed transition are labelled with a short bar on the spectra towards I18102 MM1; these are also shown in the $\mathrm{C}^{17} \mathrm{O}$ and $\mathrm{N}_{2} \mathrm{H}^{+}$spectra towards G015.31 MM3. The red vertical line on the $\mathrm{N}_{2} \mathrm{D}^{+}$spectrum towards G015.31 MM3 shows the $v_{\text {LSR }}=30.81 \mathrm{~km} \mathrm{~s}^{-1}$ of the clump as measured from $\mathrm{N}_{2} \mathrm{H}^{+}(1-0)$ by SSK08. Note that $\mathrm{N}_{2} \mathrm{H}^{+}(3-2)$ observations were carried out only towards three sources and that $\mathrm{N}_{2} \mathrm{D}^{+}(3-2)$ was not detected towards G015.31 MM3, I18182 MM2, and J18364 SMM1.

along the line of sight. The latter is likely to be true because of the relatively strong line intensity. The line at $\sim 231.06 \mathrm{GHz}$ can be assigned to either OCS(19-18) or $\mathrm{CH}_{3} \mathrm{NH}_{2}-\mathrm{E}\left(J_{K_{a}, K_{c}}=\right.$ $7_{2,5}-7_{1,5}$ ) because these two transitions are blended. However, it could also be caused by yet another velocity component of $\mathrm{C}^{18} \mathrm{O}(2-1)$ seen towards I18102 MM1. From the spectrum towards I18151 MM2 we identified the $\mathrm{C}_{2} \mathrm{D}(N=3-2)$ line separated into two hf groups. This transition is split into $13 \mathrm{hf}$ components, of which the detected two groups contain 10 components together. The hf group at $\sim 216.43 \mathrm{GHz}$ is blended with $\mathrm{CH}_{2} \mathrm{CHC}^{15} \mathrm{~N}\left(J_{K_{a}, K_{c}}=25_{2,24}-25_{0,25}\right)$. The $J_{K_{a}, K_{c}}=3_{3,0}-2_{2,1}$ transition of ortho-c- $\mathrm{C}_{3} \mathrm{H}_{2}$ is seen towards I18151 MM2, but the line at $\sim 216.45 \mathrm{GHz}$ is unidentified (marked with " $U$ "). The $\mathrm{CH}_{3} \mathrm{COCH}_{3}$-EA $\left(J_{K_{a}, K_{c}}=15_{9,7}-14_{8,6}\right)$ line in the USB is possibly detected towards I18182 MM2. However, it is blended with $\mathrm{C}^{18} \mathrm{O}(2-1)$ entering via the image band. The relatively strong intensity of $T_{\mathrm{A}}^{*}=0.17 \mathrm{~K}$ suggests the line is caused by $\mathrm{C}^{18} \mathrm{O}(2-1)$. The weaker nearby line could be related to an additional $\mathrm{C}^{18} \mathrm{O}(2-1)$ velocity component.

The $J_{K_{a}, K_{c}}=3_{3,0}-2_{2,1}$ transition of ortho-c- $\mathrm{C}_{3} \mathrm{H}_{2}$ at $\sim 216.3 \mathrm{GHz}$ in the LSB is also detected towards I18102 MM1, I18182 MM2, and I18223 MM3 (Fig. 4). A thermal $(v=0)$ $\mathrm{SiO}(6-5)$ line at $\sim 260.5 \mathrm{GHz}$ in the USB is seen towards 118102 MM1, I18151 MM2, and I18223 MM3 (Fig. 5). In particular, the
$\mathrm{SiO}(6-5)$ line of I18151 MM2 exhibits very broad wing emission. Finally, as in the cases of I18102 MM1 and I18182 MM2, we detected the $J=2-1$ transition of $\mathrm{C}^{18} \mathrm{O}$ at $\sim 219.56 \mathrm{GHz}$ in the image side band towards G015.05 MM1, I18151 MM2, G015.31 MM3, I18223 MM3, and J18364 SMM1 (Fig. 6). The intensities of these $\mathrm{C}^{18} \mathrm{O}$ lines, $T_{\mathrm{A}}^{*} \sim 0.09-0.31 \mathrm{~K}$, are mostly comparable to those possibly detected towards I18102 MM1 and I18182 MM2. Note that the lines "leaking" from the rejected image sideband are heavily attenuated by the sideband filter. Thus, we cannot establish the correct intensity scale for the imageband lines.

In Table 3 we list the observed extra transitions, their rest frequencies (observed frequency for the U-line), and upperstate energies. The rest frequencies and upper-state energies were assigned using the JPL, CDMS, and Splatalogue ${ }^{10}$ (Remijan et al. 2007) spectroscopic databases. The additional lines/species are discussed in more detail in Appendix A.

\subsection{Spectral line parameters}

The spectral line parameters are given in Table 4. In this table we give the radial velocity $\left(v_{\mathrm{LSR}}\right)$, FWHM linewidth $(\Delta v)$, peak

${ }^{10}$ http://www.splatalogue.net/ 
O. Miettinen et al.: Massive clumps in IRDCs

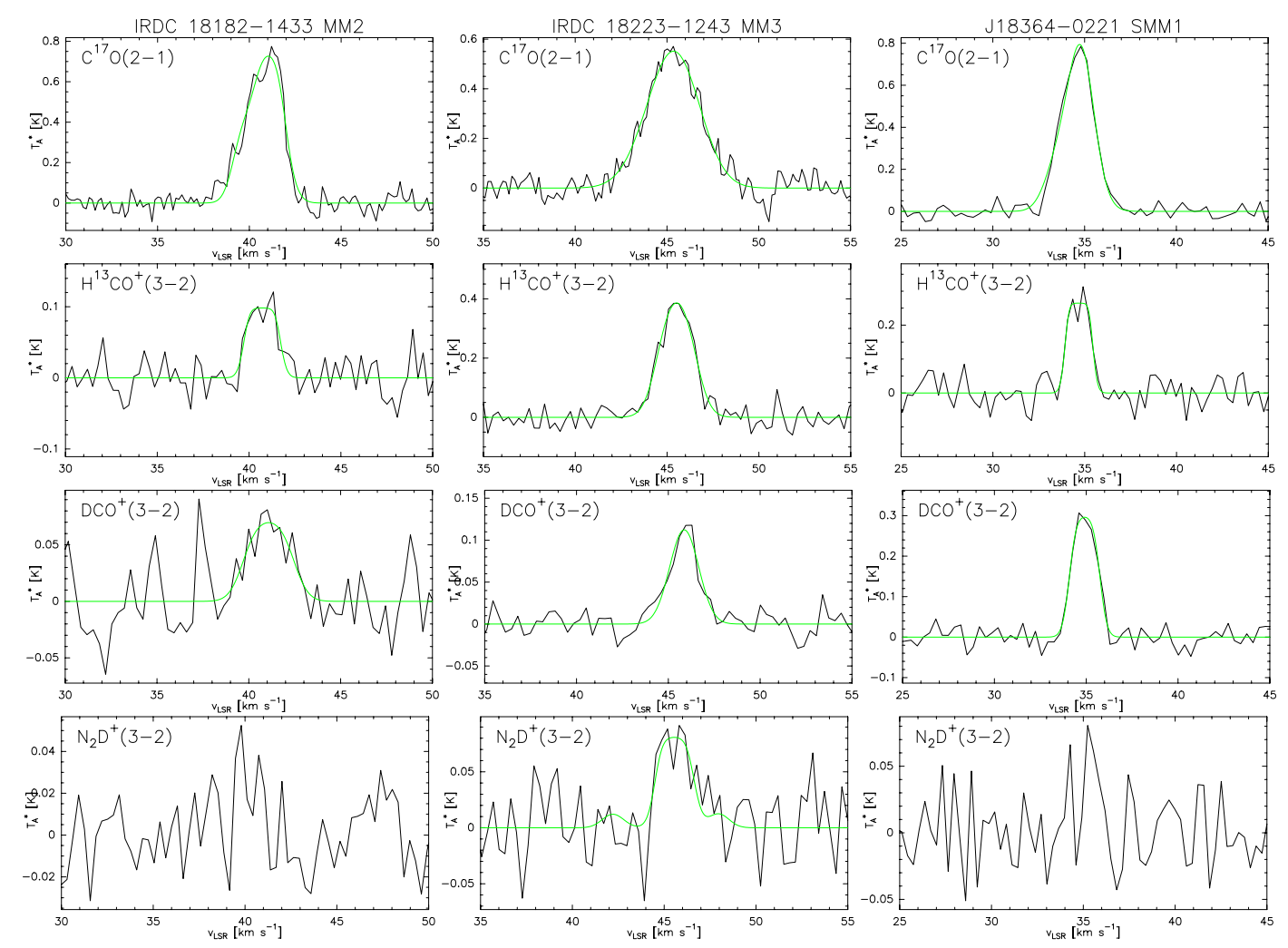

Fig. 2. continued.

intensity $\left(T_{\mathrm{A}}^{*}\right)$, integrated intensity $\left(\int T_{\mathrm{A}}^{*} \mathrm{~d} v\right)$, peak optical depth of the line $\left(\tau_{0}\right)$, and excitation temperature $\left(T_{\text {ex }}\right)$. The values of $v_{\mathrm{LSR}}$ and $\Delta v$ for $\mathrm{C}^{17} \mathrm{O}, \mathrm{H}^{13} \mathrm{CO}^{+}, \mathrm{DCO}^{+}, \mathrm{N}_{2} \mathrm{H}^{+}, \mathrm{N}_{2} \mathrm{D}^{+}$, and $\mathrm{C}_{2} \mathrm{D}$ were derived through fitting the hf structure. For the other lines these parameters were derived by fitting a single Gaussian to the line profile. The values of $T_{\mathrm{A}}^{*}$ were also determined by fitting the lines with a single Gaussian. The velocity-integrated intensities $\int T_{\mathrm{A}}^{*} \mathrm{~d} v$ were calculated over the velocity range given in Col. (6) of Table 4. The uncertainties reported in $v_{\mathrm{LSR}}, \Delta v, T_{\mathrm{A}}^{*}$, and $\int T_{\mathrm{A}}^{*} \mathrm{~d} v$ represent the formal $1 \sigma$ errors determined by the fitting routine. We used RADEX ${ }^{11}$ (van der Tak et al. 2007) to determine the values of $\tau_{0}$ and $T_{\text {ex }}$ for the lines of $\mathrm{C}^{17} \mathrm{O}, \mathrm{H}^{13} \mathrm{CO}^{+}$, $\mathrm{DCO}^{+}, \mathrm{N}_{2} \mathrm{H}^{+}, \mathrm{c}-\mathrm{C}_{3} \mathrm{H}_{2}, \mathrm{OCS},{ }^{13} \mathrm{CS}$, and SiO. RADEX modelling is described in more detail in Sect. 4.2, where we discuss the determination of molecular column densities. For $\mathrm{N}_{2} \mathrm{D}^{+}(3-2)$ we used as $T_{\text {ex }}$ the value derived for $\mathrm{N}_{2} \mathrm{H}^{+}(3-2)$ or, when not possible, we adopted $T_{\mathrm{ex}}\left[\mathrm{N}_{2} \mathrm{H}^{+}(1-0)\right]$ from SSK08. We note that the $T_{\text {ex }}$ values obtained for the $J=3-2$ transition of $\mathrm{N}_{2} \mathrm{H}^{+}$are comparable to those of $J=1-0$ from SSK08.

\section{Analysis and results}

\subsection{Revision of clump properties}

In this subsection, we present recalculations of several clump properties presented previously in the literature (see Tables 1 and 5).

\subsubsection{Kinematic distances}

Sakai et al. (2008) calculated the near kinematic distance for all of our clumps except J18364 SMM1 (see their Table 4). They

11 http://www.strw.leidenuniv.nl/ moldata/radex.html used the LSR velocity of $\mathrm{N}_{2} \mathrm{H}^{+}(1-0)$ and the rotation curve of Clemens (1985) with the standard rotation parameters $\left(\Theta_{0}\right.$, $\left.R_{0}\right)=\left(220 \mathrm{~km} \mathrm{~s}^{-1}, 8.5 \mathrm{kpc}\right)$, where $\Theta_{0}$ is the circular orbital speed of the Sun, and $R_{0}$ is the galactocentric distance of the Sun. Because $\mathrm{N}_{2} \mathrm{H}^{+}$is a tracer of high-density gas, the radial velocity derived from $\mathrm{N}_{2} \mathrm{H}^{+}$lines is suitable to determine the source kinematic distance. The hf structure of $\mathrm{N}_{2} \mathrm{H}^{+}(1-0)$ was also resolved (to some degree) towards many of the sources by SSK08, and thus the hf-structure fitting is expected to yield a reliable centroid velocity. Towards the three sources for which we detected $\mathrm{N}_{2} \mathrm{H}^{+}(3-2)$ lines, the obtained values of $v_{\mathrm{LSR}}$ are comparable to those from $\mathrm{N}_{2} \mathrm{H}^{+}(1-0)$. We recalculated the distances from SSK08 using the recent rotation curve of Reid et al. (2009), which is based on measurements of trigonometric parallaxes and proper motions of masers in high-mass star-forming regions. The best-fit rotation parameters of Reid et al. (2009) are $\left(\Theta_{0}, R_{0}\right)=\left(254 \mathrm{~km} \mathrm{~s}^{-1}, 8.4 \mathrm{kpc}\right)$. The resulting galactocentric distances and near kinematic distances $\left(R_{\mathrm{GC}}\right.$ and $\left.d\right)$ are given in Cols. (6) and (7) of Table 1. The revised distances differ at most by $0.2 \mathrm{kpc}$ from those reported by SSK08.

The source J18364 SMM1 is an exception. Its CO(1-0) radial velocity of $\sim 33 \mathrm{~km} \mathrm{~s}^{-1}$ corresponds to a kinematic distance of $\sim 2.2 \mathrm{kpc}$ according to the Brand \& Blitz (1993) rotation curve (see Birkmann et al. 2006). We recomputed this distance from the LSR velocity of $\mathrm{C}^{17} \mathrm{O}(2-1)\left(34.8 \mathrm{~km} \mathrm{~s}^{-1}\right)$ because it is tracing higher density gas than the main $\mathrm{CO}$ isotopologue (similar radial velocity was obtained for the other detected transitions). The revised distance is $\sim 2.5 \mathrm{kpc}$ according to the Reid et al. (2009) rotation curve; the previous value of $2.2 \mathrm{kpc}$ is within the errors.

We note that for the sources I18102 MM1, I18151 MM2, I18182 MM2, and I18223 MM3 there are kinematic distance estimates $(2.6,3.0,4.5$, and $3.7 \mathrm{kpc}$, respectively) based on 


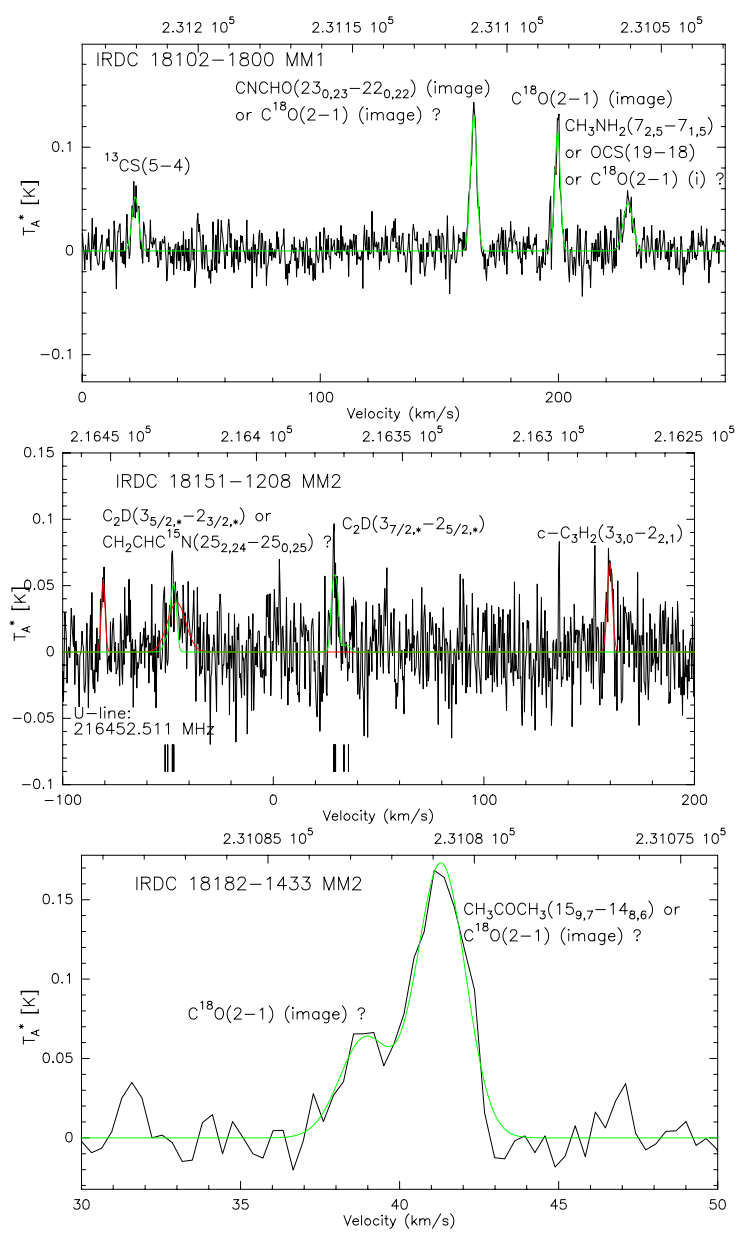

Fig. 3. Multi-line spectra of I18102 MM1, I18151 MM2, and I18182 MM2. On the spectrum towards I18151 MM2, the green line shows the hf-structure fit to $\mathrm{C}_{2} \mathrm{D}(3-2)$, and the red line indicates single Gaussian fits to the other lines. The relative velocities of the $C_{2} D(3-2)$ hf components are labelled with a short bar. On the other spectra, the green line represents single Gaussian fits.

the $\mathrm{CS}(2-1)$ velocity and the rotation curve of Brand \& Blitz (1993) (see Sridharan et al. 2002). For both G015.05 MM1 and G015.31 MM3, Rathborne et al. (2006) used the kinematic distance of $3.2 \mathrm{kpc}$ derived from the ${ }^{13} \mathrm{CO}(1-0)$ velocity and the rotation curve of Clemens (1985). Our distances differ by $0.1-$ $1.0 \mathrm{kpc}$ from the above values.

\subsubsection{Gas and dust temperatures}

Sridharan et al. (2005) determined the $\mathrm{NH}_{3}$ rotational temperature, $T_{\text {rot }}\left(\mathrm{NH}_{3}\right)$, for three of our sources (I18151 MM2, I18182 MM2, and I18223 MM3) at the 40" angular resolution. Sakai et al. (2008) have determined $T_{\text {rot }}\left(\mathrm{NH}_{3}\right)$ for all of our sources except J18364 SMM1 (see their Table 10). The values of $T_{\text {rot }}\left(\mathrm{NH}_{3}\right)$ from the above two studies are otherwise similar except in the case of I18223 MM3. Sridharan et al. reported the value $32.7 \mathrm{~K}$ for this source, whereas SSK08 obtained a temperature lower by a factor of two $\left(16.2_{-0.9}^{+1.0} \mathrm{~K}\right)$ at an angular resolution about 1.8 times poorer (73"). The $T_{\text {rot }}\left(\mathrm{NH}_{3}\right)$ value for J18364 SMM1 at the $40^{\prime \prime}$ resolution is $10.75 \mathrm{~K}$ (Krause 2003). We converted $T_{\text {rot }}\left(\mathrm{NH}_{3}\right)$ from Krause (2003) and SSK08 into the gas kinetic temperature, $T_{\text {kin }}$, using the relationship given by Tafalla et al. (2004, Appendix B). The uncertainty in $T_{\text {kin }}$ was calculated by propagating the larger of the two $T_{\text {rot }}$-errors given by SSK08.
Table 3. Other candidate species/transitions observed.

\begin{tabular}{lcc}
\hline \hline $\begin{array}{l}\text { Species/ } \\
\text { transition }\end{array}$ & $\begin{array}{c}v \\
{[\mathrm{MHz}]}\end{array}$ & $\begin{array}{c}E_{\mathrm{u}} / k_{\mathrm{B}} \\
{[\mathrm{K}]}\end{array}$ \\
\hline ortho-c-C $_{3} \mathrm{H}_{2}\left(J_{K_{a}, K_{c}}=3_{3,0}-2_{2,1}\right) v=0$ & $216278.7560(\mathrm{JPL})$ & 19.5 \\
$\mathrm{C}_{2} \mathrm{D}\left(N_{J, F}=3_{7 / 2,9 / 2}-25 / 2,7 / 2\right)^{b}$ & 216372.8300 (CDMS) & 20.8 \\
$\mathrm{CH}_{2} \mathrm{CHC}{ }^{15} \mathrm{~N}\left(J_{K_{a}, K_{c}}=25_{2,24}-25_{0,25}\right)^{c}$ & $216428.6683(\mathrm{JPL})$ & 151.8 \\
$\mathrm{U}$ & 216452.5110 & $\ldots$ \\
$\mathrm{CNCHO}\left(J_{K_{a}, K_{c}}=23_{0,23}-22_{0,22}\right)^{d}$ & $219531.6000(\mathrm{CDMS})$ & 127.2 \\
$\mathrm{C}^{18} \mathrm{O}(J=2-1)^{d}$ & $219560.3600(\mathrm{JPL})$ & 15.8 \\
$\mathrm{CH}_{3} \mathrm{NH}_{2}-\mathrm{E}\left(J_{K_{a}, K_{c}}=7_{2,5}-7_{1,5}\right)^{e}$ & $231060.6041(\mathrm{JPL})$ & 75.6 \\
$\mathrm{OCS}(J=19-18)$ & $231060.9830(\mathrm{JPL})$ & 110.9 \\
$\mathrm{CH}_{3} \mathrm{COCH}{ }_{3}-\mathrm{EA}\left(J_{K_{a}, K_{c}}=15_{9,7}-14_{8,6}\right) v=0$ & $231080.9647(\mathrm{JPL})$ & 95.9 \\
${ }^{13} \mathrm{CS}(J=5-4)$ & $231220.9960(\mathrm{JPL})$ & 33.3 \\
$\mathrm{SiO}(J=6-5)$ & $260518.0200(\mathrm{JPL})$ & 43.8 \\
\hline
\end{tabular}

Notes. ${ }^{(a)}$ For asymmetric top molecules, $K_{a}$ and $K_{c}$ refer to the projection of the angular momentum along the $a$ and $c$ principal axes. The $\mathrm{CH}_{2} \mathrm{CHC}^{15} \mathrm{~N}$ and $\mathrm{CNCHO}$ transitions are of type $a\left(\Delta K_{a}=0, \pm 2, \ldots\right.$ and $\left.\Delta K_{c}= \pm 1, \pm 3, \ldots\right)$, the $o-\mathrm{c}-\mathrm{C}_{3} \mathrm{H}_{2}$ and $\mathrm{CH}_{3} \mathrm{COCH}_{3}$-EA transitions are $b$-type $\left(\Delta K_{a}=\Delta K_{c}= \pm 1, \pm 3, \ldots\right)$, and the $\mathrm{CH}_{3} \mathrm{NH}_{2}$-E transition exbits $c$-type selection rules $\left(\Delta K_{a}= \pm 1, \pm 3, \ldots\right.$ and $\Delta K_{c}=$ $0, \pm 2, \ldots$ ) (Gordy \& Cook 1984). ${ }^{(b)}$ This is the strongest hf component and it is blended with the $J, F=7 / 2,5 / 2-5 / 2,3 / 2$ and $J, F=$ $7 / 2,7 / 2-5 / 2,5 / 2$ hf components. ${ }^{(c)}$ Blended with the $\mathrm{C}_{2} \mathrm{D}(3-2) \mathrm{hf}$ group. ${ }^{(d)}$ Seen in the image band. The candidate lines of CNCHO, $\mathrm{CH}_{3} \mathrm{NH}_{2}$-E, OCS, and $\mathrm{CH}_{3} \mathrm{COCH}_{3}$-EA are possibly blended with $\mathrm{C}^{18} \mathrm{O}(2-1)$ coming from the image band. ${ }^{(e)}$ The corresponding vibrational level is $E_{1}, l=-1$. This line is blended with OCS(19-18).

For three of our sources, G015.05 MM1, I18223 MM3, and J18364 SMM1, dust temperature estimates have been made by Rathborne et al. (2010), Beuther et al. (2010), and Birkmann et al. (2006), respectively. The determined dust temperature values of G015.05 MM1, 11.0-36.0 K, bracket the $T_{\text {kin }}$ value of 17.2 K. A dust temperature of I18223 MM3 was also derived by Beuther \& Steinacker (2007) from the source SED using the Spitzer/MIPS data at 24 and $70 \mu \mathrm{m}$, and MAMBO $1.2 \mathrm{~mm}$ and PdBI $3.2 \mathrm{~mm}$ data. The value $15 \mathrm{~K}$ they obtained for the cold part of the spectrum is three $\mathrm{K}$ lower than the value $18 \mathrm{~K}$ derived recently by Beuther et al. (2010). The latter also used the Herschel/PACS (70, 100, and $160 \mu \mathrm{m})$ and SPIRE (250, 350, and $500 \mu \mathrm{m})$ data, and SCUBA $850 \mu \mathrm{m}$ data to construct the source SED (but not the 3.2-mm flux density). The dust temperature of I18223 MM3 is comparable to its gas temperature of 18.7 $\mathrm{K}$. By utilising the far-infrared and submm flux density ratios, Birkmann et al. (2006) deduced the value $T_{\text {dust }}=16.5_{-3.0}^{+6.0} \mathrm{~K}$ for J18364 SMM1, which is higher than the gas temperature $11.4 \mathrm{~K}$.

The gas and dust temperatures of the clumps are given in Cols. (2) and (3) of Table 5.

\subsubsection{Clump masses, radii, and $\mathrm{H}_{2}$ column and number densities}

We calculated the masses and beam-averaged $\mathrm{H}_{2}$ column densities of the clumps using the formulas

$$
\begin{aligned}
& M=\frac{S_{v} d^{2}}{B_{v}\left(T_{\text {dust }}\right) \kappa_{v} R_{\mathrm{d}}}, \\
& N\left(\mathrm{H}_{2}\right)=\frac{I_{v}^{\text {dust }}}{B_{v}\left(T_{\text {dust }}\right) \mu_{\mathrm{H}_{2}} m_{\mathrm{H}} \kappa_{v} R_{\mathrm{d}}} .
\end{aligned}
$$

In the above formulas, $I_{v}^{\text {dust }}$ and $S_{v}$ are the peak surface brightness and integrated flux density of the (sub)mm dust emission; note that $I_{v}^{\text {dust }}=S_{v}^{\text {peak }} / \Omega_{\text {beam }}$, where $S_{v}^{\text {peak }}$ is the peak flux density and $\Omega_{\text {beam }}$ is the solid angle of the telescope beam. $B_{v}\left(T_{\text {dust }}\right)$ 
O. Miettinen et al.: Massive clumps in IRDCs

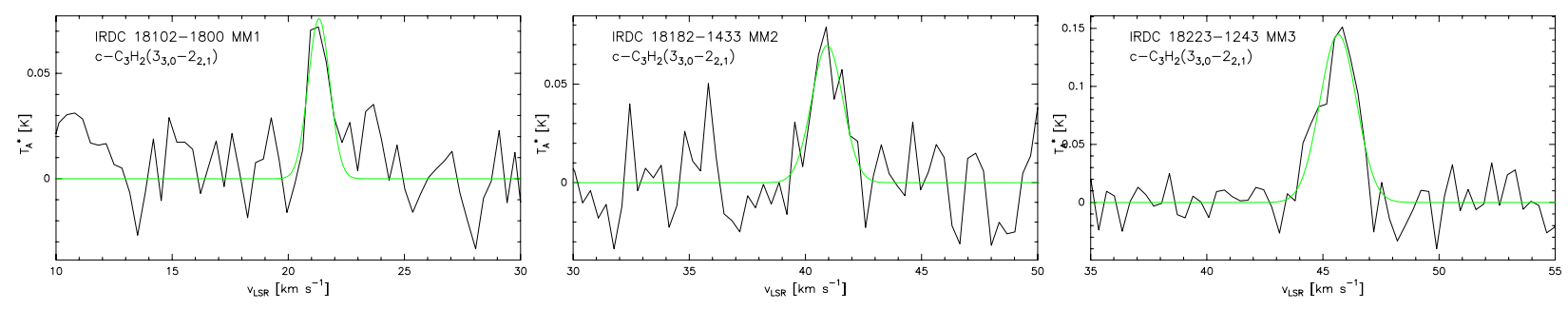

Fig. 4. ortho-c- $\mathrm{C}_{3} \mathrm{H}_{2}\left(3_{3,0}-2_{2,1}\right)$ spectra overlaid with Gaussian fits.
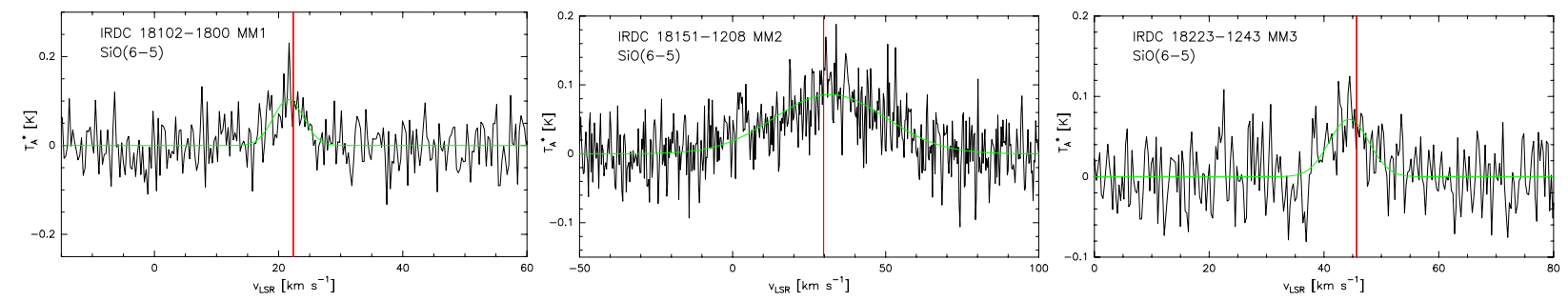

Fig. 5. $\mathrm{SiO}(6-5)$ spectra overlaid with Gaussian fits. The vertical line in each panel indicates the $v_{\mathrm{LSR}}$ of the clump as measured from $\mathrm{N}_{2} \mathrm{H}^{+}(1-0)$ by SSK08, i.e., 22.36, 29.74, and $45.73 \mathrm{~km} \mathrm{~s}^{-1}$ for I18102 MM1, I18151 MM2, and I18223 MM3, respectively. The velocity range in each panel is wider compared to that of Fig. 2 for clarity.
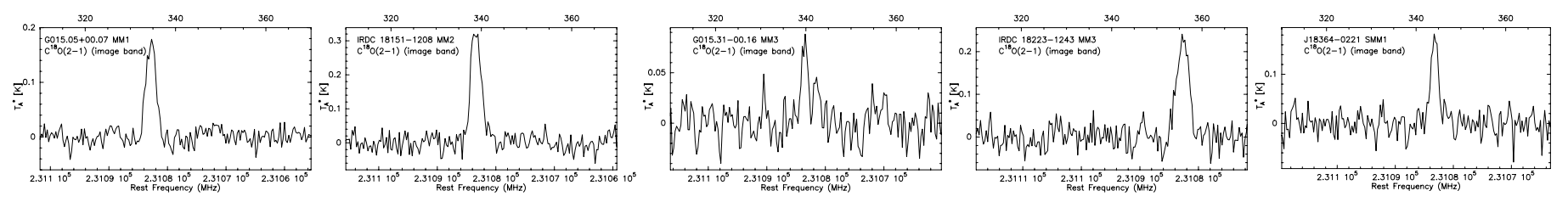

Fig. 6. $\mathrm{C}^{18} \mathrm{O}(2-1)$ lines at $219560.3568 \mathrm{MHz}$ (JPL) arising from the image sideband.

is the Planck function for a dust temperature $T_{\text {dust }}, \kappa_{v}$ is the dust opacity per unit dust mass, $R_{\mathrm{d}} \equiv\left\langle M_{\text {dust }} / M_{\text {gas }}\right\rangle$ is the average dust-to-gas mass ratio, $\mu_{\mathrm{H}_{2}}$ is the mean molecular weight per $\mathrm{H}_{2}$ molecule (2.8 for the $\mathrm{He} / \mathrm{H}$ abundance ratio of 0.1$)$, and $m_{\mathrm{H}}$ is the mass of a hydrogen atom.

The $I_{v}^{\text {dust }}$ values were determined from the (sub)mm maps shown as contours in Fig. 1. The angular resolutions of the maps are different: the beam size of the SCUBA $850 \mu \mathrm{m}$, Bolocam $1.1 \mathrm{~mm}$, and MAMBO $1.2 \mathrm{~mm}$ data are 14!"4, 31", and $11^{\prime \prime}$, respectively. The values of $S_{v}$ were taken from the literature as follows: for I18102 MM1, I18151 MM2, I18182 MM2, and I18223 MM3 we used the MAMBO 1.2-mm flux densities from Beuther et al. (2002a); for G015.05 MM1 and G015.31 MM3 we used the MAMBO-II 1.2-mm flux densities from Rathborne et al. (2006); for J18364 SMM1 we used the SCUBA 850- $\mu \mathrm{m}$ flux density from Birkmann et al. (2006). These flux densities are given in Col. (4) of Table 5.

As the dust temperature of the clumps we used the gas kinetic temperatures listed in Col. (2) of Table 5 and assumed that $T_{\text {dust }}=T_{\text {kin }}$. We extrapolated the values of $\kappa_{v}$ from the Ossenkopf \& Henning (1994) model for dust grains with thin ice mantles, coagulated for $10^{5} \mathrm{yr}$ at a gas density of $n_{\mathrm{H}}=n(\mathrm{H})+2 n\left(\mathrm{H}_{2}\right) \simeq$ $2 n\left(\mathrm{H}_{2}\right)=10^{6} \mathrm{~cm}^{-3}$ [their Table 1, Col. (6)]. This is expected to be a reasonable dust model for the sources within cold and dense IRDCs. At the wavelengths considered in the present work, these values are $\kappa_{850 \mu \mathrm{m}}=0.197 \mathrm{~m}^{2} \mathrm{~kg}^{-1}, \kappa_{1.1 \mathrm{~mm}}=0.121 \mathrm{~m}^{2} \mathrm{~kg}^{-1}$, and $\kappa_{1.2 \mathrm{~mm}}=0.106 \mathrm{~m}^{2} \mathrm{~kg}^{-1}$. The canonical value $1 / 100$ was adopted for $R_{\mathrm{d}}$. We note that the dust opacities are likely to be uncertain by a factor of $\gtrsim 2$ (Ossenkopf \& Henning 1994; Motte \& André 2001). For comparison, Williams et al. (2004), Enoch et al. (2006), and Rygl et al. (2010) used the same dust model as we did and interpolated the values of $\kappa_{v}$ at $850 \mu \mathrm{m}$,
$1.1 \mathrm{~mm}$, and $1.2 \mathrm{~mm}$ to be $0.154,0.114$, and $0.1 \mathrm{~m}^{2} \mathrm{~kg}^{-1}$, respectively. These are slightly lower than our values, and the difference is likely to be caused by different extrapolation methods (e.g., log-interpolation) and/or different dust emissivity index, $\beta$, used by the authors to determine the value of $\kappa_{v} \propto v^{\beta}$.

In the papers by Beuther et al. (2002a) and Rathborne et al. (2006), the reported clump sizes refer to their FWHM sizes (diameters) resulting from two-dimensional Gaussian fits. However, the integrated flux densities used to calculate the masses refer to larger clump areas, $A$. Accordingly, to calculate properly the volume-average $\mathrm{H}_{2}$ number density, $\left\langle n\left(\mathrm{H}_{2}\right)\right\rangle$, one way is to use the so-called effective radius of the clump, $R_{\text {eff }}=$ $\sqrt{A / \pi}$ (see, e.g., Miettinen \& Harju 2010). An alternative way to calculate $\left\langle n\left(\mathrm{H}_{2}\right)\right\rangle$ is to compute the amount of mass within the FWHM contour and use the FWHM radius. For sources with a Gaussian shape, the mass within the FWHM contour is a fraction $\ln 2 \simeq 0.693$ of the total mass (see Kauffmann \& Pillai 2010). Following Kauffmann \& Pillai (2010), we reduce the clump masses by the above factor and use half the FWHM size as the effective radius of the clump. The density $\left\langle n\left(\mathrm{H}_{2}\right)\right\rangle$ was then calculated using the formula

$\left\langle n\left(\mathrm{H}_{2}\right)\right\rangle=\frac{\langle\rho\rangle}{\mu_{\mathrm{H}_{2}} m_{\mathrm{H}}}$,

where $\langle\rho\rangle=M /\left(4 / 3 \pi R_{\text {eff }}^{3}\right)$ is the mass density, and $M$ refers to the reduced clump mass as described above. For the source J18364 SMM1 we scaled the effective radius $\approx 0.2$ pc reported by Birkmann et al. (2006) using the revised distance $\left(R_{\text {eff }}=\right.$ $0.23 \mathrm{pc}$ ), and used the total mass within the clump area.

The results of the above calculations are presented in Cols. (5)-(8) of Table 5. The uncertainties in the derived quantities were propagated from the uncertainties in $d$ and $T_{\text {kin }}$, but we 
Table 4. Spectral line parameters.

\begin{tabular}{|c|c|c|c|c|c|c|c|}
\hline Source & Transition & $\begin{array}{c}v_{\mathrm{LSR}} \\
{\left[\mathrm{km} \mathrm{s}^{-1}\right]}\end{array}$ & $\begin{array}{c}\Delta v \\
{\left[\mathrm{~km} \mathrm{~s}^{-1}\right]}\end{array}$ & $\begin{array}{l}T_{\mathrm{A}}^{*} \\
{[\mathrm{~K}]}\end{array}$ & $\int_{\left[\mathrm{K} \mathrm{km} \mathrm{s}^{-1}\right]} T_{\mathrm{A}}^{*} \mathrm{~d} v^{a}$ & $\tau_{0}^{b}$ & $\begin{array}{c}T_{\text {ex }}{ }^{2} \\
{[\mathrm{~K}]}\end{array}$ \\
\hline \multirow[t]{8}{*}{ IRDC 18102-1800 MM1 } & $\mathrm{C}^{17} \mathrm{O}(2-1)$ & $21.5 \pm 0.02$ & $2.36 \pm 0.06$ & $0.85 \pm 0.06$ & $2.58 \pm 0.05[18.4,25.0]$ & $0.08 \pm 0.01$ & $18.5 \pm 0.4$ \\
\hline & $\mathrm{H}^{13} \mathrm{CO}^{+}(3-2)$ & $21.7 \pm 0.08$ & $2.92 \pm 0.53$ & $0.37 \pm 0.05$ & $1.25 \pm 0.06[18.5,24.2]$ & $0.99 \pm 0.45$ & $4.6 \pm 0.3$ \\
\hline & $\mathrm{N}_{2} \mathrm{H}^{+}(3-2)$ & $21.9 \pm 0.02$ & $3.56 \pm 0.02$ & $2.47 \pm 0.27$ & $17.62 \pm 0.12[15.6,28.6]$ & $16.92 \pm 7.28$ & $8.2 \pm 0.1$ \\
\hline & $\mathrm{N}_{2} \mathrm{D}^{+}(3-2)$ & $22.0 \pm 0.1$ & $0.80 \pm 0.20$ & $0.07 \pm 0.01$ & $0.07 \pm 0.01[21.5,23.0]$ & 0.06 & $8.2 \pm 0.1^{d}$ \\
\hline & $\mathrm{c}-\mathrm{C}_{3} \mathrm{H}_{2}\left(3_{3,0}-2_{2,1}\right)$ & $21.3 \pm 0.1$ & $1.10 \pm 0.26$ & $0.08 \pm 0.01$ & $0.07 \pm 0.02[20.6,22.4]$ & $0.45 \pm 0.19$ & $3.4 \pm 0.1$ \\
\hline & $\mathrm{CH}_{3} \mathrm{NH}_{2}\left(7_{2,5}-7_{1,5}\right)^{e}$ & $21.1 \pm 0.2$ & $5.07 \pm 0.58$ & $0.05 \pm 0.01$ & $0.24 \pm 0.03[16.7,25.1]$ & 0.003 & 50.4 \\
\hline & ${ }^{13} \mathrm{CS}(5-4)$ & $22.3 \pm 0.2$ & $2.69 \pm 0.38$ & $0.05 \pm 0.01$ & $0.18 \pm 0.02[19.7,24.7]$ & $0.07 \pm 0.01$ & $4.7 \pm 0.2$ \\
\hline & $\mathrm{SiO}(6-5)$ & $21.5 \pm 0.4$ & $6.66 \pm 1.28$ & $0.11 \pm 0.04$ & $1.30 \pm 0.11[16.5,25.3]$ & $0.42 \pm 0.27$ & $4.0 \pm 0.7$ \\
\hline \multirow{5}{*}{ G015.05+00.07 MM1 } & $\mathrm{C}^{17} \mathrm{O}(2-1)$ & $24.6 \pm 0.03$ & $2.22 \pm 0.24$ & $0.74 \pm 0.04$ & $2.05 \pm 0.05[21.6,26.9]$ & $0.08 \pm 0.01$ & $16.4 \pm 1.7$ \\
\hline & $\mathrm{H}^{13} \mathrm{CO}^{+}(3-2)$ & $24.5 \pm 0.1$ & $1.66 \pm 0.24$ & $0.08 \pm 0.01$ & $0.20 \pm 0.02[22.8,26.8]$ & $0.11 \pm 0.01$ & $4.9 \pm 0.1$ \\
\hline & $\mathrm{DCO}^{+}(3-2)$ & $24.1 \pm 0.1$ & $0.67 \pm 0.15$ & $0.08 \pm 0.003$ & $0.07 \pm 0.01[23.3,25.2]$ & $0.09 \pm 0.01$ & $4.9 \pm 0.1$ \\
\hline & $\mathrm{N}_{2} \mathrm{H}^{+}(3-2)$ & $24.7 \pm 0.1$ & $4.58 \pm 0.29$ & $0.48 \pm 0.06$ & $2.47 \pm 0.09[19.9,29.3]$ & $0.98 \pm 0.14$ & $5.2 \pm 0.1$ \\
\hline & $\mathrm{N}_{2} \mathrm{D}^{+}(3-2)$ & $24.1 \pm 0.1$ & $0.53 \pm 0.13$ & $0.06 \pm 0.01$ & $0.03 \pm 0.01[23.4,25.4]$ & 0.12 & $5.2 \pm 0.1^{d}$ \\
\hline \multirow{8}{*}{ IRDC 18151-1208 MM2 } & $\mathrm{C}^{17} \mathrm{O}(2-1)$ & $29.5 \pm 0.02$ & $2.75 \pm 0.15$ & $1.03 \pm 0.04$ & $3.33 \pm 0.05[26.5,32.8]$ & $0.09 \pm 0.01$ & $20.8 \pm 1.9$ \\
\hline & $\mathrm{H}^{13} \mathrm{CO}^{+}(3-2)$ & $29.5 \pm 0.04$ & $2.47 \pm 0.11$ & $0.64 \pm 0.05$ & $1.63 \pm 0.06[27.4,31.8]$ & $0.44 \pm 0.10$ & $6.8 \pm 0.4$ \\
\hline & $\mathrm{DCO}^{+}(3-2)$ & $29.4 \pm 0.03$ & $1.43 \pm 0.10$ & $0.33 \pm 0.03$ & $0.75 \pm 0.03[27.3,31.3]$ & $0.15 \pm 0.03$ & $7.2 \pm 0.6$ \\
\hline & $\mathrm{N}_{2} \mathrm{D}^{+}(3-2)$ & $29.4 \pm 0.1$ & $1.03 \pm 0.18$ & $0.11 \pm 0.03$ & $0.27 \pm 0.03[28.3,30.9]$ & 0.04 & $14.0 \pm 2.0^{f}$ \\
\hline & $\mathrm{C}_{2} \mathrm{D}(3-2)^{g}$ & $29.6 \pm 0.3$ & $2.68 \pm 0.77$ & $0.07 \pm 0.02$ & $0.45 \pm 0.07^{h}$ & 0.02 & 20.8 \\
\hline & $\mathrm{CH}_{2} \mathrm{CHC}^{15} \mathrm{~N}\left(25_{2,24}-25_{0,25}\right)^{g}$ & $30.1 \pm 0.5$ & $3.15 \pm 0.97$ & $0.04 \pm 0.02$ & $0.14 \pm 0.01[27.8,33.3]$ & 0.001 & 101.2 \\
\hline & $\mathrm{U} 216452.511$ & $29.4 \pm 0.2$ & $2.06 \pm 0.49$ & $0.05 \pm 0.02$ & $0.13 \pm 0.02[28.0,31.3]$ & & \\
\hline & $\mathrm{SiO}(6-5)$ & $30.5 \pm 1.0$ & $46.80 \pm 2.36$ & $0.09 \pm 0.03$ & $1.93 \pm 0.20[-6.1,72.6]$ & $0.09 \pm 0.01$ & $5.7 \pm 0.1$ \\
\hline \multirow[t]{4}{*}{ G015.31-00.16 MM3 } & $\mathrm{C}^{17} \mathrm{O}(2-1)$ & $31.0 \pm 0.03$ & $0.80 \pm 0.09$ & $0.41 \pm 0.07$ & $0.80 \pm 0.04[28.9,32.3]$ & $0.10 \pm 0.01$ & $10.2 \pm 0.6$ \\
\hline & $\mathrm{H}^{13} \mathrm{CO}^{+}(3-2)$ & $31.4 \pm 0.2$ & $0.89 \pm 0.14$ & $0.03 \pm 0.01$ & $0.05 \pm 0.02[30.2,32.5]$ & $0.18 \pm 0.01$ & $3.5 \pm 0.1$ \\
\hline & $\mathrm{DCO}^{+}(3-2)$ & $30.8 \pm 0.2$ & $0.87 \pm 0.15$ & $0.03 \pm 0.01$ & $0.06 \pm 0.01[29.8,31.9]$ & $0.13 \pm 0.02$ & $3.4 \pm 0.1$ \\
\hline & $\mathrm{N}_{2} \mathrm{H}^{+}(3-2)$ & $30.9 \pm 0.04$ & $0.86 \pm 0.18$ & $0.26 \pm 0.02$ & $0.31 \pm 0.02[29.9,31.9]^{i}$ & $4.64 \pm 1.30$ & $3.9 \pm 0.1$ \\
\hline \multirow[t]{5}{*}{ IRDC 18182-1433 MM2 } & $\mathrm{C}^{17} \mathrm{O}(2-1)$ & $41.0 \pm 0.02$ & $1.32 \pm 0.08$ & $0.74 \pm 0.08$ & $1.82 \pm 0.03[38.0,43.0]$ & $0.13 \pm 0.01$ & $12.6 \pm 0.5$ \\
\hline & $\mathrm{H}^{13} \mathrm{CO}^{+}(3-2)$ & $40.7 \pm 0.1$ & $1.20 \pm 0.34$ & $0.11 \pm 0.02$ & $0.22 \pm 0.02[39.4,42.5]$ & $0.50 \pm 0.03$ & $3.8 \pm 0.1$ \\
\hline & $\mathrm{DCO}^{+}(3-2)$ & $41.1 \pm 0.3$ & $2.08 \pm 0.90$ & $0.09 \pm 0.01$ & $0.20 \pm 0.03[38.6,43.3]$ & $0.28 \pm 0.02$ & $3.8 \pm 0.1$ \\
\hline & $\mathrm{c}-\mathrm{C}_{3} \mathrm{H}_{2}\left(3_{3,0}-2_{2,1}\right)$ & $40.9 \pm 0.1$ & $1.58 \pm 0.31$ & $0.07 \pm 0.01$ & $0.12 \pm 0.02[39.3,42.4]$ & $1.29 \pm 0.13$ & $3.1 \pm 0.1$ \\
\hline & $\mathrm{CH}_{3} \mathrm{COCH}_{3}\left(15_{9,7}-14_{8,6}\right) ?^{j}$ & $41.3 \pm 0.1$ & $1.80 \pm 0.14$ & $0.17 \pm 0.01$ & $0.35 \pm 0.03[39.5,42.9]$ & 0.01 & 63.9 \\
\hline \multirow[t]{5}{*}{ IRDC 18223-1243 MM3 } & $\mathrm{C}^{17} \mathrm{O}(2-1)$ & $45.5 \pm 0.04$ & $3.09 \pm 0.10$ & $0.56 \pm 0.04$ & $1.97 \pm 0.05[42.1,49.0]$ & $0.06 \pm 0.01$ & $18.2 \pm 1.1$ \\
\hline & $\mathrm{H}^{13} \mathrm{CO}^{+}(3-2)$ & $45.5 \pm 0.04$ & $1.81 \pm 0.26$ & $0.39 \pm 0.03$ & $0.83 \pm 0.04[43.4,47.0]$ & $0.47 \pm 0.05$ & $5.5 \pm 0.1$ \\
\hline & $\mathrm{DCO}^{+}(3-2)$ & $45.9 \pm 0.1$ & $1.83 \pm 0.18$ & $0.11 \pm 0.01$ & $0.23 \pm 0.02[43.4,47.6]$ & $0.08 \pm 0.01$ & $5.6 \pm 0.2$ \\
\hline & $\mathrm{N}_{2} \mathrm{D}^{+}(3-2)$ & $45.3 \pm 0.3$ & $1.13 \pm 0.63$ & $0.08 \pm 0.02$ & $0.20 \pm 0.03[44.2,47.7]$ & 0.05 & $9.4 \pm 0.3^{f}$ \\
\hline & $\mathrm{c}-\mathrm{C}_{3} \mathrm{H}_{2}\left(3_{3,0}-2_{2,1}\right)$ & $45.7 \pm 0.1$ & $1.84 \pm 0.15$ & $0.15 \pm 0.03$ & $0.22 \pm 0.02[43.6,47.0]$ & $0.38 \pm 0.05$ & $4.0 \pm 0.1$ \\
\hline \multirow{2}{*}{ ISOSS J18364-0221 SMM1 } & $\mathrm{H}^{13} \mathrm{CO}^{+}(3-2)$ & $34.7 \pm 0.05$ & $0.86 \pm 0.14$ & $0.30 \pm 0.04$ & $0.44 \pm 0.04[33.5,36.1]$ & $1.07 \pm 0.38$ & $4.3 \pm 0.2$ \\
\hline & $\mathrm{DCO}^{+}(3-2)$ & $34.9 \pm 0.03$ & $1.14 \pm 0.10$ & $0.33 \pm 0.02$ & $0.50 \pm 0.02[33.5,36.3]$ & $0.69 \pm 0.20$ & $4.4 \pm 0.2$ \\
\hline
\end{tabular}

Notes. ${ }^{(a)}$ Intensities are integrated over the velocity range given in square-brackets. In the cases of I18102 MM1, I18151 MM2, and I18223 MM3, 92.5\% of the total $\mathrm{N}_{2} \mathrm{D}^{+}(3-2)$ hf component's intensity lie within the quoted velocity range. For G015.05 MM1 the fraction is $81.7 \%$. (b) For $\mathrm{C}^{17} \mathrm{O}, \mathrm{H}^{13} \mathrm{CO}^{+}, \mathrm{DCO}^{+}, \mathrm{N}_{2} \mathrm{H}^{+}$, and $\mathrm{N}_{2} \mathrm{D}^{+} \tau_{0}$ is the optical thickness in the centre of a hypothetical unsplit line. For $\mathrm{C}_{2} \mathrm{D} \tau_{0}$ is the sum of the peak optical thicknesses of all the hf components. ${ }^{(c)}$ See Sect. 4.2 for details on $T_{\text {ex }}$ determination. ${ }^{(d)} T_{\text {ex }}$ is assumed to be the same as for $\mathrm{N}_{2} \mathrm{H}^{+}(3-2)$. ${ }^{(e)}$ Another candidate for this spectral line is the OCS(19-18) transition. Alternatively, the two lines could be blended. It could also be caused by $\mathrm{C}^{18} \mathrm{O}(2-1)$ seen in the image band. ${ }^{(f)} T_{\text {ex }}\left[\mathrm{N}_{2} \mathrm{H}^{+}(1-0)\right]$ from SSK08. ${ }^{(g)}$ Another detected hf group of $\mathrm{C}_{2} \mathrm{D}(3-2)$ is blended with $\mathrm{CH}_{2} \mathrm{CHC}^{15} \mathrm{~N}\left(25_{2,24}-25_{0,25}\right)$. ${ }^{(h)}$ Integrated intensity over the two detected hf groups between [27.7, 30.7] and [-49.5, -46.5$] \mathrm{km} \mathrm{s}^{-1}$. (i) $92.6 \%$ of the hf component's intensity lie within the quoted velocity range. ${ }^{(j)}$ This line may also be caused by $\mathrm{C}^{18} \mathrm{O}(2-1)$ seen in the image band.

note that the uncertainty in (sub)mm dust opacity is the largest source of error in $M$ and $N\left(\mathrm{H}_{2}\right)$.

The derived values of $M, N\left(\mathrm{H}_{2}\right)$, and $\left\langle n\left(\mathrm{H}_{2}\right)\right\rangle$ are mostly within a factor of $\lesssim 2$ of those derived by Beuther et al. $(2002 \mathrm{a}$; where the revised equations by Beuther et al. 2005b, are employed), Rathborne et al. (2006), Birkmann et al. (2006), and Hennemann et al. (2009). We note that besides the different source distances, the dust parameters used by the authors in the above reference studies were different than here. Beuther et al. (2002a, 2005b) used $T_{\text {dust }}$ values $(35-50 \mathrm{~K})$ resulting from the cold part of the source SEDs; the SEDs were constructed from the IRAS and $\mathrm{mm}$ data of the main core in the source region, which explains the rather high dust temperatures. In addition, Beuther et al. (2002a, 2005b) used the grain radius, mass density, and emissivity index values of $a=0.1 \mu \mathrm{m}, \rho_{\text {dust }}=3 \mathrm{~g} \mathrm{~cm}^{-3}$, and $\beta=2$, respectively, in the calculation of mass and column density. Following Hildebrand (1983), these values correspond to $\kappa_{1.2 \mathrm{~mm}} \approx 0.5 \mathrm{~m}^{2} \mathrm{~kg}^{-1}$. Rathborne et al. (2006) used the values $T_{\text {dust }}=15 \mathrm{~K}$ and $\kappa_{1.2 \mathrm{~mm}}=0.1 \mathrm{~m}^{2} \mathrm{~kg}^{-1}$. For the source $\mathrm{J} 18364$
SMM1, Birkmann et al. (2006) used $T_{\text {dust }}=16.5_{-3.0}^{+6.0} \mathrm{~K}$ instead of $T_{\text {kin }}$, and they had $\kappa_{850 \mu \mathrm{m}}=0.180 \mathrm{~m}^{2} \mathrm{~kg}^{-1}$, which is slightly smaller than ours even though the adopted dust model was the same.

\subsection{Molecular column densities and fractional abundances}

The beam-averaged column densities of $\mathrm{C}^{17} \mathrm{O}, \mathrm{H}^{13} \mathrm{CO}^{+}, \mathrm{DCO}^{+}$, $\mathrm{N}_{2} \mathrm{H}^{+}, \mathrm{SiO}, \mathrm{OCS},{ }^{13} \mathrm{CS}$, and $o-\mathrm{c}-\mathrm{C}_{3} \mathrm{H}_{2}$ were derived using a one-dimensional spherically symmetric non-LTE radiative transfer code called RADEX (see Sect. 3.2). RADEX uses the method of mean escape probability for an isothermal and homogeneous medium. The molecular data files (collisional rates) used in the RADEX excitation analysis were taken from the LAMDA database (Schöier et al. 2005). The $\mathrm{C}^{17} \mathrm{O}, \mathrm{H}^{13} \mathrm{CO}^{+}, \mathrm{DCO}^{+}$, and $\mathrm{N}_{2} \mathrm{H}^{+}$transitions are treated as a hypothetical unsplit transition. The input parameters in the off-line mode of RADEX are the gas kinetic temperature, $\mathrm{H}_{2}$ number density, and the width (FWHM) and intensity of the spectral line. We used the values of $T_{\text {kin }}$ and 
Table 5. Physical properties of the sources.

\begin{tabular}{lccccccc}
\hline \hline Source & $\begin{array}{c}T_{\text {kin }}{ }^{a} \\
{[\mathrm{~K}]}\end{array}$ & $\begin{array}{c}T_{\text {dust }}{ }^{b} \\
{[\mathrm{~K}]}\end{array}$ & $\begin{array}{c}S_{v}{ }^{c} \\
{[\mathrm{Jy}]}\end{array}$ & $\begin{array}{c}M \\
{\left[\mathrm{M}_{\odot}\right]}\end{array}$ & $\begin{array}{c}N\left(\mathrm{H}_{2}\right) \\
{\left[10^{22} \mathrm{~cm}^{-2}\right]}\end{array}$ & $\begin{array}{c}R_{\text {eff }}[\mathrm{pc}] \\
\left.\left[10^{4} \mathrm{H}_{2}\right)\right\rangle \\
{\left[10^{4} \mathrm{~cm}^{-3}\right]}\end{array}$ \\
\hline IRDC 18102-1800 MM1 & $21.3 \pm 1.6$ & $\ldots$ & 3.3 & $357 \pm 163$ & $6.5 \pm 0.7$ & 0.34 & $2.9 \pm 1.3$ \\
G015.05+00.07 MM1 & $17.2 \pm 2.1$ & $11.0-36.0$ & 0.47 & $63 \pm 31$ & $1.0 \pm 0.2$ & 0.15 & $5.9 \pm 2.0$ \\
IRDC 18151-1208 MM2 & $21.1 \pm 2.0$ & $\ldots$ & 2.6 & $285 \pm 111$ & $12.9 \pm 1.6$ & 0.17 & $18.5 \pm 7.2$ \\
G015.31-00.16 MM3 & $13.7 \pm 2.8$ & $\ldots$ & 0.74 & $183 \pm 83$ & $0.8 \pm 0.3$ & 0.38 & $1.1 \pm 0.5$ \\
IRDC 18182-1433 MM2 & $15.3 \pm 1.5$ & $\ldots$ & 0.3 & $86 \pm 23$ & $5.2 \pm 0.8$ & 0.26 & $1.6 \pm 0.4$ \\
IRDC 18223-1243 MM3 & $18.7 \pm 1.3$ & $15.0 / 18.0$ & 0.8 & $173 \pm 43$ & $1.4 \pm 0.1$ & 0.18 & $9.5 \pm 2.3$ \\
ISOSS J18364-0221 SMM1 & 11.4 & $16.5_{-3.0}^{+6.0}$ & 2.11 & $169 \pm 54$ & 7.6 & 0.23 & $6.4 \pm 2.0$ \\
\hline
\end{tabular}

Notes. ${ }^{(a)}$ Calculated from $T_{\text {rot }}\left(\mathrm{NH}_{3}\right)$ from Krause (2003) and SSK08. Krause (2003) reported a slightly higher $T_{\text {kin }}$ value of $11.8 \mathrm{~K}$ for J18364 SMM1. ${ }^{(b)}$ For G015.05 MM1, $T_{\text {dust }}$ is from Rathborne et al. (2010). For I18223 MM3, the two $T_{\text {dust }}$ values, 15 and $18 \mathrm{~K}$, are from Beuther \& Steinacker (2007) and Beuther et al. (2010), respectively. The $T_{\text {dust }}$ value for J18364 SMM1 is from Birkmann et al. (2006). ${ }^{(c)}$ For those sources whose name start with "IRDC", $S_{v}$ refers to MAMBO 1.2-mm flux density from Beuther et al. (2002a). For G015.05 MM1 and G015.31 MM3, the quoted value is the MAMBO-II 1.2-mm flux density (Rathborne et al. 2006). In the case of J18364 SMM1, we give the SCUBA 850- $\mu$ m flux density from Birkmann et al. (2006).

$\left\langle n\left(\mathrm{H}_{2}\right)\right\rangle$ listed in Table 5. However, we multiplied the densities by $1.2\left(\mathrm{He} / \mathrm{H}_{2}=0.2\right)$ to take the collisions with He into account (see Sect. 4.1 of the RADEX manual ${ }^{12}$ ). As the input line intensity we used the main-beam brightness temperature $T_{\mathrm{MB}}=T_{\mathrm{A}}^{*} / \eta_{\mathrm{MB}}$. When the source is resolved, $T_{\mathrm{MB}}$ is equal to the Rayleigh-Jeans equivalent radiation temperature, $T_{\mathrm{R}}$. The simulations aim to reproduce the observed line intensity and yield the values of the line peak optical thickness $\left(\tau_{0}\right)$, excitation temperature $\left(T_{\text {ex }}\right)$, and the total column density of the molecule $\left(N_{\text {tot }}\right)$. We varied $T_{\text {kin }}$ and $\left\langle n\left(\mathrm{H}_{2}\right)\right\rangle$ according to their errors to estimate the uncertainties associated with $\tau_{0}, T_{\mathrm{ex}}$, and $N_{\text {tot }}$. The lines appear to be optically thin in most cases. The optical thickness is $\tau_{0} \gtrsim 1$ for all $\mathrm{N}_{2} \mathrm{H}^{+}$ lines, for $\mathrm{H}^{13} \mathrm{CO}^{+}$towards I18102 MM1 and J18364 SMM1, and for $\mathrm{c}_{-} \mathrm{C}_{3} \mathrm{H}_{2}$ of I18182 MM2. The $T_{\mathrm{ex}}$ values for $\mathrm{C}^{17} \mathrm{O}$ are close to $T_{\text {kin }}$, indicating that the lines are nearly thermalised. Also, $T_{\mathrm{ex}}\left(\mathrm{H}^{13} \mathrm{CO}^{+}\right)$is found to be equal to $T_{\mathrm{ex}}\left(\mathrm{DCO}^{+}\right)$within the errors. We note that the column density determination is in some cases hampered by self-absorbed line profiles.

For the rest of the observed molecules there are no molecular data files available in the LAMDA database. The line optical thicknesses and total beam-averaged column densities of these molecules were determined through LTE modelling with CLASS/Weeds. The input parameters for a Weeds model are $N_{\text {tot }}, T_{\text {ex }}$, source size $\left(\theta_{\mathrm{s}}\right)$, linewidth (FWHM), and offset from the reference-channel velocity. The linewidth is directly determined from the observed line profile, so there are basically three free parameters left $\left(N_{\mathrm{tot}}, T_{\mathrm{ex}}, \theta_{\mathrm{s}}\right)$. Some of the model parameters may be degenerate, and cannot be determined independently (Schilke et al. 2006; Maret et al. 2011). The source size is degenerate with excitation temperature for completely optically thick lines $(\tau \gg 1)$, and with column density if the lines are completely optically thin $(\tau \ll 1)$. We assumed that the source fills the telescope beam, i.e., that the source solid angle, $\Omega_{\mathrm{s}} \propto \theta_{\mathrm{s}}^{2}$, equals the beam solid angle, $\Omega_{\mathrm{A}} \propto \theta_{\mathrm{HPBW}}^{2}$, or $\theta_{\mathrm{s}}=\theta_{\mathrm{HPBW}}$. When the beam filling factor is unity, the line brightness temperature is $T_{\mathrm{B}} \simeq T_{\mathrm{MB}}$ (see Eqs. (1) and (2) in Maret et al. 2011). For $\mathrm{N}_{2} \mathrm{D}^{+}$, we used as $T_{\text {ex }}$ the values obtained for $\mathrm{N}_{2} \mathrm{H}^{+}$from RADEX simulations or from SSK08 (Sect. 3.2). For the rest of the transitions we adopted the value $T_{\mathrm{ex}}=E_{\mathrm{u}} / k_{\mathrm{B}}$ for linear rotors, and $T_{\mathrm{ex}}=2 / 3 \times E_{\mathrm{u}} / k_{\mathrm{B}}$ for asymmetric top rotors. The adopted rotational excitation temperatures give a lower limit to the total beam-averaged column densities (Hatchell et al. 1998). The

\footnotetext{
12 http://www.sron.rug.nl/ vdtak/radex/radex_manual. $\operatorname{pdf}$
}

input $N_{\text {tot }}$ was then varied until a reasonable fit to the line was obtained (see Fig. 7). We note that we detected only one transition per species for the complex organics. We therefore cannot apply a rotation diagram to derive rotational temperatures and molecular column densities (e.g., Goldsmith \& Langer 1999).

We also determined the $\mathrm{HCO}^{+}$column density from the column density of $\mathrm{H}^{13} \mathrm{CO}^{+}$. For this calculation, it was assumed that the carbon-isotope ratio $\left[{ }^{12} \mathrm{C}\right] /\left[{ }^{13} \mathrm{C}\right]$ depends on $R_{\mathrm{GC}}$ according to the relationship given by Wilson \& Rood (1994):

$$
\frac{\left[{ }^{12} \mathrm{C}\right]}{\left[{ }^{13} \mathrm{C}\right]}=7.5 \times R_{\mathrm{GC}}[\mathrm{kpc}]+7.6 .
$$

For the $R_{\mathrm{GC}}$ values considered here, $5.1-6.3 \mathrm{kpc}$, the above ratio lies in the range $\sim 46-55^{13}$.

For I18151 MM2, I18182 MM2, and I18223 MM3 we do not have $\mathrm{N}_{2} \mathrm{H}^{+}$data. For these sources we computed the $\mathrm{N}_{2} \mathrm{H}^{+}$column density from the $J=1-0$ line parameters $\left(T_{\mathrm{ex}}\right.$ and $\left.\tau\right)$ from SSK08 (see, e.g., Eq. (10) in Miettinen et al. 2010). We obtain about 1.1-1.2 times higher $\mathrm{N}_{2} \mathrm{H}^{+}$column densities compared to SSK08, who used the optically thin approximation (see Sect. 5.1 for a more detailed discussion).

We calculated the fractional abundances of the molecules by dividing the molecular column density by the $\mathrm{H}_{2}$ column density: $x(\mathrm{~mol})=N(\mathrm{~mol}) / N\left(\mathrm{H}_{2}\right)$. For this purpose, the values of $N\left(\mathrm{H}_{2}\right)$ were derived from the (sub)mm dust continuum maps smoothed to the corresponding resolution of the line observations. With a resolution of 31", the Bolocam 1.1-mm data could not be smoothed to correspond the resolution of the line observations. In these cases we used the original Bolocam data; the 31" resolution is in most cases comparable to that of the line observations (22.'3-28.'9), and therefore we do not expect this to be a significant source of error. The derived column densities and abundances are listed in Tables 6 and 7. The abundance errors were derived by propagating the errors in $N(\mathrm{~mol})$ and $N\left(\mathrm{H}_{2}\right)$.

\footnotetext{
${ }^{13}$ Spectral lines of ${ }^{12} \mathrm{C}$-isotopologue of $\mathrm{HCO}^{+}$are likely to be optically thick. Therefore, the $\mathrm{HCO}^{+}$deuteration can be better investigated through the $\mathrm{DCO}^{+} / \mathrm{H}^{13} \mathrm{CO}^{+}$column density ratio. However, a caveat should be noted here. The $\mathrm{HCO}^{+}$molecules are produced directly from CO (see reactions 3 and 7 in Table 8). On the other hand, at low temperature $\mathrm{CO}$ is susceptible to the exothermic isotopic charge exchange reaction ${ }^{13} \mathrm{C}^{+}+{ }^{12} \mathrm{CO} \rightarrow{ }^{12} \mathrm{C}^{+}+{ }^{13} \mathrm{CO}+\Delta E$, where $\Delta E / k_{\mathrm{B}}=$ $35 \mathrm{~K}$ (Watson et al. 1976). This is expected to cause considerable ${ }^{13} \mathrm{C}$ fractionation in cold and dense gas, which complicates the deuteration analysis.
} 
Table 6. Molecular column densities and fractional abundances with respect to $\mathrm{H}_{2}$.

\begin{tabular}{lcccccc}
\hline \hline Source & $\begin{array}{c}N\left(\mathrm{C}^{17} \mathrm{O}\right) \\
{\left[10^{15} \mathrm{~cm}^{-2}\right]}\end{array}$ & $\begin{array}{c}N\left(\mathrm{~N}_{2} \mathrm{H}^{+}\right) \\
{\left[10^{13} \mathrm{~cm}^{-2}\right]}\end{array}$ & $\begin{array}{c}N\left(\mathrm{~N}_{2} \mathrm{D}^{+}\right) \\
{\left[10^{11} \mathrm{~cm}^{-2}\right]}\end{array}$ & $\begin{array}{c}N\left(\mathrm{H}^{13} \mathrm{CO}^{+}\right) \\
{\left[10^{13} \mathrm{~cm}^{-2}\right]}\end{array}$ & $\begin{array}{c}N\left(\mathrm{HCO}^{+}\right) \\
{\left[10^{14} \mathrm{~cm}^{-2}\right]}\end{array}$ & $\begin{array}{c}N\left(\mathrm{DCO}^{+}\right) \\
{\left[10^{11} \mathrm{~cm}^{-2}\right]}\end{array}$ \\
\hline IRDC 18102-1800 MM1 & $1.4 \pm 0.1$ & $30.1 \pm 13.3$ & 4.9 & $1.9 \pm 1.0$ & $9.7 \pm 5.1$ & $1.9 \pm 0.9$ \\
G015.05+00.07 MM1 & $1.2 \pm 0.1$ & $3.3 \pm 0.6$ & 9.2 & $0.1 \pm 0.03$ & $0.5 \pm 0.2$ & $3.0 \pm 0.6$ \\
IRDC 18151-1208 MM2 & $2.1 \pm 0.1$ & $5.0 \pm 1.1^{a}$ & 5.2 & $0.5 \pm 0.1$ & $2.6 \pm 0.5$ & $8.2 \pm 1.9$ \\
G015.31-00.16 MM3 & $0.3 \pm 0.1$ & $3.8 \pm 0.6$ & $\ldots$ & $0.3 \pm 0.04$ & $1.5 \pm 0.2$ & $11.0 \pm 2.1$ \\
IRDC 18182-1433 MM2 & $0.7 \pm 0.1$ & $2.1 \pm 0.4^{a}$ & $\ldots$ & $0.7 \pm 0.1$ & $3.2 \pm 0.5$ & $45.4 \pm 5.4$ \\
IRDC 18223-1243 MM3 & $1.2 \pm 0.1$ & $5.1 \pm 0.5^{a}$ & 6.7 & $0.4 \pm 0.1$ & $1.9 \pm 0.5$ & $6.6 \pm 0.9$ \\
ISOSS J18364-0221 SMM1 & $1.1 \pm 0.1$ & $\ldots$ & $\ldots$ & $0.6 \pm 0.2$ & $3.3 \pm 1.1$ & $39.2 \pm 13.8$ \\
\hline & $x\left(\mathrm{C}^{17} \mathrm{O}\right)$ & $x\left(\mathrm{~N}_{2} \mathrm{H}^{+}\right)$ & $x\left(\mathrm{~N}_{2} \mathrm{D}^{+}\right)$ & $x\left(\mathrm{H}^{13} \mathrm{CO}^{+}\right)$ & $x\left(\mathrm{HCO}^{+}\right)$ & $x\left(\mathrm{DCO}^{+}\right)$ \\
& {$\left[10^{-7}\right]$} & {$\left[10^{-9}\right]$} & {$\left[10^{-11}\right]$} & {$\left[10^{-10}\right]$} & {$\left[10^{-8}\right]$} & {$\left[10^{-11}\right]$} \\
\hline IRDC 18102-1800 MM1 & $1.5 \pm 0.2$ & $27.7 \pm 12.6$ & $5.0 \pm 0.5$ & $18.2 \pm 9.8$ & $9.3 \pm 5.0$ & $2.0 \pm 1.0$ \\
G015.05+00.07 MM1 & $1.2 \pm 0.2$ & $3.4 \pm 0.9$ & $9.5 \pm 1.7$ & $1.0 \pm 0.4$ & $0.5 \pm 0.2$ & $3.1 \pm 0.8$ \\
IRDC 18151-1208 MM2 & $0.6 \pm 0.1$ & $0.9 \pm 0.2$ & $1.3 \pm 0.2$ & $1.2 \pm 0.3$ & $0.6 \pm 0.1$ & $2.3 \pm 0.6$ \\
G015.31-00.16 MM3 & $0.4 \pm 0.2$ & $4.5 \pm 1.6$ & $\ldots$ & $3.5 \pm 1.2$ & $1.8 \pm 0.6$ & $13.0 \pm 4.8$ \\
IRDC 18182-1433 MM2 & $1.1 \pm 0.2$ & $2.5 \pm 0.6$ & $\ldots$ & $9.9 \pm 2.1$ & $4.5 \pm 1.0$ & $72.8 \pm 14.6$ \\
IRDC 18223-1243 MM3 & $0.9 \pm 0.1$ & $3.7 \pm 0.5^{b}$ & $4.9 \pm 0.5$ & $2.9 \pm 0.8$ & $1.4 \pm 0.4$ & $4.8 \pm 0.8$ \\
ISOSS J18364-0221 SMM1 & $1.6 \pm 0.1$ & $\ldots$ & $\ldots$ & $7.3 \pm 2.4$ & $4.0 \pm 1.3$ & $58.7 \pm 20.7$ \\
\hline
\end{tabular}

Notes. ${ }^{(a)}$ Calculated from the SSK08 parameters (18" resolution). ${ }^{(b)}$ The corresponding $\mathrm{H}_{2}$ column density was estimated from the Bolocam map of $31^{\prime \prime}$ resolution.

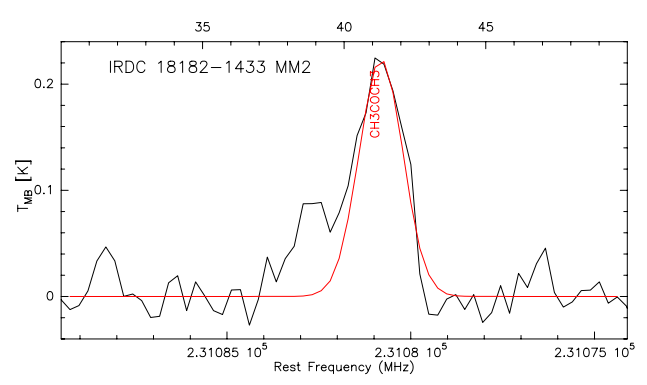

Fig. 7. Example of the Weeds LTE modelling outlined in Sect. 4.2. The synthetic model spectrum is overlaid as a red line. The intensity scale is $T_{\mathrm{MB}}$ for modelling purposes.

\section{3. $\mathrm{CO}$ depletion and deuterium fractionation}

To estimate the amount of $\mathrm{CO}$ depletion in the clumps, we calculated the $\mathrm{CO}$ depletion factor, $f_{\mathrm{D}}$, following the analysis presented in the paper by Fontani et al. (2006). If $x(\mathrm{CO})_{\text {can }}$ is the "canonical" (undepleted) abundance, and $x(\mathrm{CO})_{\mathrm{obs}}$ is the observed $\mathrm{CO}$ abundance, $f_{\mathrm{D}}$ is given by

$f_{\mathrm{D}}=\frac{x(\mathrm{CO})_{\mathrm{can}}}{x(\mathrm{CO})_{\mathrm{obs}}}$

The "canonical" $\mathrm{CO}$ abundance at the galactocentric distance $R_{\mathrm{GC}}$ was calculated using the relationship (Eq. (7) in Fontani et al. 2006)

$x(\mathrm{CO})_{\mathrm{can}}=9.5 \times 10^{-5} \mathrm{e}^{1.105-0.13 R_{\mathrm{GC}}[\mathrm{kpc}]}$.

This relationship results from using the value $R_{0}=8.5 \mathrm{kpc}$, whereas our $R_{\mathrm{GC}}$ values are computed using $R_{0}=8.4 \mathrm{kpc}$. This small discrepancy is negligible, however. At $R_{\mathrm{GC}}=8.5 \mathrm{kpc}$, the above relationship gives the standard value $9.5 \times 10^{-5}$ for the abundance of the main CO isotopologue in the solar neighbourhood (Frerking et al. 1982). To calculate the "canonical" $\mathrm{C}^{17} \mathrm{O}$ abundance we take into account that the oxygen-isotopic ratio $\left[{ }^{16} \mathrm{O}\right] /\left[{ }^{18} \mathrm{O}\right]$ depends on $R_{\mathrm{GC}}$ according to the relationship (Wilson \& Rood 1994)

$\frac{\left[{ }^{16} \mathrm{O}\right]}{\left[{ }^{18} \mathrm{O}\right]}=58.8 \times R_{\mathrm{GC}}[\mathrm{kpc}]+37.1$.
Table 7. Column densities and fractional abundances of the other candidate species observed.

\begin{tabular}{|c|c|c|c|}
\hline Source & Molecule & $N_{\text {tot }}\left[\mathrm{cm}^{-2}\right]$ & $x \equiv N_{\text {tot }} / N\left(\mathrm{H}_{2}\right)$ \\
\hline \multirow[t]{5}{*}{ IRDC 18102-1800 MM1 } & $o$-c- $\mathrm{C}_{3} \mathrm{H}_{2}$ & $1.7 \pm 0.8 \times 10^{13}$ & $1.8 \pm 0.8 \times 10^{-9}$ \\
\hline & $\mathrm{CH}_{3} \mathrm{NH}_{2}{ }^{a}$ & $5.0 \times 10^{14 b}$ & $5.1 \pm 0.6 \times 10^{-8}$ \\
\hline & $\mathrm{OCS}^{a}$ & $2.3 \pm 0.8 \times 10^{15}$ & $2.4 \pm 0.9 \times 10^{-7}$ \\
\hline & ${ }^{13} \mathrm{CS}$ & $6.0 \pm 2.9 \times 10^{13}$ & $6.2 \pm 3.1 \times 10^{-9}$ \\
\hline & $\mathrm{SiO}$ & $1.2 \pm 0.8 \times 10^{15}$ & $1.1 \pm 0.8 \times 10^{-7}$ \\
\hline \multirow[t]{4}{*}{ IRDC 18151-1208 MM2 } & $o-c-\mathrm{C}_{3} \mathrm{H}_{2}$ & $7.0 \pm 1.7 \times 10^{12}$ & $1.9 \pm 0.5 \times 10^{-10}$ \\
\hline & $\mathrm{C}_{2} \mathrm{D}^{c}$ & $1.5 \times 10^{13 d}$ & $4.2 \pm 0.5 \times 10^{-10}$ \\
\hline & $\mathrm{CH}_{2} \mathrm{CHC}^{15} \mathrm{~N}$ & $2.5 \times 10^{16 b}$ & $6.9 \pm 0.9 \times 10^{-7}$ \\
\hline & $\mathrm{SiO}$ & $1.1 \pm 0.3 \times 10^{15}$ & $2.5 \pm 0.8 \times 10^{-8}$ \\
\hline \multirow[t]{2}{*}{ IRDC 18182-1433 MM2 } & $o-\mathrm{c}-\mathrm{C}_{3} \mathrm{H}_{2}$ & $7.7 \pm 0.8 \times 10^{13}$ & $1.2 \pm 0.2 \times 10^{-8}$ \\
\hline & $\mathrm{CH}_{3} \mathrm{COCH}_{3}$ & $2.0 \times 10^{15 b}$ & $4.1 \pm 0.6 \times 10^{-7}$ \\
\hline \multirow[t]{2}{*}{ IRDC 18223-1243 MM3 } & $o-\mathrm{c}-\mathrm{C}_{3} \mathrm{H}_{2}$ & $2.2 \pm 0.3 \times 10^{13}$ & $1.6 \pm 0.3 \times 10^{-9}$ \\
\hline & $\mathrm{SiO}$ & $4.0 \pm 0.6 \times 10^{14}$ & $2.9 \pm 0.5 \times 10^{-8}$ \\
\hline
\end{tabular}

Notes. ${ }^{(a)}$ The corresponding line is blended with OCS. ${ }^{(b)}$ Calculated by assuming that $T_{\mathrm{ex}}=2 / 3 \times E_{\mathrm{u}} / k_{\mathrm{B}} .{ }^{(c)}$ The other $\mathrm{C}_{2} \mathrm{D}$ hf group detected is possibly blended with $\mathrm{CH}_{2} \mathrm{CHC}^{15} \mathrm{~N}$. ${ }^{(d)}$ Calculated by assuming that $T_{\mathrm{ex}}=E_{\mathrm{u}} / k_{\mathrm{B}}$.

For the $R_{\mathrm{GC}}$ values of our clumps the above ratio ranges from about 337 to 407.5 . When this relationship is combined with the $\left[{ }^{18} \mathrm{O}\right] /\left[{ }^{17} \mathrm{O}\right]$ ratio, for which we use the standard value 3.52 (Frerking et al. 1982), the value of $x\left(\mathrm{C}^{17} \mathrm{O}\right)_{\text {can }}$ can be calculated as

$$
\begin{aligned}
x\left(\mathrm{C}^{17} \mathrm{O}\right)_{\mathrm{can}} & =\frac{x(\mathrm{CO})_{\mathrm{can}}}{\left[{ }^{18} \mathrm{O}\right] /\left[{ }^{17} \mathrm{O}\right] \times\left[{ }^{16} \mathrm{O}\right] /\left[{ }^{18} \mathrm{O}\right]} \\
& =\frac{x(\mathrm{CO})_{\mathrm{can}}}{3.52 \times\left(58.8 \times R_{\mathrm{GC}}[\mathrm{kpc}]+37.1\right)} .
\end{aligned}
$$

The depletion factor $f_{\mathrm{D}}$ is then calculated from $f_{\mathrm{D}}=$ $x\left(\mathrm{C}^{17} \mathrm{O}\right)_{\mathrm{can}} / x\left(\mathrm{C}^{17} \mathrm{O}\right)_{\mathrm{obs}}$, and the results, $f_{\mathrm{D}}=0.6 \pm 0.1-2.7 \pm 1.3$, are listed in Col. (2) of Table 9. The \pm -error quoted was calculated by propagating the uncertainty in $x\left(\mathrm{C}^{17} \mathrm{O}\right)_{\text {obs. }}$. The low values of $f_{\mathrm{D}}$ indicate that $\mathrm{CO}$ is not significantly depleted, if at all, in our clumps.

The degree of deuterium fractionation in $\mathrm{HCO}^{+}$and $\mathrm{N}_{2} \mathrm{H}^{+}$was calculated by dividing the column density of the deuterated isotopologue by its normal hydrogen-bearing form 
Table 8. Ion-molecule reactions included in the analysis of ionisation degree.

\begin{tabular}{|c|c|c|}
\hline No. & $\overline{\text { Reaction }}$ & $\begin{array}{c}\text { Note on } \\
\text { rate coefficient }{ }^{a}\end{array}$ \\
\hline 1 & $\begin{array}{l}\mathrm{H}_{2}+\operatorname{crp} \stackrel{{ }_{\mathrm{H}_{2}}}{\longrightarrow} \mathrm{H}_{2}^{+}+\mathrm{e}^{-} \\
\mathrm{H}_{2}^{+}+\mathrm{H}_{2} \longrightarrow \mathrm{H}_{3}^{+}+\mathrm{H}\end{array}$ & $\zeta_{\mathrm{H}_{2}}\left[\mathrm{~s}^{-1}\right]$ \\
\hline 2 & $\mathrm{H}_{3}^{+}+\mathrm{HD} \underset{k_{-}}{\stackrel{k_{+}}{\rightleftharpoons}} \mathrm{H}_{2} \mathrm{D}^{+}+\mathrm{H}_{2}$ & $k_{+}$from Hugo et al. (2009) \\
\hline 3 & $\mathrm{H}_{3}^{+}+\mathrm{CO} \stackrel{k_{3}}{\longrightarrow} \mathrm{HCO}^{+}+\mathrm{H}_{2}$ & $k_{3}=1.7 \times 10^{-9} \mathrm{~cm}^{3} \mathrm{~s}^{-1}$ \\
\hline 4 & $\begin{aligned} \mathrm{H}_{3}^{+}+\mathrm{e}^{-} \stackrel{\beta_{4}}{\longrightarrow} \mathrm{H}_{2}+\mathrm{H} \\
\mathrm{H}+\mathrm{H}+\mathrm{H}\end{aligned}$ & $\beta_{4}$ from Pagani et al. (2009a) \\
\hline 5 & $\begin{array}{r}\mathrm{H}_{3}^{+}+\mathrm{O} \stackrel{k_{5}}{\longrightarrow} \mathrm{H}_{2} \mathrm{O}^{+}+\mathrm{H} \\
\mathrm{OH}^{+}+\mathrm{H}_{2}\end{array}$ & $k_{5}=1.2 \times 10^{-9} \mathrm{~cm}^{3} \mathrm{~s}^{-1}$ \\
\hline $6^{b}$ & $\begin{aligned} \mathrm{H}_{3}^{+}+\mathrm{g}^{-} \stackrel{k_{6}}{\longrightarrow} & g^{0}+\mathrm{H}_{2}+\mathrm{H} \\
& \mathrm{g}^{0}+\mathrm{H}+\mathrm{H}+\mathrm{H}\end{aligned}$ & $k_{6}$ from Pagani et al. (2009a) \\
\hline 7 & $\begin{array}{r}\mathrm{H}_{2} \mathrm{D}^{+}+\mathrm{CO} \stackrel{k_{7}}{\longrightarrow} \mathrm{HCO}^{+}+\mathrm{HD}\left(\frac{2}{3}\right) \\
\mathrm{DCO}^{+}+\mathrm{H}_{2}\left(\frac{1}{3}\right)\end{array}$ & $k_{7}=1 / 3 \times k_{3}$ \\
\hline 8 & $\begin{array}{c}\mathrm{H}_{2} \mathrm{D}^{+}+\mathrm{e}^{-} \stackrel{\beta_{8}}{\longrightarrow} \mathrm{H}+\mathrm{H}+\mathrm{D} \\
\mathrm{HD}+\mathrm{H} \\
\mathrm{H}_{2}+\mathrm{D}\end{array}$ & $\beta_{8}$ from Pagani et al. (2009a) \\
\hline 9 & $\begin{array}{r}\mathrm{H}_{2} \mathrm{D}^{+}+\mathrm{O} \stackrel{k_{9}}{\longrightarrow} \mathrm{OH}^{+}+\mathrm{HD} \\
\mathrm{OD}^{+}+\mathrm{H}_{2}\end{array}$ & $k_{9}=k_{5}$ \\
\hline 10 & $\begin{aligned} \mathrm{H}_{2} \mathrm{D}^{+}+\mathrm{g}^{-} \stackrel{k_{10}}{\longrightarrow} & \mathrm{g}^{0}+\mathrm{H}_{2}+\mathrm{D} \\
& \mathrm{g}^{0}+\mathrm{HD}+\mathrm{H} \\
& \mathrm{g}^{0}+\mathrm{D}+\mathrm{H}+\mathrm{H}\end{aligned}$ & $k_{10}$ from Pagani et al. (2009a) \\
\hline 11 & $\mathrm{HCO}^{+}+\mathrm{e}^{-} \stackrel{\beta_{11}}{\longrightarrow} \mathrm{CO}+\mathrm{H}$ & $\beta_{11}=2.4 \times 10^{-7}\left(\frac{T_{\mathrm{kin}}}{300 \mathrm{~K}}\right)^{-0.69}$ \\
\hline 12 & $\mathrm{HCO}^{+}+\mathrm{g}^{-} \stackrel{k_{12}}{\longrightarrow} \mathrm{g}^{0}+\mathrm{CO}+\mathrm{H}$ & $k_{12}$ from Pagani et al. (2009a) \\
\hline 13 & $\mathrm{DCO}^{+}+\mathrm{e}^{-} \stackrel{\beta_{13}}{\longrightarrow} \mathrm{CO}+\mathrm{D}$ & $\beta_{13}=\beta_{11}$ \\
\hline 14 & $\mathrm{DCO}^{+}+\mathrm{g}^{-} \stackrel{k_{14}}{\longrightarrow} \mathrm{g}^{0}+\mathrm{CO}+\mathrm{D}$ & $k_{14}=k_{12}$ \\
\hline 15 & $\mathrm{~N}_{2} \mathrm{H}^{+}+\mathrm{CO} \stackrel{k_{15}}{\longrightarrow} \mathrm{HCO}^{+}+\mathrm{N}_{2}$ & $k_{15}=8.8 \times 10^{-10} \mathrm{~cm}^{3} \mathrm{~s}^{-1}$ \\
\hline
\end{tabular}

Notes. ${ }^{(a)}$ The rate coefficients are taken from the UMIST database unless otherwise stated. The temperature-dependent rates were calculated by using the $T_{\text {kin }}$ values listed in Col. (2) of Table 5. ${ }^{(b)}$ The label $g$ refers to the dust grains.

as $R_{\mathrm{D}}\left(\mathrm{HCO}^{+}\right) \equiv N\left(\mathrm{DCO}^{+}\right) / N\left(\mathrm{HCO}^{+}\right)$and $R_{\mathrm{D}}\left(\mathrm{N}_{2} \mathrm{H}^{+}\right) \equiv$ $N\left(\mathrm{~N}_{2} \mathrm{D}^{+}\right) / N\left(\mathrm{~N}_{2} \mathrm{H}^{+}\right)$. The values of $R_{\mathrm{D}}\left(\mathrm{HCO}^{+}\right)$and $R_{\mathrm{D}}\left(\mathrm{N}_{2} \mathrm{H}^{+}\right)$ are in the range $0.0002 \pm 0.0001-0.014 \pm 0.003$ and $0.002 \pm$ $0.001-0.028 \pm 0.005$, respectively; the error in $R_{\mathrm{D}}$ was derived from the errors in the corresponding column densities (see Cols. (3) and (4) of Table 9).

\subsection{Ionisation degree}

We have derived fractional abundances of different ionic species and their different isotopologues. In principle, a lower limit to the ionisation degree can be obtained by simply summing up these abundances (e.g., Caselli et al. 2002; Miettinenet al. 2009):

$x(\mathrm{e})>x\left(\mathrm{HCO}^{+}\right)+x\left(\mathrm{H}^{13} \mathrm{CO}^{+}\right)+x\left(\mathrm{DCO}^{+}\right)+x\left(\mathrm{~N}_{2} \mathrm{H}^{+}\right)+x\left(\mathrm{~N}_{2} \mathrm{D}^{+}\right)$.

This is based on the gas quasi-neutrality: the electron abundance equals the difference between the total abundances of the cations and the anions. The resulting values, $x(\mathrm{e})>\sum_{i} x(\text { ions })_{i}$, are listed in Col. (5) of Table 9. These lower limits are in the range $7 \times 10^{-9}-1.2 \times 10^{-7}$.

The fractional ionisation in dense molecular clouds can also be inferred by employing ion-molecule chemical reaction schemes with the observed molecular abundances. All reactions considered here and notes on the associated rate coefficients are given in Table 8.
At first, we derived another estimate for a lower limit to $x(\mathrm{e})$ through the ionisation balance determined by $\mathrm{H}_{3}^{+}, \mathrm{HCO}^{+}, \mathrm{N}_{2} \mathrm{H}^{+}$, and electrons following Qi et al. (2003). The $\mathrm{H}_{3}^{+}$and $\mathrm{N}_{2} \mathrm{H}^{+}$are mainly destroyed by $\mathrm{CO}$; at steady state, reactions 3,11 , and 15 in Table 8 lead to the following equation for the lower limit to $x$ (e) (cf. Eq. (6) in Qi et al. 2003):

$x(\mathrm{e}) \geq \frac{k_{15} x\left(\mathrm{~N}_{2} \mathrm{H}^{+}\right) x(\mathrm{CO})}{\beta_{11} x\left(\mathrm{HCO}^{+}\right)}$.

The rate coefficients $k_{15}$ and $\beta_{11}$ were adopted from the UMIST database $^{14}$ (Woodall et al. 2007). The derived values are listed in Col. (6) of Table 9. For I18151 MM2 and I18223 MM2 the $x(\mathrm{e})$ values derived from Eq. (10) are similar to those computed from Eq. (9). For I18102 MM1, G015.31 MM3, and I18182 MM2 the summed abundance of different ionic species is clearly higher (by factors $\sim 3.5-16.3$ ) than the lower limit to $x(\mathrm{e})$ resulting from Eq. (10). In the case of G015.05 MM1, on the other hand, the summed ionic abundance is about six times lower. These discrepancies are not surprising because the chemical scheme behind Eq. (10) is certainly oversimplified.

Next, we determined the degree of ionisation by utilising the abundance ratios $R_{\mathrm{D}}\left(\mathrm{HCO}^{+}\right) \equiv\left[\mathrm{DCO}^{+}\right] /\left[\mathrm{HCO}^{+}\right]$and $R_{\mathrm{H}} \equiv\left[\mathrm{HCO}^{+}\right] /[\mathrm{CO}]$. The first studies of fractional ionisation based on the above ratios were carried out more than three decades ago (e.g., Guélin et al. 1977; Watson et al. 1978; Wootten et al. 1979). A similar analysis was subsequently applied in the papers by Caselli et al. (1998), Williams et al. (1998), Bergin et al. (1999), Anderson et al. (1999), and Caselli (2002).

We note that the following analysis includes several assumptions: $i$ ) $\mathrm{HCO}^{+}$is mainly produced in the reaction between $\mathrm{H}_{3}^{+}$ and $\mathrm{CO}$ (reaction 3 in Table 8); ii) all deuteration is caused by the reaction between $\mathrm{H}_{3}^{+}$and $\mathrm{HD}$ (reaction 2); iii) the presence of atomic deuterium, which could (slightly) increase the deuteration degree, is ignored; $i v$ ) ionic species are destroyed mainly by electrons, the most important neutrals ( $\mathrm{CO}$ and $\mathrm{O}$ ), and negatively charged dust grains; $v$ ) except $\mathrm{CO}$ and $\mathrm{O}$, we neglect the contribution of some other neutral species, such as $\mathrm{N}_{2}, \mathrm{O}_{2}$, and $\mathrm{H}_{2} \mathrm{O}$, in the destruction of $\mathrm{H}_{3}^{+}$and $\mathrm{H}_{2} \mathrm{D}^{+}$; and $v i$ ) we ignore the effects of refractory metals (Anderson et al. 1999; Caselli et al. 2002). Concerning part $v$ ), the abundances of neutrals, such as $\mathrm{N}_{2}$ and $\mathrm{H}_{2} \mathrm{O}$, are poorly known and/or low. For instance, the first results from Herschel have shown that $\mathrm{H}_{2} \mathrm{O}$ abundance is relatively low in high-mass star-forming regions $\left(\sim 10^{-10}-10^{-8}\right.$; van der Tak et al. 2010; Marseille et al. 2010a; Chavarría et al. 2010). Bergin et al. (1999) varied the nitrogen abundance in their chemical model and found that it does not affect the electron abundance. Also, the destructive reaction with $\mathrm{O}_{2}$ is very slow $\left(k=9.3 \times 10^{-10} \mathrm{~cm}^{3} \mathrm{~s}^{-1}\right.$; UMIST).

By writing steady-state equations for the abundances of $\mathrm{H}_{2} \mathrm{D}^{+}$(reactions 2 and 7-10), $\mathrm{DCO}^{+}$(reactions 7 and 13-14), and $\mathrm{HCO}^{+}$(reactions 3, 7, and 11-12), it can be shown that

$R_{\mathrm{D}}\left(\mathrm{HCO}^{+}\right)=\frac{1}{3} \frac{k_{+} x(\mathrm{HD})}{k_{7} x(\mathrm{CO})+\beta_{8} x(\mathrm{e})+k_{9} x(\mathrm{O})+k_{10} x(\mathrm{~g})}$.

Solving $x(\mathrm{e})$ from the above formula yields

$x(\mathrm{e})=\frac{1}{\beta_{8}}\left[\frac{k_{+} x(\mathrm{HD})}{3 R_{\mathrm{D}}\left(\mathrm{HCO}^{+}\right)}-k_{7} x(\mathrm{CO})-k_{9} x(\mathrm{O})-k_{10} x(\mathrm{~g})\right]$.

This represents the upper limit to $x(\mathrm{e})$. We have assumed that $k_{7}=1 / 3 \times k_{3}$, because $\mathrm{H}_{2} \mathrm{D}^{+}$can transfer a proton to $\mathrm{CO}$

14 http://www.udfa.net/ 
Table 9. Depletion-, deuteration-, and ionisation parameters of the clumps.

\begin{tabular}{|c|c|c|c|c|c|c|c|}
\hline Source & $\overline{\overline{f_{\mathrm{D}}}}$ & $\overline{R_{\mathrm{D}}\left(\mathrm{HCO}^{+}\right)}$ & $\overline{R_{\mathrm{D}}\left(\mathrm{N}_{2} \mathrm{H}^{+}\right)}$ & $\begin{array}{c}\sum_{i} x(\text { ions })_{i} \\
{\left[10^{-8}\right]}\end{array}$ & $\begin{array}{c}x(\mathrm{e})_{l} \\
{\left[10^{-8}\right]}\end{array}$ & $\begin{array}{c}x(\mathrm{e})_{u} \\
{\left[10^{-5}\right]}\end{array}$ & $\begin{array}{c}\zeta_{\mathrm{H}_{2}} \\
{\left[10^{-17} \mathrm{~s}^{-1}\right]}\end{array}$ \\
\hline IRDC 18102-1800 MM1 & $0.7 \pm 0.1$ & $0.0002 \pm 0.0001$ & $0.002 \pm 0.001$ & $12.3 \pm 6.4$ & 3.5 & 29.2 & 115 \\
\hline G015.05+00.07 MM1 & $0.8 \pm 0.1$ & $0.006 \pm 0.003$ & $0.028 \pm 0.005$ & $0.9 \pm 0.3$ & 5.6 & 0.8 & 1.2 \\
\hline IRDC 18151-1208 MM2 & $1.6 \pm 0.3$ & $0.003 \pm 0.001$ & $0.010 \pm 0.002^{a}$ & $0.7 \pm 0.1$ & 0.7 & 1.8 & 5.0 \\
\hline G015.31-00.16 MM3 & $2.7 \pm 1.3$ & $0.007 \pm 0.002$ & . & $2.3 \pm 0.8$ & 0.6 & 0.7 & 5.5 \\
\hline IRDC 18182-1433 MM2 & $1.1 \pm 0.2$ & $0.014 \pm 0.003$ & . & $4.9 \pm 1.1$ & 0.3 & 0.2 & 19.7 \\
\hline IRDC 18223-1243 MM3 & $1.3 \pm 0.1$ & $0.003 \pm 0.001$ & $0.013 \pm 0.001^{a}$ & $1.8 \pm 0.5$ & 1.6 & 1.8 & 13.1 \\
\hline ISOSS J18364-0221 SMM1 & $0.6 \pm 0.1$ & $0.012 \pm 0.006$ & . & $4.1 \pm 1.3$ & $\ldots$ & 0.3 & 51.1 \\
\hline
\end{tabular}

Notes. ${ }^{(a)}$ Calculated by utilising $N\left(\mathrm{~N}_{2} \mathrm{H}^{+}\right)$from SSK08 (see Sects. 4.2 and 5.1).

producing $\mathrm{HCO}^{+}$; the rate for this is twice as high as for the channel producing $\mathrm{DCO}^{+}$. For the reaction rate of the deuteration reaction $\mathrm{H}_{3}^{+}+\mathrm{HD}\left(k_{+}\right)$we used the recent results by Hugo et al. (2009, their Table VIII). For example, at $10 \mathrm{~K}$ the deuteration of $\mathrm{H}_{3}^{+}$proceeds about 4.4 times faster according to Hugo et al. (2009) than suggested by laboratory measurements of Gerlich et al. (2002). Such a high rate was also used in the studies cited above, i.e., $k_{+}=1.5 \times 10^{-9} \mathrm{~cm}^{3} \mathrm{~s}^{-1}$ (e.g., Caselli et al. 1998). We take the HD abundance to be twice the elemental D/H-ratio, i.e., $x(\mathrm{HD})=2 \times[\mathrm{D}] /[\mathrm{H}] \sim 3 \times 10^{-5}$ (e.g., Linsky et al. 2006; Prodanović et al. 2010). Because CO is not found to be depleted in our clumps, we assume that this is also the case for atomic oxygen and use the "standard" abundance relative to $\mathrm{H}_{2}$ of $x(\mathrm{O})=3.52 \times 10^{-4}$, i.e., comparable to $x(\mathrm{CO})$ (see Caselli et al. 1998; Caselli 2002). For the grain abundance, $x(\mathrm{~g})$, we use the value $2.64 \times 10^{-12}$, which is based on the values $a=0.1 \mu \mathrm{m}, \rho_{\text {grain }}=3 \mathrm{~g} \mathrm{~cm}^{-3}$, and $R_{\mathrm{d}}=1 / 100$ (see, e.g., Eq. (15) in Pagani et al. 2009a). The derived upper limits to $x(\mathrm{e})$ are shown in Col. (7) of Table 9. The values lie in the range $\sim 2 \times 10^{-6}-\sim 3 \times 10^{-4}$, i.e., much higher than the lower limits estimated above.

By deriving a steady-state equation for the $\mathrm{H}_{3}^{+}$abundance (see reactions 1-6 in Table 8) and applying it in the corresponding equation for $\mathrm{HCO}^{+}$, it can be shown that

$$
R_{\mathrm{H}}=\frac{\left[\zeta_{\mathrm{H}_{2}} / n\left(\mathrm{H}_{2}\right)\right] k_{3}}{\left[\beta_{4} x(\mathrm{e})+k_{3} x(\mathrm{CO})+k_{5} x(\mathrm{O})+k_{6} x(\mathrm{~g})\right]\left[\beta_{11} x(\mathrm{e})+k_{12} x(\mathrm{~g})\right]} .
$$

After $\mathrm{H}_{2}^{+}$has formed via cosmic-ray ionisation of $\mathrm{H}_{2}$ (reaction 1), it quickly reacts with $\mathrm{H}_{2}$ to form $\mathrm{H}_{3}^{+}$ (Solomon \& Werner 1971). Thus the $\mathrm{H}_{3}^{+}$abundance is governed by the rate $\zeta_{\mathrm{H}_{2}}$. When the fractional ionisation in the source is determined, Eq. (13) can be used to infer the cosmic-ray ionisation rate of $\mathrm{H}_{2}$. To calculate $\zeta_{\mathrm{H}_{2}}$, for each source we adopted as $x(\mathrm{e})$ the summed abundance of ionic species. Using the derived upper limits to $x(\mathrm{e})$ would result in unrealistically high values of $\zeta_{\mathrm{H}_{2}}$. This suggests that the upper $x(\mathrm{e})$ limits are clearly higher than the true values. The obtained results are shown in the last column of Table 9 . In most cases, the $\zeta_{\mathrm{H}_{2}}$ values lie in the range $\sim 1 \times 10^{-17}-5 \times 10^{-16} \mathrm{~s}^{-1}$. However, towards I18102 MM1 we obtain a very high rate of $\sim 1 \times 10^{-15} \mathrm{~s}^{-1}$. Because $\mathrm{I} 18102$ MM1 is associated with a bright MIR point-source and shows no signs of $\mathrm{CO}$ depletion, it is probably a rapidly evolving source. Therefore, the steady-state assumption used in the above analysis may be invalid for I18102 MM1.

\section{Discussion}

In the following subsections we discuss our results and compare them with the results from previous studies.

\subsection{Molecular column densities and abundances}

Some of the molecular column densities and fractional abundances derived here have been determined in previous studies. Assuming LTE conditions and optically thin emission, SSK08 estimated $\mathrm{N}_{2} \mathrm{H}^{+}$column densities from the $J=1-0$ transition for I18102 MM1, G015.05 MM1, and G015.31 MM3, which are clearly lower than our values (by factors $\sim 2-6$ ). Sakai et al. (2008) assumed that $T_{\text {ex }}\left(\mathrm{N}_{2} \mathrm{H}^{+}\right)=T_{\text {rot }}\left(\mathrm{NH}_{3}\right)$ even though the $T_{\text {ex }}\left(\mathrm{N}_{2} \mathrm{H}^{+}\right)$values are considerably lower than $T_{\text {rot }}\left(\mathrm{NH}_{3}\right)$ (their Table 4). On the other hand, $T_{\mathrm{ex}}\left[\mathrm{N}_{2} \mathrm{H}^{+}(1-0)\right]$ from SSK08 are very similar to those from our RADEX simulations for the $J=$ 3-2 transition. Also, the line optical thicknesses SSK08 list in their Table 4 indicate optically thick emission $(\tau \sim 2-11)$. This can explain the discrepancy in the derived column densities.

Marseille et al. (2008) modelled the $\mathrm{N}_{2} \mathrm{H}^{+}, \mathrm{HCO}^{+}$, and $\mathrm{H}^{13} \mathrm{CO}^{+}$emission of I18151 MM2, and obtained abundances that are comparable within the errors to those we have derived. This strengthens the reliability of our $\mathrm{HCO}^{+}$abundances derived from $\mathrm{H}^{13} \mathrm{CO}^{+}$by utilising the $\left[{ }^{12} \mathrm{C}\right] /\left[{ }^{13} \mathrm{C}\right]$ abundance ratio.

Sakai et al. (2010, hereafter SSH10) derived $\mathrm{H}^{13} \mathrm{CO}^{+}$column densities from the $J=1-0$ transition for I18102 MM1, I18151 MM2, and I18223 MM3. Their results are otherwise similar to ours except that for I18102 MM1 the column densities differ by a factor of $\sim 4$. Sakai et al. (2010) assumed optically thin emission and that $T_{\mathrm{ex}}\left(\mathrm{H}^{13} \mathrm{CO}^{+}\right)=T_{\text {rot }}\left(\mathrm{CH}_{3} \mathrm{OH}\right) \pm 5$ $\mathrm{K}=16.7 \pm 5 \mathrm{~K} \sim 3.6 \times T_{\mathrm{ex}}\left[\mathrm{H}^{13} \mathrm{CO}^{+}(3-2)\right]$. These authors also derived $\mathrm{SiO}$ column densities from the $J=2-1$ transition for the above three sources. We have obtained 36-86 times higher column densities for these sources from the $J=6-5$ transition through RADEX calculations. The spatial resolution of $\mathrm{SiO}$ observations by $\mathrm{SSH} 10$ was $18^{\prime \prime}$, and as $T_{\mathrm{ex}}(\mathrm{SiO})$ they used $T_{\text {rot }}\left(\mathrm{CH}_{3} \mathrm{OH}\right) \pm 5 \mathrm{~K}$, or assumed that $T_{\text {ex }}(\mathrm{SiO})=20 \mathrm{~K}$, which are over four times higher than $T_{\mathrm{ex}}[\mathrm{SiO}(6-5)]$ derived here. For comparison, we performed the LTE modelling of $\mathrm{SiO}(6-5)$ lines in Weeds, and found that column densities comparable to those obtained from RADEX are needed to explain the observed line intensities. Of course, similarly to SSH10, our RADEX and Weeds analyses of a single $\mathrm{SiO}$ transition include several (uncertain) assumptions. The large discrepancy in the derived $N(\mathrm{SiO})$ values could also be due to the fact that the $J=2-1$ and $J=6-5$ lines, which have completely different upper-state energies $(6.3 \mathrm{~K}$ and $43.8 \mathrm{~K}$, respectively), originate in different parts within a clump.

In Fig. 8 we show stock charts of the derived molecular column densities and fractional abundances. For comparison, we plot column densities and abundances from several previous studies. The quoted reference studies deal with IRDCs (Ragan et al. 2006; SSK08; Gibson et al. 2009; Beuther \& Henning 2009; Chen et al. 2010; SSH10; Vasyunina et al. 2011), high-mass young stellar objects (HMYSOs; Fontani et al. 2006; Thomas \& Fuller 2008), and low-mass starless and protostellar 


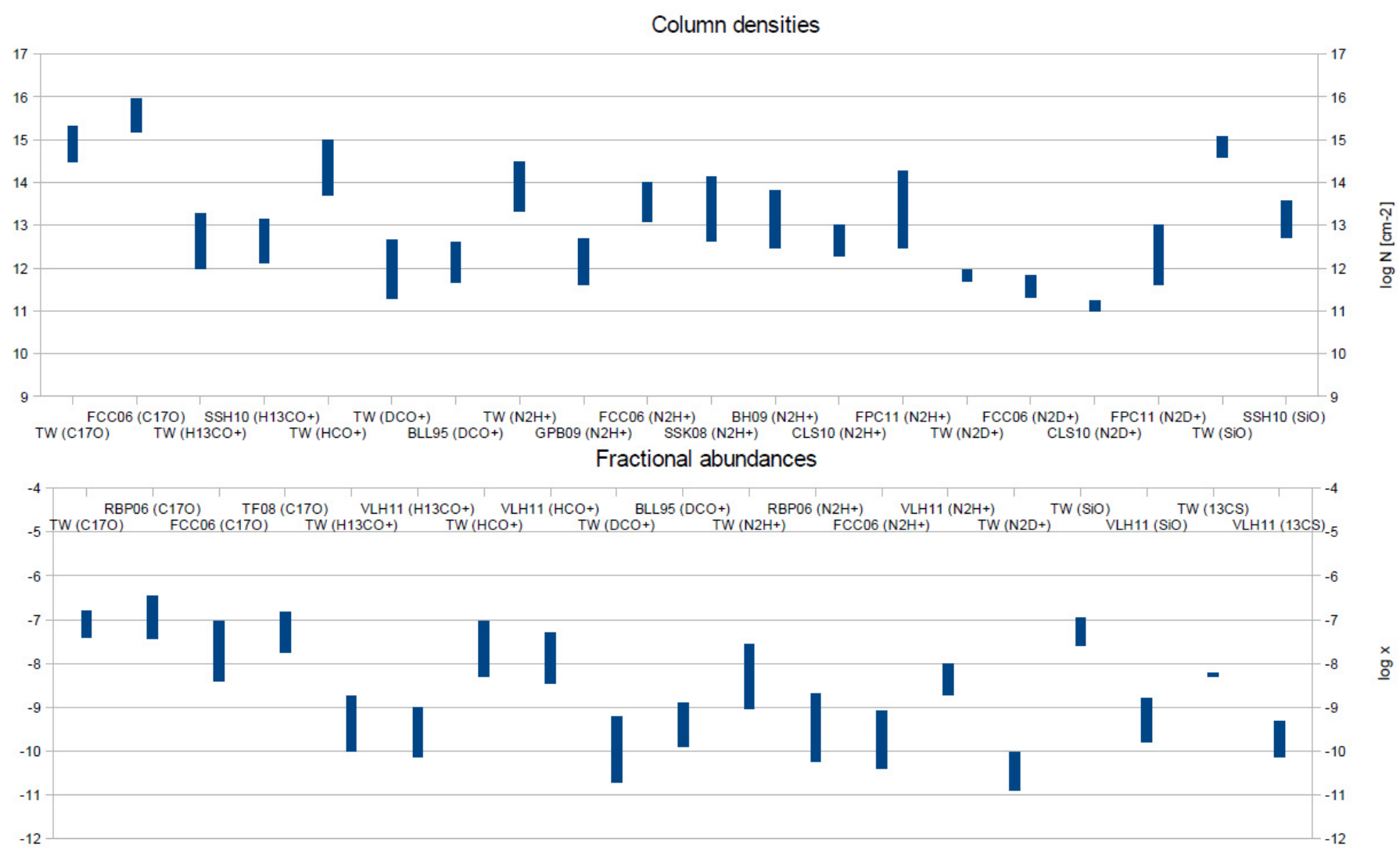

Fig. 8. Range of molecular column densities (upper panel) and fractional abundances (lower panel) for our sources as compared with similar samples of other studies. The $y$-axes are shown on a logarithmic scale. The abbreviation TW refers to this work. The references are coded as follows: FCC06 = Fontani et al. (2006), SSH10 = Sakai et al. (2010), BLL95 = Butner et al. (1995), GPB09 = Gibson et al. 2009 , SSK08 = Sakai et al. (2008), BH09 = Beuther \& Henning (2009), CLS10 = Chen et al. (2010), FPC11 = Fontani et al. (2011), RBP06 = Ragan et al. (2006), TF08 $=$ Thomas \& Fuller (2008), VLH11 = Vasyunina et al. (2011). The ${ }^{13} \mathrm{CS}$ abundance of $\sim 6.2 \times 10^{-9}$ we derived towards I18102 MM1 is shown by a slightly stretched bar for clarity.

cores (Butner et al. 1995). The latter study was included because we found no other reported $\mathrm{DCO}^{+}$surveys towards IRDCs or HMYSOs. We also plot values from Fontani et al. (2011), who studied a sample of 27 high-mass cores (including starless cores, HMYSOs, and UC HII regions). The $\mathrm{C}^{18} \mathrm{O}$ abundances from Ragan et al. (2006) were converted into $x\left(\mathrm{C}^{17} \mathrm{O}\right)$ by using the abundance ratio $\left[{ }^{18} \mathrm{O}\right] /\left[{ }^{17} \mathrm{O}\right]=3.52$.

As can be seen in Fig. 8, the column densities and abundances we have derived are generally comparable to those observed in other sources. The most evident difference is the high $\mathrm{SiO}$ and ${ }^{13} \mathrm{CS}$ column densities and abundances we have obtained compared to the IRDCs studied by SSH10 and Vasyunina et al. (2011).

Sakai et al. (2010) found that, in general, the $N(\mathrm{SiO}) / N\left(\mathrm{H}^{13} \mathrm{CO}^{+}\right)$ratio is higher for the MSX dark sources than for the sources associated with MIR emission, and suggested that this ratio represents the fraction of the shocked gas. For the sources I18102 MM1, I18151 MM2, and I18223 MM3, SSH10 found the ratios $N(\mathrm{SiO}) / N\left(\mathrm{H}^{13} \mathrm{CO}^{+}\right)=2.9_{-1.2}^{+1.9}$, $2.4_{-0.9}^{+1.5}$, and $1.6_{-0.6}^{+1.0}$, respectively. Using our column densities, these ratios become very different: $57.1 \pm 46.8,220 \pm 74.4$, and $80 \pm 20$, respectively. The latter values agree better with the general trend found by SSH10 for a sample of 20 sources. These authors suggested that the $\mathrm{SiO}$ emission from the MIR dark objects originates in newly formed shocks, whereas the $\mathrm{SiO}$ emission from more evolved, MIR bright objects could originate in gas shocked earlier in time. This is supported by the fact that we see the narrowest SiO line towards I18102 MM1, presumably the most evolved source in our sample with detected $\mathrm{SiO}$ emission (see Sect. B.1 of Appendix B). Follow-up studies of extensive source samples are needed to test the statistical significance of the hypothesis by SSH10.

\subsection{Depletion and deuteration}

The $\mathrm{CO}$ depletion factors derived in this work are small, only in the range $\sim 0.6-2.7$. Thus, $\mathrm{CO}$ molecules do not appear to be significantly depleted, if at all, in the studied clumps. This conforms to the fact that the clumps show signs of star-formation activity, such as outflows, which presumably release $\mathrm{CO}$ from the dust grains back into the gas phase (Appendix B). The presence of outflow-shocked gas is revealed by the $\mathrm{SiO}$ line wings (particularly towards I18151 MM2) and very high $\mathrm{SiO}$ abundances found in the present work. Also, the temperature in the envelopes of I18102 MM1 and I18151 MM2 is, likely owing to heating by embedded YSOs, slightly higher than the $\mathrm{CO}$ sublimation temperature of $20 \mathrm{~K}$ (e.g., Aikawa et al. 2008). Moreover, relatively high cosmic-ray ionisation rates towards the clumps were found. Thus, the cosmic-ray impulsive heating could also play a role in returning CO into the gas phase (Hasegawa \& Herbst 1993).

However, the derived $\mathrm{CO}$ depletion factors represent only the average values along the line of sight within the 27 '. 8 beam. Because the studied clumps lie at kiloparsec distances from us, the observations could be "contaminated" by non-depleted gas 
along the line of sight (Fontani et al. 2006). It is also uncertain whether the "canonical" CO abundance used to calculate $f_{\mathrm{D}}$ is exactly correct. Its value is known to vary by a factor of $\sim 2$ between different star-forming regions (Lacy et al. 1994). Finally, the $\mathrm{H}_{2}$ column densities derived from dust emission suffer from the uncertainty of dust opacity, which may be a factor of 2-3. These uncertainties are likely to explain the unsual values of $f_{\mathrm{D}}<1$.

For comparison, Fontani et al. (2006) determined the amount of $\mathrm{CO}$ depletion in HMYSOs. They found $\mathrm{CO}$ depletion factors in the range $0.4-35.8$ with a median value of 3.2 . Thomas \& Fuller (2008) found depletion factors $\lesssim 10$ for their sample of HMYSOs and deduced that the sources are young, a few times $10^{5} \mathrm{yr}$.

At high angular resolution it is possible to identify more $\mathrm{CO}$ depleted regions within the clumps. Zhang et al. (2009) studied two massive clumps in the IRDC G28.34+0.06 at $\sim 1^{\prime \prime}$ resolution with the SMA. They found CO depletion factors up to $\sim 10^{2}-10^{3}$ towards the two clumps, where the highest values were found in the cores within one of the clumps. Similarly, with the $\sim 3^{\prime \prime}$ resolution PdBI observations, Beuther \& Henning (2009) found a high CS depletion factor of $\sim 100$ in IRDC 19175-4.

The degrees of deuteration in our clumps are $\sim 0.0002-0.014$ in $\mathrm{HCO}^{+}$and $\sim 0.002-0.028$ in $\mathrm{N}_{2} \mathrm{H}^{+}$. Compared to the average cosmic $\mathrm{D} / \mathrm{H}$ ratio of $\sim 1.5 \times 10^{-5}$ (Sect. 4.4), the derived values are $\sim 10^{1}-10^{3}$ times higher. Relatively low deuteration degrees in some of our sources are consistent with the oberved low $\mathrm{CO}$ depletion factors. $\mathrm{CO}$ is the main destroyer of $\mathrm{H}_{3}^{+}$and $\mathrm{H}_{2} \mathrm{D}^{+}$, and thus its presence can lower the deuterium fractionation (e.g., Caselli et al. 2008). For example, Swift (2009) estimated the fractional abundance of ortho$\mathrm{H}_{2} \mathrm{D}^{+}$in two IRDCs of only $\sim 3-5 \times 10^{-13}$, which is up to three orders of magnitude lower than found in low-mass cores (e.g., Caselli et al. 2008; Friesen et al. 2010). From a theoretical point of view, if there are no depletion gradients in the source, one would expect to see the equality $R_{\mathrm{D}}\left(\mathrm{HCO}^{+}\right)=$ $R_{\mathrm{D}}\left(\mathrm{N}_{2} \mathrm{H}^{+}\right)$(Rodgers \& Charnley 2001b). That we found somewhat lower values for $R_{\mathrm{D}}\left(\mathrm{HCO}^{+}\right)$than $R_{\mathrm{D}}\left(\mathrm{N}_{2} \mathrm{H}^{+}\right)$could be related to differential depletion of molecules, and radial density gradients (Caselli et al. 2002). For high-mass star-forming clumps, the density profile is found to be of the form $n(r) \propto$ $r^{-(1.6 \pm 0.5)}$ (e.g., Beuther et al. 2002a). CO is the parent species of $\mathrm{HCO}^{+}$, but it destroys the $\mathrm{N}_{2} \mathrm{H}^{+}$molecules. Deuteration proceeds most efficiently in regions where $\mathrm{CO}$ is mostly frozen onto dust grains. In the warmer envelope layers, where $\mathrm{CO}$ is not depleted, $\mathrm{HCO}^{+}$can have a relatively high abundance, i.e., a lower value of $R_{\mathrm{D}}\left(\mathrm{HCO}^{+}\right)$compared to that of $\mathrm{N}_{2} \mathrm{H}^{+}$ (Emprechtinger et al. 2009). In general, the amount of molecular deuteration is expected to decrease during protostellar evolution because of internal heating of the surrounding envelope (e.g., Emprechtinger et al. 2009). Indeed, there is a decreasing trend in $R_{\mathrm{D}}\left(\mathrm{HCO}^{+}\right)$with the gas kinetic temperature as shown in Fig. 9. When the temperature becomes $\gtrsim 20 \mathrm{~K}$, reaction 2 in Table 8 can proceed in both directions, and thus the destruction rate of $\mathrm{H}_{2} \mathrm{D}^{+}$ increases. Consequently, other deuterated molecules that form via reactions with $\mathrm{H}_{2} \mathrm{D}^{+}$start to decrease in abundance.

For comparison, Fontani et al. (2006) determined $R_{\mathrm{D}}\left(\mathrm{N}_{2} \mathrm{H}^{+}\right)$ in HMYSOs and found the values in the range $\lesssim 0.004-0.02$, with an average value of $\sim 0.015$. Roberts \& Millar (2007) derived the upper limits to $R_{\mathrm{D}}\left(\mathrm{N}_{2} \mathrm{H}^{+}\right)$of $<0.002$ and $<0.006$ for the hot cores G34.26 and G75.78, respectively. Our source I18102 MM1 appears similar in this regard. Chen et al. (2010) found that $R_{\mathrm{D}}\left(\mathrm{N}_{2} \mathrm{H}^{+}\right)=0.017-0.052$ in three cores within the IRDC $\mathrm{G} 28.34+0.06$. Moreover, they found that $R_{\mathrm{D}}\left(\mathrm{N}_{2} \mathrm{H}^{+}\right)$is lower

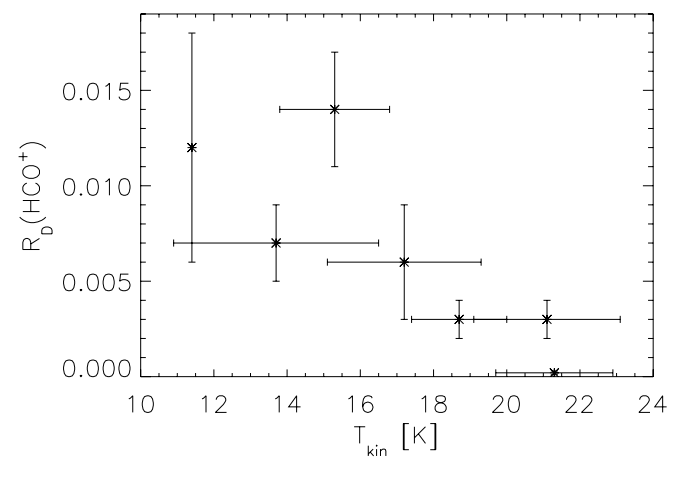

Fig. 9. $N\left(\mathrm{DCO}^{+}\right) / N\left(\mathrm{HCO}^{+}\right)$ratio versus gas kinetic temperature. As $T_{\text {kin }}$ increases, the degree of deuterium fractionation decreases.

in the more evolved stages of protostellar evolution. Recently, Fontani et al. (2011) found values of $R_{\mathrm{D}}\left(\mathrm{N}_{2} \mathrm{H}^{+}\right)=0.012-0.7$, $0.017-\leq 0.4$, and $0.017-0.08$ for their sample of high-mass starless cores, HMYSOs, and UC HII regions, respectively. The above results are quite similar to our findings. Another study of deuteration in IRDCs is that by Pillai et al. (2007), who derived $\mathrm{NH}_{2} \mathrm{D} / \mathrm{NH}_{3}$ column density ratios in the range $0.005-0.386$ for their sample of IRDC clumps. More recently, Pillai et al. (2011) found $\left[\mathrm{NH}_{2} \mathrm{D} / \mathrm{NH}_{3}\right]$ ratios in the range $0.06-0.37$ towards IRDCs. The $\left[\mathrm{NH}_{2} \mathrm{D} / \mathrm{NH}_{3}\right]$ ratios obtained by Pillai et al. are even higher than those observed in low-mass dense cores (see Pillai et al. 2011, and references therein).

Again, high-resolution interferometric observations could reveal substructures within the clumps with a higher degree of deuteration. For example, Fontani et al. (2006) found an average value $R_{\mathrm{D}}\left(\mathrm{N}_{2} \mathrm{H}^{+}\right) \sim 0.01$ towards the high-mass starforming region IRAS $05345+3157$, but at high angular resolution $\left(\sim 3^{\prime \prime}\right)$ they resolved it into two $\mathrm{N}_{2} \mathrm{D}^{+}$condensations each with ten times higher deuteration degree $\left[R_{\mathrm{D}}\left(\mathrm{N}_{2} \mathrm{H}^{+}\right)=\right.$ 0.11; Fontani et al. 2008]. These high values are comparable to those found in low-mass starless cores [Crapsi et al. 2005; $\left.R_{\mathrm{D}}\left(\mathrm{N}_{2} \mathrm{H}^{+}\right)<0.02-0.44\right]$ and Class 0 protostellar envelopes [Emprechtinger et al. 2009; $\left.R_{\mathrm{D}}\left(\mathrm{N}_{2} \mathrm{H}^{+}\right)<0.029-0.271\right]$. For their sample of low-mass dense cores, Caselli et al. (1998) found $R_{\mathrm{D}}\left(\mathrm{HCO}^{+}\right)$to lie in the range $0.025-0.07$. For a sample of more massive cores, Bergin et al. (1999) found that most sources have values of $R_{\mathrm{D}}\left(\mathrm{HCO}^{+}\right)<0.035$. This trend conforms to our results towards more massive clumps. It is possible that the cold phase during which the deuterium fractionation takes place is so short for HMYSOs, that very high deuteration degrees are not reached (Roberts \& Millar 2007). On the other hand, the high $\left[\mathrm{NH}_{2} \mathrm{D} / \mathrm{NH}_{3}\right]$ ratios found by Pillai et al. $(2007,2011)$ could indicate a different production mechanism of deuterated ammonia compared to those of $\mathrm{N}_{2} \mathrm{H}^{+}$and $\mathrm{HCO}^{+}$, which are both purely produced in the gas phase.

One caveat to the deuteration analysis is that the FWHM linewidths of the detected $\mathrm{N}_{2} \mathrm{H}^{+}$transitions are significantly broader than those of $\mathrm{N}_{2} \mathrm{D}^{+}$[Table 4, Col. (4)]. Similarly, the linewidths of $\mathrm{H}^{13} \mathrm{CO}^{+}(3-2)$ and $\mathrm{DCO}^{+}(3-2)$ are quite different compared to each other towards some of the sources. The above transition pairs have similar critical densities, and the lines were observed at comparable spatial resolutions. Therefore, the difference between the linewidths suggests that the corresponding transitions may not be tracing the same gas component. In this 
picture, the calculated deuteration degrees should only be interpreted as an average value along the line of sight.

\subsection{Ionisation}

We have calculated the average ionisation degree along the line of sight towards the clumps by utilising three different methods. The summed abundances of the ionic species yield lower limits to $x(\mathrm{e})$ in the range $\sim 0.7-12.3 \times 10^{-8}$. A very simple chemical scheme including $\mathrm{HCO}^{+}$and $\mathrm{N}_{2} \mathrm{H}^{+}$yielded another estimates for the lower limits, about $0.3-5.6 \times 10^{-8}$. Thirdly, we used the $R_{\mathrm{D}}\left(\mathrm{HCO}^{+}\right)$values to derive an upper limit to fractional ionisation, and found the range $x(\mathrm{e})=0.2-29.2 \times 10^{-5}$. To our knowledge, these are the first reported estimates of the ionisation degree in IRDCs made so far. As discussed below, the derived lower limits are likely to be more realistic estimates of $x(\mathrm{e})$, whereas the upper limits appear to be very high. The $x(\mathrm{e})$ values should be taken as rough estimates because of the simplicity of the analytical model we used. For example, our analysis is based on chemical equilibrium, which may not be valid (Caselli et al. 1998; Lintott \& Rawlings 2006). Other factors that cause uncertainties are the possible variation of $\mathrm{CO}$ depletion and fractional molecular abundances along the line of sight. Electron abundance strongly depends on the depletion of CO because $\mathrm{CO}$ destroys ionic species such as $\mathrm{H}_{3}^{+}$and $\mathrm{N}_{2} \mathrm{H}^{+}$. In this regard, one could think that the lower limit to $x(\mathrm{e})$ derived by summing up the abundances of ionic species is the most reliable one. However, there is also a possibility that the line emission from different molecules originate in different layers of the clump. Indeed, the different linewidths between $\mathrm{N}_{2} \mathrm{H}^{+}(3-2)$ and $\mathrm{N}_{2} \mathrm{D}^{+}(3-2)$ point towards this possibility (Sect. 5.2). In this case, the physical meaning of the summed abundance of ionic species is unclear. Furthermore, that we have no information about the abundances of some important molecular ions, such as $\mathrm{H}_{3}^{+}$and $\mathrm{H}_{3} \mathrm{O}^{+}$, nor the metal ions, hampers the determination of $x(\mathrm{e})$. Metal ions play an important role in the ionisation balance in the outer layers of dense clouds (Caselli 2002). That the sources show low degrees of deuteration conforms to the fairly high ionisation levels of gas. However, detailed chemical modelling is needed to perform a more accurate analysis of the ionisation degree.

The cosmic-ray ionisation rates of $\mathrm{H}_{2}$ we found are in the range $\zeta_{\mathrm{H}_{2}} \sim 1 \times 10^{-17}-1 \times 10^{-15} \mathrm{~s}^{-1}$. Six of the seven sources have estimated values between $\sim 1 \times 10^{-17}$ and $\sim 5 \times 10^{-16} \mathrm{~s}^{-1}$, but the highest rate of $\sim 10^{-15} \mathrm{~s}^{-1}$ is seen towards I18102 MM1. $\mathrm{W}$ emphasise that the $\zeta_{\mathrm{H}_{2}}$ values were derived from the lower limits to $x(\mathrm{e})$. However, as discussed above, we believe that these estimates are more accurate than those using the upper limits to $x(\mathrm{e})$. Also, the applicability of the volume-averaged $\mathrm{H}_{2}$ number densities used to compute $\zeta_{\mathrm{H}_{2}}$ is uncertain because all detected molecular-ion transitions have a much higher critical density (Hezareh et al. 2008). The standard relation between the electron abundance and the $\mathrm{H}_{2}$ number density is $x(\mathrm{e}) \sim$ $1.3 \times 10^{-5} n\left(\mathrm{H}_{2}\right)^{-1 / 2}$ (McKee 1989). This is based on the pure cosmic-ray ionisation with the rate $1.3 \times 10^{-17} \mathrm{~s}^{-1}$ and includes no depletion of heavy elements. By using the densities derived in the present work, $1.1-18.5 \times 10^{4} \mathrm{~cm}^{-3}$, the standard relation yields the values $x(\mathrm{e}) \sim 3-12 \times 10^{-8}$. These are comparable to the estimated lower limits to $x(\mathrm{e})$.

How do our results compare to those found in other sources? Caselli et al. (1998) determined $x(\mathrm{e})$ in a sample of 24 low-mass cores consisting of both starless and protostellar objects. Their analysis was based on observations of $\mathrm{CO}, \mathrm{HCO}^{+}$, and $\mathrm{DCO}^{+}$, and the resulting values were in the range $10^{-8}$ to $10^{-6}$. They argued that the variation in $x(\mathrm{e})$ among the sources is caused by variations in metal abundance and $\zeta_{\mathrm{H}_{2}}$. Williams et al. (1998) used observations of $\mathrm{C}^{18} \mathrm{O}, \mathrm{H}^{13} \mathrm{CO}^{+}$, and $\mathrm{DCO}^{+}$to determine the values $10^{-7.5} \lesssim x(\mathrm{e}) \lesssim 10^{-6.5}$ in a similar sample of low-mass cores as Caselli et al. (1998), but they used a slightly different analysis. Applying the same analysis as Williams et al. (1998), Bergin et al. (1999) found the ionisation levels of $10^{-7.3} \lesssim x(\mathrm{e}) \lesssim$ $10^{-6.9}$ towards more massive cores (in Orion) than to those studied by Williams et al. The most massive sources were found to have the lowest electron abundances, $x(\mathrm{e})<10^{-8}$. This conforms to the results by de Boisanger et al. (1996), who found that $x(\mathrm{e}) \sim 10^{-8}$ in the massive star-forming regions NGC 2264 IRS1 and W3 IRS5. Hezareh et al. (2008) studied the high-mass star-forming region DR2 $1(\mathrm{OH})$ in Cygnus $\mathrm{X}$, and found that $x(\mathrm{e})=3.2 \times 10^{-8}$. These are consistent with our lower limits to $x(\mathrm{e})$ in massive clumps within IRDCs. The very high upper limits to $x(\mathrm{e})$ we obtained, particularly that in I18102 MM1 $\left(\sim 10^{-4}\right)$, resemble those found in PDRs, where carbon can provide most of the charge and $x(\mathrm{e}) \simeq x\left(\mathrm{C}^{+}\right)($Goicoechea et al. 2009).

Observational results presented in the literature suggest relatively large environmental variations in $\zeta_{\mathrm{H}_{2}}{ }^{15}$. van der Tak \& van Dishoeck (2000) used $\mathrm{H}_{3}^{+}$and $\mathrm{H}^{13} \mathrm{CO}^{+}$observations to constrain the ionisation rate towards HMYSO envelopes, and obtained the best-fit value $\zeta_{\mathrm{H}_{2}}=(2.6 \pm 1.8) \times 10^{-17} \mathrm{~s}^{-1}$. We found comparable rates towards three of our sources. Caselli et al. (1998) inferred the values of $\zeta_{\mathrm{H}_{2}}$ spanning a range of two orders of magnitude between $10^{-18}-10^{-16} \mathrm{~s}^{-1}$ in low-mass cores. Some of this variation could be caused by different cosmicray flux in the source regions. Williams et al. (1998) deduced a mean value of $\zeta_{\mathrm{H}_{2}}=5 \times 10^{-17} \mathrm{~s}^{-1}$. On the other hand, $\mathrm{H}_{3}^{+}$observations towards the diffure ISM suggest higher values in the range $\zeta_{\mathrm{H}_{2}} \sim 0.5-1.2 \times 10^{-15} \mathrm{~s}^{-1}$ (McCall et al. 2003; Indriolo et al. 2007). The ionisation rate towards the Galactic Centre clouds is also found to be very high, $\zeta_{\mathrm{H}_{2}}>10^{-15} \mathrm{~s}^{-1}$ up to $\sim 10^{-13} \mathrm{~s}^{-1}$ (e.g., Oka et al. 2005; Yusef-Zadeh et al. 2007; Goto et al. 2008). However, these high rates can explain the high temperatures of $\sim 70-100 \mathrm{~K}$ in the Galactic Centre clouds, whereas the dense YSO envelopes are typically much colder. van der Tak et al. (2006) estimated that $\zeta_{\mathrm{H}_{2}} \sim 4 \times 10^{-16} \mathrm{~s}^{-1}$ in the Sgr B2 envelope, whereas Hezareh et al. (2008) found a lower value of $\zeta_{\mathrm{H}_{2}}=3.1 \times 10^{-18} \mathrm{~s}^{-1}$ towards DR21 $(\mathrm{OH})$. Observations of the Horsehead Nebula by Goicoechea et al. (2009) could only be reproduced with $\zeta_{\mathrm{H}_{2}}=7.7 \pm 4.6 \times 10^{-17} \mathrm{~s}^{-1}$. Despite all the uncertainty factors in $\zeta_{\mathrm{H}_{2}}$, it seems possible that some of our clumps could be exposed to somewhat higher cosmic-ray flux, but the observed clump temperatures, $\simeq 20 \mathrm{~K}$, suggest that $\zeta_{\mathrm{H}_{2}}$ is $\sim 10^{-17}-10^{-16} \mathrm{~s}^{-1}$ (see Bergin et al. 1999).

Because (most of) our sources are associated with highmass star formation, which could increase the local UV radiation field, the role played by photoionisation should also be considered. Photoionisation is expected to become negligible when the extinction is $A_{v} \gtrsim 4 \mathrm{mag}$ (McKee 1989). In units of $N\left(\mathrm{H}_{2}\right)$ this corresponds to about $\gtrsim 4.9 \times 10^{21} \mathrm{~cm}^{-2}$ (Vuong et al. 2003). Therefore, the ionisation in the studied clumps is very likely dominated by cosmic ray particles and the ambient UV radiation field is not important in this regard. If the clumps are inhomogeneous, however, external UV radiation could penetrate more efficiently into them and enhance the ionisation level (Boissé 1990). Our sources also show high $\mathrm{HCO}^{+}$abundances $\left(\sim 5 \times 10^{-9}-9 \times 10^{-8}\right)$, whereas in the presence of significant UV radiation one would expect to observe lower values of

\footnotetext{
15 In the following, we used the relation $2.3 \zeta_{\mathrm{H}}=1.5 \zeta_{\mathrm{H}_{2}}$ to convert the ionisation rate per $\mathrm{H}$ atom, $\zeta_{\mathrm{H}}$, into $\zeta_{\mathrm{H}_{2}}$.
} 
$x\left(\mathrm{HCO}^{+}\right)$because the photoproduced electrons raise the $\mathrm{HCO}^{+}$ recombination rate (Jansen et al. 1995). On the other hand, the $\mathrm{HCO}^{+}$abundance could be enhanced by the presence of protostellar outflows where shock-generated UV radiation is present (Rawlings et al. 2000). This could explain the high $\mathrm{HCO}^{+}$abundance in I18102 MM1 (Sect. B.1).

\subsection{Coupling between the ions and neutrals}

The knowledge of the gas ionisation degree enables one to estimate the level of coupling between the neutral gas component and the pervasive magnetic field. The coupling strength can be quantified through the wave-coupling number, which is defined as the ratio of the clump radius to the MHD cutoff wavelength, $W \equiv R / \lambda_{\min }$ (Myers \& Khersonsky 1995). The wavelength $\lambda_{\min }$ represents the minimum wavelength for propagation of MHD waves, and is given by $\lambda_{\min } \equiv \pi v_{\mathrm{A}} \tau_{\text {in }}$, where $v_{\mathrm{A}}$ is the Alfvén speed, and $\tau_{\text {in }}$ is the ion-neutral collision time. When $\lambda<\lambda_{\min }$, the frequency of the wave is higher than the collision frequency between the ions and neutrals $\left(v \gg v_{\text {in }}\right)$. In this case, the neutral fluid component is decoupled from the magnetic-field dynamics. If the clump is much larger than $\lambda_{\text {min }}$, i.e., $W \gg 1$, the magnetic field is strongly coupled to the neutral component, and MHD waves can stabilise the clump.

We calculated the $W$ number by utilising Eq. (6) in Williams et al. (1998):

$$
W=\frac{x(\mathrm{e}) n\left(\mathrm{H}_{2}\right)^{1 / 2}\left\langle\sigma_{\text {in }} v\right\rangle}{\pi}\left\{\frac{5(1+p)}{4 \pi \mu m_{\mathrm{H}} G-15 \sigma_{\mathrm{T}}^{2} /\left[n\left(\mathrm{H}_{2}\right) R^{2}\right]}\right\}^{1 / 2},
$$

where $\left\langle\sigma_{\text {in }} v\right\rangle$ is the ion-neutral collision rate, $p \in[0,1]$ is a parameter describing the level of turbulence ( 0 for a minimum amount of turbulent motions, 1 for a maximum level of turbulence), $\mu$ is the mean molecular weight per particle (taken to be 2.33), $G$ is the gravitational constant, and $\sigma_{\mathrm{T}}=\sqrt{k_{\mathrm{B}} T_{\mathrm{kin}} / \mu m_{\mathrm{H}}}$ is the one-dimensional thermal velocity dispersion. Following Williams et al. (1998), we take $\left\langle\sigma_{\text {in }} v\right\rangle=1.5 \times 10^{-9} \mathrm{~cm}^{3} \mathrm{~s}^{-1}$. We used as $x(\mathrm{e})$ the summed abundance of ionic species, and consequently the values of $W$ should be taken as lower limits. We assumed that the clumps are turbulent $(p=1)$, and clump radii were taken to be the effective radii listed in Col. (7) of Table 5. Note that the latter were also used to derive the $\mathrm{H}_{2}$ number densities. The computed values of $W$ and $\lambda_{\min }$ are given in Table 10 . The derived $W$ values are in the range $\sim 2-19$, whereas the minimum wavelengths lie between 0.018 and 0.174 pc. Note that the clump radii range from 0.15 to $0.34 \mathrm{pc}$, and are mostly comparable to each other. Thus, the large variation of the $W$ values cannot (solely) be explained by different source sizes. Because $W$ represents the ratio of maximum to minimum wavelength, the maximum wavelength corresponds to the clump radius. For comparison, Bergin et al. (1999) deduced that massive cores in Orion (comparable in size to our clumps) are characterised by the value $W=20$. The MHD wave power transmission at the lowest frequency of wave propagation (i.e., the longest wavelength) rapidly drops below unity when $W \lesssim 100$ (Myers \& Lazarian 1998; their Fig. 1). Therefore, the values $W<100$ imply that the coupling is "marginal", and that MHD waves have a quite narrow band of wavelengths to propagate above $\lambda_{\min }$. However, for I18102 MM1 and J18364 SMM1 the allowed wavelengths of MHD waves could extend from the effective radii $\sim 0.2-0.3 \mathrm{pc}$ down to $\lambda_{\min } \sim 0.02-0.04 \mathrm{pc}$.
Table 10. The field-neutral coupling parameter $(W)$, MHD cutoff wavelength $\left(\lambda_{\min }\right)$, and ambipolar diffusion timescale $\left(\tau_{\mathrm{AD}}\right)$.

\begin{tabular}{lccc}
\hline \hline Source & $W$ & $\begin{array}{c}\lambda_{\min } \\
{[\mathrm{pc}]}\end{array}$ & $\begin{array}{c}\tau_{\mathrm{AD}}{ }^{a} \\
{\left[10^{5} \mathrm{yr}\right]}\end{array}$ \\
\hline IRDC 18102-1800 MM1 & 18.5 & 0.018 & $37 / 162$ \\
G015.05+00.07 MM1 & 2.1 & 0.072 & $3 / 12$ \\
IRDC 18151-1208 MM2 & 2.6 & 0.065 & $2 / 9$ \\
G015.31-00.16 MM3 & 2.2 & 0.174 & $7 / 30$ \\
IRDC 18182-1433 MM2 & 6.0 & 0.044 & $16 / 65$ \\
IRDC 18223-1243 MM3 & 4.9 & 0.037 & $5 / 24$ \\
ISOSS J18364-0221 SMM1 & 8.9 & 0.036 & $12 / 54$ \\
\hline
\end{tabular}

Notes. ${ }^{(a)}$ The first $\tau_{\mathrm{AD}}$ value was estimated from the computed value of $W$, and the second one was calculated by assuming that $\mathrm{HCO}^{+}$is the dominant ionic species (see text).

This is expected to lead to a clumpy structure of a medium (Myers \& Khersonsky 1995), just like is found to be the case in J18364 SMM1, which is fragmented into two subcores (see Sect. B.6).

Another approach to investigate the coupling of the neutral gas and the magnetic field is to determine the ratio between the ambipolar diffusion (AD) timescale and free-fall timescale, $\tau_{\mathrm{AD}} / \tau_{\mathrm{ff}}$. In the process of AD, neutrals drift quasi-statically (under their own self-gravity) relative to ions and magnetic field (Mestel \& Spitzer 1956). It can be shown that the ratio $\tau_{\mathrm{AD}} / \tau_{\mathrm{ff}}$ is approximately given by $W \sim \tau_{\mathrm{ff}} / \tau_{\text {in }}$ (see Mouschovias 1991; Williams et al. 1998). The free-fall timescales of our clumps, $\tau_{\mathrm{ff}}=\sqrt{3 \pi / 32 G\langle\rho\rangle} \sim 8 \times 10^{4}-3 \times 10^{5} \mathrm{yr}$, imply AD timescales in the range $\tau_{\mathrm{AD}} \sim W \times \tau_{\mathrm{ff}} \sim 2 \times 10^{5}-4 \times 10^{6} \mathrm{yr}$ [see the first values in Col. (4) of Table 10]. We note that $\tau_{\mathrm{AD}}$ strongly depends on the ionic composition and can be even much longer than the above rough estimates. Assuming that $\mathrm{HCO}^{+}$is the dominant ionic species, the AD timescale is given by $\tau_{\mathrm{AD}} \sim 1.3 \times 10^{14} x(\mathrm{e}) \mathrm{yr}$ (see Eq. (5) in Walmsley et al. 2004). Using again the lower limits to $x(\mathrm{e})$, we obtain timescales in the range $\tau_{\mathrm{AD}} \sim 1-16 \times 10^{6} \mathrm{yr}$, which are about four times longer than the above estimates [see the second values in Col. (4) of Table 10]. Therefore, on the scale probed by our single-dish observations, AD is not expected to be a main driver of clump evolution unless it occurs on timescales much longer than $10^{6} \mathrm{yr}$. However, the situation may be different at smaller scales where the density is higher, and therefore $x(\mathrm{e})$ is lower and $\tau_{\mathrm{AD}}$ is shorter.

\section{Summary and conclusions}

We have carried out a molecular-line study of seven massive clumps associated with IRDCs. We used APEX to observe transitions of $\mathrm{C}^{17} \mathrm{O}, \mathrm{H}^{13} \mathrm{CO}^{+}, \mathrm{DCO}^{+}, \mathrm{N}_{2} \mathrm{H}^{+}$, and $\mathrm{N}_{2} \mathrm{D}^{+}$. The principal aim of this study was to investigate the depletion, deuterium fractionation, and the degree of ionisation in the sources. Our main results are summarised as follows:

1. The CO molecules do not appear to be significantly depleted, if at all, in the observed sources. The largest $\mathrm{CO}$ depletion factor, 2.7 , is found towards G015.31 MM3, which is dark at MIR. In many sources, $\mathrm{CO}$ is likely to be released from dust grains into the gas phase owing to shock evaporation.

2. The deuteration degree in $\mathrm{HCO}^{+}$was found to range from 0.0002 to 0.014 , whereas that in $\mathrm{N}_{2} \mathrm{H}^{+}$lies in the range $0.002-0.028$. These are lower values than found in low-mass starless cores and protostellar envelopes, but still significantly higher than the cosmic D/H-ratio $\sim 10^{-5}$. The degree 
of deuteration in $\mathrm{HCO}^{+}$appears to decrease with increasing gas temperature, as expected theoretically. This likely reflects the evolutionary stage of the clump: at early stages the source is cold and as the evolutionary stage progresses the temperature rises. In the course of evolution the amount of deuterium fractionation decreases.

3. For the first time, we have estimated the level of ionisation in IRDCs. The ionisation degree is difficult to determine accurately, especially from an analytical basis like the one in the present work. However, a lower limit to electron abundance appears to be $x(\mathrm{e}) \gtrsim 10^{-8}-10^{-7}$, which is comparable to those seen in other star-forming regions. The cosmic-ray ionisation rate of $\mathrm{H}_{2}$ were estimated to vary from $\sim 10^{-17} \mathrm{~s}^{-1}$ up to $\sim 10^{-15} \mathrm{~s}^{-1}$ in one target position. Similar estimates have been found in a variety of Galactic environments.

4. Additional molecular species were detected towards some of the sources. In particular, ortho-c- $\mathrm{C}_{3} \mathrm{H}_{2}$ was detected in four sources, and $\mathrm{SiO}$ towards three sources. This is the first reported observation of $\mathrm{c}-\mathrm{C}_{3} \mathrm{H}_{2}$ towards IRDCs. The $\mathrm{SiO}$ lines showed broad linewidths indicative of outflows. Also for the first time towards IRDCs, the deuterated ethynyl, $\mathrm{C}_{2} \mathrm{D}$, was detected in I18151-1208 MM2. We estimate the $\mathrm{C}_{2} \mathrm{D} / \mathrm{C}_{2} \mathrm{H}$ column density ratio to be about 0.07 , which agrees well with the results from other sources (Appendix A).

5. The ambipolar-diffusion timescale implied by the lower limits to $x(\mathrm{e})$ is typically $\sim$ several Myr. Therefore, on the scale probed by our observations, clump evolution cannot be mainly driven by ambipolar diffusion unless it occurs on timescales much longer than $10^{6} \mathrm{yr}$.

The present single-dish study cannot follow the chemical structure of the sources down to small scales where the evolution of the massive young stellar object(s) is taking place (e.g., the case of J18364 SMM1). Follow-up high-resolution studies are needed i) to resolve whether the clumps are fragmented into smaller subunits (as is known to be the case in J18364 SMM1); ii) to examine the kinematics (infall, outflow, relative motions) of the subfragments, and, importantly, their chemical partition (such as depletion and deuteration); and iii) to locate the detected complex molecules (envelope or hot core?). In this regard, follow-up observations with ALMA seem particularly worthwhile. Finally, detailed chemical models are needed to better understand the observed molecular abundances, and how these are related to the timescale of the high-mass star-formation process.

Acknowledgements. We are indebted to the referee for his/her valuable comments, which helped significantly in making the paper more readable. We thank the staff at the APEX telescope for performing the service mode observations presented in this paper, and the ESO archive team for their help with the data products. O.M. acknowledges support from the Academy of Finland grant 132291. Many thanks to Henrik Beuther for providing us the MAMBO 1.2-mm dust continuum map of IRDC 18151-1208. We would also like to thank the people who maintain the CDMS and JPL molecular spectroscopy databases, and the Splatalogue Database for Astronomical Spectroscopy.

The present study makes use of the JCMT Archive project, which is a collaboration between the Canadian Astronomy Data Centre (CADC), Victoria, and the James Clerk Maxwell Telescope (JCMT), Hilo. This work also makes use of observations made with the Spitzer Space Telescope, which is operated by the Jet Propulsion Laboratory (JPL), California Institute of Technology under a contract with NASA. Moreover, this research has made use of NASA's Astrophysics Data System and the NASA/IPAC Infrared Science Archive, which is operated by the JPL, California Institute of Technology, under contract with the NASA

\section{References}

Aikawa, Y., Wakelam, V., Garrod, R. T., \& Herbst, E. 2008, ApJ, 674, 984 Anderson, I. M., Caselli, P., Haikala, L. K., \& Harju, J. 1999, A\&A, 347, 983 Bally, J., Aguirre, J., Battersby, C., et al. 2010, ApJ, 721, 137
Battersby, C., Bally, J., Jackson, J. M., et al. 2010, ApJ, 721, 222

Belitsky, V., Lapkin, I., Vassilev, V., et al. 2007, in Proceedings of joint 32nd International Conference on Infrared Millimeter Waves and 15th International Conference on Terahertz Electronics, September 3-7 (Cardiff, Wales, UK: City Hall), 326

Benjamin, R. A., Churchwell, E., Babler, B. L., et al. 2003, PASP, 115, 953

Bergin, E. A., Plume, R., Williams, J. P., \& Myers, P. C. 1999, ApJ, 512, 724

Beuther, H., \& Henning, T. 2009, A\&A, 503, 859 (BH09)

Beuther, H., \& Sridharan, T. K. 2007, ApJ, 668, 348

Beuther, H., \& Steinacker, J. 2007, ApJ, 656, L85

Beuther, H., Schilke, P., Menten, K. M., et al. 2002a, ApJ, 566, 945

Beuther, H., Walsh, A., Schilke, P., et al. 2002b, A\&A, 390, 289

Beuther, H., Zhang, Q., Sridharan, T. K., \& Chen, Y. 2005a, ApJ, 628, 800

Beuther, H., Schilke, P., Menten, K. M., et al. 2005b, ApJ, 633, 535

Beuther, H., Sridharan, T. K., \& Saito, M. 2005c, ApJ, 634, L185

Beuther, H., Zhang, Q., Sridharan, T. K., et al. 2006, A\&A, 454, 221

Beuther, H., Henning, T., Linz, H., et al. 2010, A\&A, 518, L78

Birkmann, S. M., Krause, O., \& Lemke, D. 2006, ApJ, 637, 380

Boissé, P. 1990, A\&A, 228, 483

Brand, J., \& Blitz, L. 1993, A\&A, 275, 67

Butner, H. M., Lada, E. A., \& Loren, R. B. 1995, ApJ, 448, 207 (BLL95)

Carey, S. J., Clark, F. O., Egan, M. P., et al. 1998, ApJ, 508, 721

Carey, S. J., Noriega-Crespo, A., Mizuno, D. R., et al. 2009, PASP, 121, 76

Caselli, P. 2002, Planet. Space Sci., 50, 1133

Caselli, P., \& Dore, L. 2005, A\&A, 433, 1145

Caselli, P., Hasegawa, T. I., \& Herbst, E. 1993, ApJ, 408, 548

Caselli, P., Walmsley, C. M., Terzieva, R., \& Herbst, E. 1998, ApJ, 499, 234

Caselli, P., Walmsley, C. M., Zucconi, A., et al. 2002, ApJ, 565, 344

Caselli, P., Vastel, C., Ceccarelli, C., et al. 2008, A\&A, 492, 703

Chambers, E. T., Jackson, J. M., Rathborne, J. M., \& Simon, R. 2009, ApJS, 181, 360

Charnley, S. B., Ehrenfreund, P., Millar, T. J., et al. 2004, MNRAS, 347, 157

Chavarría, L., Herpin, F., Jacq, T., et al. 2010, A\&A, 521, L37

Chen, H.-R., Liu, S.-Y., Su, Y.-N., \& Zhang, Q. 2010, ApJ, 713, L50 (CLS10)

Clemens, D. P. 1985, ApJ, 295, 422

Combes, F., Gerin, M., Wootten, A., et al. 1987, A\&A, 180, L13

Crapsi, A., Caselli, P., Walmsley, C. M., et al. 2005, ApJ, 619, 379

de Boisanger, C., Helmich, F. P., \& van Dishoeck, E. F. 1996, A\&A, 310, 315

Devine, K. E., Chandler, C. J., Brogan, C., et al. 2011, ApJ, 733, 44

Di Francesco, J., Johnstone, D., Kirk, H., et al. 2008, ApJS, 175, 277

Egan, M. P., Shipman, R. F., Price, S. D., et al. 1998, ApJ, 494, L199

Ellingsen, S. P. 2006, ApJ, 638, 241

Enoch, M. L., Young, K. E., Glenn, J., et al. 2006, ApJ, 638, 293

Emprechtinger, M., Caselli, P., Volgenau, N. H., et al. 2009, A\&A, 493, 89

Fallscheer, C., Beuther, H., Zhang, Q., Keto, E., \& Sridharan, T. K. 2009, A\&A, 504, 127

Fontani, F., Caselli, P., Crapsi, A., et al. 2006, A\&A, 460, 709 (FCC06)

Fontani, F., Caselli, P., Bourke, T. L., et al. 2008, A\&A, 477, L45

Fontani, F., Palau, A., Caselli, P., et al. 2011, A\&A, 529, L7 (FPC11)

Frerking, M. A., Langer, W. D., \& Wilson, R. W. 1982, ApJ, 262, 590

Friedel, D. N., Snyder, L. E., Remijan, A. J., \& Turner, B. E. 2005, ApJ, 632, L95

Friesen, R. K., Di Francesco, J., Myers, P. C., et al. 2010, ApJ, 718, 666

Fuller, G. A., Williams, S. J., \& Sridharan, T. K. 2005, A\&A, 442, 949

Furuya, R. S., Cesaroni, R., \& Shinnaga, H. 2011, A\&A, 525, A72

Garrod, R. T., Weaver, S. L. W., \& Herbst, E. 2008, ApJ, 682, 283

Gerlich, D., Herbst, E., \& Roueff, E. 2002, Planet. Space Sci., 50, 1275

Gibb, E. L., Whittet, D. C. B., Boogert, A. C. A., \& Tielens, A. G. G. M. 2004, ApJS, 151, 35

Gibson, D., Plume, R., Bergin, E., et al. 2009, ApJ, 705, 123 (GPB09)

Goddi, C., Greenhill, L. J., Humphreys, E. M. L., et al. 2009, ApJ, 691, 1254

Goicoechea, J. R., Pety, J., Gerin, M., et al. 2009, A\&A, 498, 771

Goldsmith, P. F., \& Langer, W. D. 1978, ApJ, 222, 881

Goldsmith, P. F., \& Langer, W. D. 1999, ApJ, 517, 209

Gordy, W., \& Cook, R. L. 1984, Microwave Molecular Spectroscopy (New York: Wiley)

Goto, M., Usuda, T., Nagata, T., et al. 2008, ApJ, 688, 306

Guélin, M., Langer, W. D., Snell, R. L., \& Wootten, H. A. 1977, ApJ, 217, L165

Hasegawa, T. I., \& Herbst, E. 1993, MNRAS, 261, 83

Hatchell, J., Thompson, M. A., Millar, T. J., \& MacDonald, G. H. 1998, A\&AS, 133,29

Hennemann, M., Birkmann, S. M., Krause, O., et al. 2009, ApJ, 693, 1379

Henning, T., Linz, H., Krause, O., et al. 2010, A\&A, 518, L95

Herbst, E., Giles, K., \& Smith, D. 1990, ApJ, 358, 468

Hezareh, T., Houde, M., McCoey, C., et al. 2008, ApJ, 684, 1221

Hildebrand, R. H. 1983, QJRAS, 24, 267

Hugo, E., Asvany, O., \& Schlemmer, S. 2009, J. Chem. Phys., 130, 164302

Indriolo, N., Geballe, T. R., Oka, T., \& McCall, B. J. 2007, ApJ, 671, 1736 
Jansen, D. J., van Dishoeck, E. F., Black, J. H., et al. 1995, A\&A, 302, 223 Kauffmann, J., \& Pillai, T. 2010, ApJ, 723, L7

Klein, B., Philipp, S. D., Krämer, I., et al. 2006, A\&A, 454, L29

Krause, O. 2003, Ph.D. Thesis, Univ. Heidelberg

Lacy, J. H., Knacke, R., Geballe, T. R., \& Tokunaga, A. T. 1994, ApJ, 428, L69

Ladd, E. F., Fuller, G. A., \& Deane, J. R. 1998, ApJ, 495, 871

Linsky, J. L., Draine, B. T., Moos, H. W., et al. 2006, ApJ, 647, 1106

Lintott, C. J., \& Rawlings, J. M. C. 2006, A\&A, 448, 425

López-Sepulcre, A., Walmsley, C. M., Cesaroni, R., et al. 2011, A\&A, 526, L2

McCall, B. J., Huneycutt, A. J., Saykally, R. J., et al. 2003, Nature, 422, 500

Maret, S., Hily-Blant, P., Pety, J., et al. 2011, A\&A, 526, A47

Marseille, M. G., Bontemps, S., Herpin, F., et al. 2008, A\&A, 488, 579

Marseille, M. G., van der Tak, F. F. S., Herpin, F., et al. 2010a, A\&A, 521, L32

Marseille, M. G., van der Tak, F. F. S., Herpin, F., \& Jacq, T. 2010b, A\&A, 522, A40

McKee, C. F. 1989, ApJ, 345, 782

Mestel, L., \& Spitzer, L., Jr. 1956, MNRAS, 116, 503

Miettinen, O., \& Harju, J. 2010, A\&A, 520, A102

Miettinen, O., Harju, J., Haikala, L. K., et al. 2009, A\&A, 500, 845

Miettinen, O., Harju, J., Haikala, L. K., \& Juvela, M. 2010, A\&A, 524, A91

Motte, F., \& André, P. 2001, A\&A, 365, 440

Motte, F., Bontemps, S., Schilke, P., et al. 2007, A\&A, 476, 1243

Mouschovias, T. C. 1991, ApJ, 373, 169

Müller, H. S. P., Schlöder, F., Stutzki, J., \& Winnewisser, G. 2005, J. Mol. Struct., 742,215

Müller, H. S. P., Belloche, A., Menten, K. M., et al. 2008, J. Mol. Spectr., 251, 319

Myers, P. C., \& Khersonsky, V. K. 1995, ApJ, 442, 186

Myers, P. C., \& Lazarian, A. 1998, ApJ, 507, L157

Nummelin, A., Bergman, P., Hjalmarson, A., et al. 2000, ApJS, 128, 213

Oka, T., Geballe, T. R., Goto, M., et al. 2005, ApJ, 632, 882

Ossenkopf, V., \& Henning, Th. 1994, A\&A, 291, 943

Pagani, L., Vastel, C., Hugo, E., et al. 2009a, A\&A, 494, 623

Pagani, L., Daniel, F., \& Dubernet, M.-L. 2009b, A\&A, 494, 719

Parise, B., Leurini, S., Schilke, P., et al. 2009, A\&A, 508, 737

Park, Y.-S., Panis, J.-F., Ohashi, N., et al. 2000, ApJ, 542, 344

Park, I. H., Wakelam, V., \& Herbst, E. 2006, A\&A, 449, 631

Parsons, H., Thompson, M. A., \& Chrysostomou, A. 2009, MNRAS, 399, 1506

Pérault, M., Omont, A., Simon, G., et al. 1996, A\&A, 315, L165

Pickett, H. M., Poynter, I. R. L., Cohen, E. A., et al. 1998, J. Quant. Spec. Radiat. Transf., 60, 883

Pillai, T., Wyrowski, F., Menten, K. M., \& Krügel, E. 2006a, A\&A, 447, 929

Pillai, T., Wyrowski, F., Carey, S. J. et al. 2006b, A\&A, 450, 569

Pillai, T., Wyrowski, F., Hatchell, J., et al. 2007, A\&A, 467, 207

Pillai, T., Kauffmann, J., Wyrowski, F., et al. 2011, A\&A, 530, A118

Prodanović, T., Steigman, G., \& Fields, B. D. 2010, MNRAS, 406, 1108

Qi, C., Kessler, J. E., Koerner, D. W., et al. 2003, ApJ, 597, 986

Qin, S.-L., Wu, Y., Huang, M., et al. 2010, ApJ, 711, 399

Ragan, S. E., Bergin, E. A., Plume, R., et al. 2006, ApJS, 166, 567 (RBP06)

Ragan, S. E., Bergin, E. A., \& Gutermuth, R. A. 2009, ApJ, 698, 324

Ragan, S. E., Bergin, E. A., \& Wilner, D. 2011, ApJ, 736, 163

Rathborne, J. M., Jackson, J. M., \& Simon, R. 2006, ApJ, 641, 389

Rathborne, J. M., Simon, R., \& Jackson, J. M. 2007, ApJ, 662, 1082

Rathborne, J. M., Jackson, J. M., Zhang, Q., \& Simon, R. 2008, ApJ, 689, 1141

Rathborne, J. M., Jackson, J. M., Chambers, E. T., et al. 2010, ApJ, 715, 310
Rawlings, J. M. C., Taylor, S. D., \& Williams, D. A. 2000, MNRAS, 313, 461

Reid, M. J., Menten, K. M., Zheng, X. W., et al. 2009, ApJ, 700, 137

Remijan, A. J., Markwick-Kemper, A., \& ALMA Working Group on Spectral Line Frequencies 2007, BAAS, 38, 963

Remijan, A. J., Hollis, J. M., Lovas, F. J., et al. 2008, ApJ, 675, L85

Requena-Torres, M. A., Martín-Pintado, J., Martín, S., \& Morris, M. R. 2008, ApJ, 672, 352

Roberts, H., \& Millar, T. J. 2007, A\&A, 471, 849

Rodgers, S. D., \& Charnley, S. B. 2001a, ApJ, 546, 324

Rodgers, S. D., \& Charnley, S. B. 2001b, ApJ, 553, 613

Roueff, E., Parise, B., \& Herbst, E. 2007, A\&A, 464, 245

Rygl, K. L. J., Wyrowski, F., Schuller, F., \& Menten, K. M. 2010, A\&A, 515, A42

Sakai, T., Sakai, N., Kamegai, K., et al. 2008, ApJ, 678, 1049 (SSK08)

Sakai, T., Sakai, N., Hirota, T., \& Yamamoto, S. 2010, ApJ, 714, 1658 (SSH10)

Sanhueza, P., Garay, G., Bronfman, L., et al. 2010, ApJ, 715, 18

Schmid-Burgk, J., Muders, D., Müller, H. S. P., \& Brupbacher-Gatehouse, B. 2004, A\&A, 419, 949

Schilke, P., Comito, C., Thorwirth, S., et al. 2006, A\&A, 454, L41

Schöier, F. L., van der Tak, F. F. S., van Dishoeck, E. F., \& Black, J. H. 2005, A\&A, 432, 369

Shiao, Y.-S. J., Looney, L. W., Remijan, A. J., et al. 2010, ApJ, 716, 286

Solomon, P. M., \& Werner, M. W. 1971, ApJ, 165, 41

Sridharan, T. K., Beuther, H., Schilke, P., et al. 2002, ApJ, 566, 931

Sridharan, T. K., Beuther, H., Saito, M., et al. 2005, ApJ, 634, L57

Swift, J. J. 2009, ApJ, 705, 1456

Tafalla, M., Myers, P. C., Caselli, P., \& Walmsley, C. M. 2004, A\&A, 416, 191

Teyssier, D., Hennebelle, P., and Pérault, M. 2002, A\&A, 382, 624

Thomas, H. S., \& Fuller, G. A. 2008, A\&A, 479, 751 (TF08)

Turner, B. E. 1991, ApJS, 76, 617

van der Tak, F. F. S., \& van Dishoeck, E. F. 2000, A\&A, 358, L79

van der Tak, F. F. S., Belloche, A., Schilke, P., et al. 2006, A\&A, 454, L99

van der Tak, F. F. S., Black, J. H., Schöier, F. L., et al. 2007, A\&A, 468, 627

van der Tak, F. F. S., Marseille, M. G., Herpin, F., et al. 2010, A\&A, 518, L107

Vassilev, V., Meledin, D., Lapkin, I., et al. 2008a, A\&A, 490, 1157

Vassilev, V., Henke, D., Lapkin, I., et al. 2008b, IEEE Microwave \& Wireless Components Letters, 55, 18

Vasyunina, T., Linz, H., Henning, T., et al. 2009, A\&A, 499, 149

Vasyunina, T., Linz, H., Henning, T., et al. 2011, A\&A, 527, A88 (VLH11)

Vrtilek, J. M., Gottlieb, C. A., Langer, W. D., et al. 1985, ApJ, 296, L35

Vuong, M. H., Montmerle, T., Grosso, N., et al. 2003, A\&A, 408, 581

Walmsley, C. M., Flower, D. R., \& Pineau des Forêts, G. 2004, A\&A, 418, 1035

Wang, Y., Zhang, Q., Rathborne, J. M., et al. 2006, ApJ, 651, L125

Wang, K., Zhang, Q., Wu, Y., \& Zhang, H. 2011, ApJ, 735, 64

Watson, W. D., Anicich, V. G., \& Huntress, W. T., Jr. 1976, ApJ, 205, L165

Watson, W. D., Snyder, L. E., \& Hollis, J. M. 1978, ApJ, 222, L145

Williams, J. P., Bergin, E. A., Caselli, P., et al. 1998, ApJ, 503, 689

Williams, S. J., Fuller, G. A., \& Sridharan, T. K. 2004, A\&A, 417, 115

Wilson, T. L., \& Rood, R. 1994, ARA\&A, 32, 191

Woodall, J., Agúndez, M., Markwick-Kemper, A. J., \& Millar, T. J. 2007, A\&A, 466, 1197

Wootten, A., Snell, R., \& Glassgold, A. E. 1979, ApJ, 234, 876

Yusef-Zadeh, F., Muno, M., Wardle, M., \& Lis, D. C. 2007, ApJ, 656, 847

Zhang, Q., Wang, Y., Pillai, T., \& Rathborne, J. 2009, ApJ, 696, 268

Zhang, S. B., Yang, J., Xu, Y., et al. 2011, ApJS, 193, 10

Pages 21 to 23 are available in the electronic edition of the journal at http: //wWw . aanda. org 


\section{Appendix A: Other detected transitions}

Besides the molecular species discussed in Sect. 5.1, the sources I18102 MM1, I18151 MM2, I18182 MM2, and I18223 MM3 show emission lines also from other species, some of which are complex organics. These include $o-\mathrm{c}-\mathrm{C}_{3} \mathrm{H}_{2}, \mathrm{C}_{2} \mathrm{D}, \mathrm{C}^{18} \mathrm{O}$ (seen in the image band), and possibly $\mathrm{CNCHO}$ (in the image band), $\mathrm{CH}_{3} \mathrm{NH}_{2}$ (blended with OCS), $\mathrm{CH}_{2} \mathrm{CHC}^{15} \mathrm{~N}$, and $\mathrm{CH}_{3} \mathrm{COCH}_{3}$. In this appendix we discuss each of these species (except $\mathrm{C}^{18} \mathrm{O}$ ) and their derived column densities and abundances.

Cyclopropenylidene $\left(c-\mathrm{C}_{3} \mathrm{H}_{2}\right)$. We have detected the ortho form of cyclic- $\mathrm{C}_{3} \mathrm{H}_{2}$ in four sources, and found the column densities and abundances in the range $\sim 7.0 \times 10^{12}-7.7 \times 10^{13} \mathrm{~cm}^{-2}$ and $\sim 1.9 \times 10^{-10}-1.2 \times 10^{-8}$, respectively. The $\mathrm{c}-\mathrm{C}_{3} \mathrm{H}_{2}$ molecule has been detected in several different Galactic sources, but to our knowledge, this is the first reported detection of this molecule towards massive clumps associated with IRDCs. For comparison, the beam-averaged c- $\mathrm{C}_{3} \mathrm{H}_{2}$ column densities and abundances of 4-9 $\times 10^{13} \mathrm{~cm}^{-2}$ and $3-7 \times 10^{-11}$ have been found in Sgr B2 (Turner 1991; Nummelin et al. 2000). These column densities are comparable to those in our clumps, but the abundances appear lower in Sgr B2.

The major formation channel of $\mathrm{c}-\mathrm{C}_{3} \mathrm{H}_{2}$ is $\mathrm{C}_{3} \mathrm{H}^{+}+\mathrm{H}_{2} \rightarrow$ $\mathrm{c}-\mathrm{C}_{3} \mathrm{H}_{3}^{+}$; c $-\mathrm{C}_{3} \mathrm{H}_{3}^{+}+\mathrm{e}^{-} \rightarrow \mathrm{c}-\mathrm{C}_{3} \mathrm{H}_{2}$ (e.g., Park et al. 2006). The very low rotational excitation temperatures we derived, 3.1$4.8 \mathrm{~K}$, suggest that $\mathrm{c}-\mathrm{C}_{3} \mathrm{H}_{2}$ emission mainly comes from the cool and relatively low-density envelope (Turner 1991). Also, higher electron abundance in the outer layers can promote the formation of $\mathrm{c}-\mathrm{C}_{3} \mathrm{H}_{2}$. This is consistent with the derived high fractional abundances.

Cyanoformaldehyde or formyl cyanide (CNCHO). The $\mathrm{CNCHO}$ transition is possibly seen in the image sideband towards I18102 MM1. Because of attenuated intensity, we cannot determine the column density of this molecule. Remijan et al. (2008) determined the values $N(\mathrm{CNCHO})=1-17 \times 10^{14} \mathrm{~cm}^{-2}$ and $x(\mathrm{CNCHO})=0.7-11 \times 10^{-9}$ towards Sgr B2(N). They suggested that $\mathrm{CNCHO}$ is likely formed in a neutral-radical reaction of formaldehyde $\left(\mathrm{H}_{2} \mathrm{CO}\right)$ and the cyanide $(\mathrm{CN})$ radical.

Methylamine $\left(\mathrm{CH}_{3} \mathrm{NH}_{2}\right)$. Rotational levels of $\mathrm{CH}_{3} \mathrm{NH}_{2}$ undergo the so-called $A$ - and $E$-type transitions due to the internal rotation of the $\mathrm{CH}_{3}$-group. We have a possible detection of the $E$-type $\mathrm{CH}_{3} \mathrm{NH}_{2}$ transition, and we derived the values $N\left(\mathrm{CH}_{3} \mathrm{NH}_{2}\right)=5 \times 10^{14} \mathrm{~cm}^{-2}$ and $x\left(\mathrm{CH}_{3} \mathrm{NH}_{2}\right)=5.1 \pm 0.6 \times 10^{-8}$. However, our $\mathrm{CH}_{3} \mathrm{NH}_{2}$ line is blended with OCS line, and therefore these values should be taken with caution. Beam-averaged column densities of $N\left(\mathrm{CH}_{3} \mathrm{NH}_{2}\right) \sim 1 \times 10^{14}-8 \times 10^{15} \mathrm{~cm}^{-2}$ have been found in Sgr B2 (Turner 1991; Nummelin et al. 2000). The origin of $\mathrm{CH}_{3} \mathrm{NH}_{2}$, and nitrogen-bearing organics in general, could be in the evaporation of ice mantles, indicative of a hotcore chemistry (e.g. Rodgers \& Charnley 2001a).

Carbonyl sulfide (OCS). The high- $J$ transition of OCS we have possibly detected (blended with $\mathrm{CH}_{3} \mathrm{NH}_{2}$ ) implies a column density and fractional abundance of $2.3 \pm 0.8 \times 10^{15} \mathrm{~cm}^{-2}$ and $2.4 \pm 0.9 \times 10^{-7}$, respectively. For example, Qin et al. (2010) detected OCS in the high-mass star-forming region G19.61-0.23. Assuming $T_{\text {rot }}=E_{\mathrm{u}} / k_{\mathrm{B}}$, they derived the beam-averaged column density and fractional abundance of $2.2 \pm 0.1 \times 10^{16} \mathrm{~cm}^{-2}$ and $2.7 \pm 0.1 \times 10^{-8}$, respectively. Note that Qin et al. achieved a much higher angular resolution with their SMA observations, and thus their high column density value could partly be caused by filtering out the extended envelope. Our coarser angular resolution probably causes a significant beam dilution. Sakai et al. (2010) derived OCS column densities of $1.2-5.5 \times 10^{14} \mathrm{~cm}^{-2}$ for their sample of 20 massive clumps associated with IRDCs, i.e., lower than found here towards I18102 MM1.

The OCS molecules form on grain surfaces through the addition of $\mathrm{S}$ atom to $\mathrm{CO}$, or via the $\mathrm{O}$ atom addition to $\mathrm{CS}$ (e.g., Charnley et al. 2004). Solid-state OCS has been detected towards high-mass star-forming regions by, e.g, Gibb et al. (2004). Sakai et al. (2010) suggested that OCS is released from the grains into the gas phase through protostellar shocks. This could well be the case in I18102 MM1 (Sect. B.1).

Deuterated ethynyl $\left(C_{2} D\right)$. We have made the first detection of $\mathrm{C}_{2} \mathrm{D}$ towards IRDCs. The column density and abundance estimated from the $N=3-2$ transition are $\sim 1.5 \times 10^{13} \mathrm{~cm}^{-2}$ and $4.2 \pm 0.5 \times 10^{-10}$, respectively. Sakai et al. (2010) detected the normal isotopologue $\mathrm{C}_{2} \mathrm{H}(N=1-0)$ towards I18151 MM2. The $\mathrm{C}_{2} \mathrm{H}$ column density they derived, $\sim 2.2 \times 10^{14} \mathrm{~cm}^{-2}$, together with $N\left(\mathrm{C}_{2} \mathrm{D}\right)$ derived by us, suggest a deuteration degree of $\sim 0.07$ in $\mathrm{C}_{2} \mathrm{H}$. Vrtilek et al. (1985) detected the $\mathrm{C}_{2} \mathrm{D}(N=$ $2-1)$ transition near the Orion-KL position, and obtained the column density $\sim 1.8 \times 10^{13} \mathrm{~cm}^{-2}$. Moreover, they derived the $N\left(\mathrm{C}_{2} \mathrm{D}\right) / N\left(\mathrm{C}_{2} \mathrm{H}\right)$ ratio of 0.05 . These are very similar to what we have found. Quite similarly, Parise et al. (2009) found upper limits of $N\left(\mathrm{C}_{2} \mathrm{D}\right)<2.5 \times 10^{13} \mathrm{~cm}^{-2}$ and $x\left(\mathrm{C}_{2} \mathrm{D}\right)<2 \times 10^{-10}$ in a clump associated with the Orion Bar.

The formation of $\mathrm{C}_{2} \mathrm{D}$ is believed to take place in the gas phase through the route $\mathrm{CH}_{3}^{+} \rightarrow \mathrm{CH}_{2} \mathrm{D}^{+} \rightarrow \mathrm{C}_{2} \mathrm{D}$ (see, e.g., Parise et al. 2009). The $N\left(\mathrm{C}_{2} \mathrm{D}\right) / N\left(\mathrm{C}_{2} \mathrm{H}\right)$ ratio we have obtained is comparable with those predicted by the low-metal abundance model by Roueff et al. (2007) at temperature around 30-40 K. We note that the $\mathrm{C}_{2} \mathrm{H}$ abundance, $\sim 6 \times 10^{-9}$, calculated from the observed deuteration degree (0.07) is comparable to the values $x\left(\mathrm{C}_{2} \mathrm{H}\right)=2.5 \times 10^{-9}-5.3 \times 10^{-8}$ recently found by Vasyunina et al. (2011) towards IRDCs.

Acrylonitrile or vinyl cyanide, ${ }^{15} \mathrm{~N}$ isotopologue $\left(\mathrm{CH}_{2} \mathrm{CHC}^{15} \mathrm{~N}\right)$. The Weeds modelling suggests that there is a $\mathrm{CH}_{2} \mathrm{CHC}^{15} \mathrm{~N}$ transition blended with the hf group of the detected $\mathrm{C}_{2} \mathrm{D}$ line. Thus, reliable column density estimate cannot be performed. Assuming that the detected line is completely due to $\mathrm{CH}_{2} \mathrm{CHC}^{15} \mathrm{~N}$ emission, we derive very high values of $2.5 \times 10^{16} \mathrm{~cm}^{-2}$ and $\sim 7 \times 10^{-7}$ for the column density and fractional abundance. For comparison, a column-density upper limit of $3 \times 10^{15} \mathrm{~cm}^{-2}$ towards Sgr B2(N) was derived by Müller et al. (2008).

If present, this molecule would indicate a hot-core chemistry. The main isotopologue $\mathrm{C}_{2} \mathrm{H}_{3} \mathrm{CN}$ is expected to form through gas-phase reactions after the ethyl cyanide $\left(\mathrm{C}_{2} \mathrm{H}_{5} \mathrm{CN}\right)$, forming on dust grains, evaporates into the gas phase (Caselli et al. 1993).

Acetone $\left(\mathrm{CH}_{3} \mathrm{COCH}_{3}\right)$. The first detection of $\mathrm{CH}_{3} \mathrm{COCH}_{3}$ in the interstellar medium was made by Combes et al. (1987) towards Sgr B2. They found the column density and fractional abundance of this molecule to be $5 \times 10^{13} \mathrm{~cm}^{-2}$ and $5 \times 10^{-11}$. These are significantly lower than we have estimated towards I18182 MM2 $\left(2 \times 10^{15} \mathrm{~cm}^{-2}\right.$ and $\left.\sim 4 \times 10^{-7}\right)$. Combes et al. (1987) found that the $\mathrm{CH}_{3} \mathrm{COCH}_{3}$ abundance is about $1 / 15$ of its precursor molecule $\mathrm{CH}_{3} \mathrm{CHO}$ (acetaldehyde), and suggested the formation route of acetone to be $\mathrm{CH}_{3}^{+}+\mathrm{CH}_{3} \mathrm{CHO} \rightarrow$ $\left(\mathrm{CH}_{3}\right)_{2} \mathrm{CHO}^{+}+h v ;\left(\mathrm{CH}_{3}\right)_{2} \mathrm{CHO}^{+}+\mathrm{e}^{-} \rightarrow \mathrm{CH}_{3} \mathrm{COCH}_{3}+\mathrm{H}$. However, Herbst et al. (1990) showed that this radiative association reaction is likely too slow to be consistent with the observed abundance in Sgr B2. The chemistry behind the formation of acetone is not clear. It could be caused by some other gas-phase ion-molecule reactions, or caused by grain chemistry (Herbst et al. 1990). 
Friedel et al. (2005) found that acetone in Orion BN/KL is concentrated towards the hot core. They derived the beamaveraged column densities of $\sim 2-8 \times 10^{16} \mathrm{~cm}^{-2}$. More recently, Goddi et al. (2009) found the column density $N\left(\mathrm{CH}_{3} \mathrm{COCH}_{3}\right)=$ $5.5 \times 10^{16} \mathrm{~cm}^{-2}$ in Orion BN/KL, in agreement with the Friedel et al. results. The very high column densities and abundances of $\mathrm{CH}_{3} \mathrm{COCH}_{3}$ found in Orion BN/KL and in I18182 MM2 in the present work require some other reaction pathway(s) than the above radiative association reaction to be efficient. Grain surface and hot-core gas-phase chemistry may both play critical roles (Garrod et al. 2008).

The detection of complex molecules in clumps associated with IRDCs, indicating the presence of hot cores, supports the scenario that high-mass star formation can take place in these objects. For example, Rathborne et al. $(2007,2008)$ found that the clumps G024.33+00.11 MM1 and G034.43+00.24 MM1 are both likely to contain a hot molecular core. On the other hand, complex organics could also be ejected from grain mantles through shocks, and cosmic rays heating up the dust can also have some effect (e.g., Requena-Torres et al. 2008, and references therein).

\section{Appendix B: Discussion on individual sources}

\section{B.1. IRDC 18102-1800 MM1}

The clump I18102 MM1 is the warmest $(21.3 \mathrm{~K})$ and most massive $\left(\sim 36 M_{\odot}\right)$ source of our sample. It is associated with Spitzer point sources at 8 and $24 \mu \mathrm{m}$, and high-mass star formation is taking place within it as indicated by the presence of the $6.7 \mathrm{GHz}$ Class II $\mathrm{CH}_{3} \mathrm{OH}$ maser (Beuther et al. 2002b). Among our sample, the lowest degree of deuteration in both $\mathrm{HCO}^{+}$and $\mathrm{N}_{2} \mathrm{H}^{+}$is found for I18102 MM1. On the other hand, the source shows the highest degree of ionisation.

Fuller et al. (2005) detected central dips and red asymmetries in the spectral lines $\mathrm{HCO}^{+}(1-0), \mathrm{HCO}^{+}(4-3), \mathrm{N}_{2} \mathrm{H}^{+}(1-0)$, and $\mathrm{H}_{2} \mathrm{CO}\left(2_{1,2}-1_{1,1}\right)$. A similar line profile is seen in the $J=3-2$ transition of $\mathrm{N}_{2} \mathrm{H}^{+}$in the present study. These profiles indicate the presence of expanding or outflowing gas (see, e.g., Park et al. 2000 and references therein). Beuther \& Sridharan (2007) detected very broad $\left(42.2 \mathrm{~km} \mathrm{~s}^{-1}\right.$ down to zero intensity) $\mathrm{SiO}(2-1)$ wings towards $\mathrm{I} 18102 \mathrm{MM} 1$, indicative of bipolar outflows. This source showed the second-broadest $\mathrm{SiO}$ line in the sample of Beuther \& Sridharan (2007). Also, the $\mathrm{SiO}(2-1)$ line detected by SSH10 towards I18102 MM1 was very wide, $\Delta v=13 \pm 1 \mathrm{~km} \mathrm{~s}^{-1}$. The $\mathrm{SiO}(6-5)$ line detected in the present study has $\Delta v \simeq 4.7 \mathrm{~km} \mathrm{~s}^{-1}$, and the width down to zero intensity is $\sim 10 \mathrm{~km} \mathrm{~s}^{-1}$. We have also detected the ${ }^{13} \mathrm{C}$ isotopologue of CS in this source. Sakai et al. (2010) suggested that CS could originate in the shock evaporation of grain mantles or radiative heating.

Beuther \& Sridharan (2007) detected $\mathrm{CH}_{3} \mathrm{CN}\left(6_{K}-5_{K}\right)$ and $\mathrm{CH}_{3} \mathrm{OH}\left(5_{K}-4_{K}\right)$ lines towards $\mathrm{I} 18102 \mathrm{MM} 1$, and derived the abundances of $3 \times 10^{-11}$ and $4 \times 10^{-11}$ for $\mathrm{CH}_{3} \mathrm{CN}$ and $\mathrm{CH}_{3} \mathrm{OH}$, respectively. Sakai et al. (2008) detected $\mathrm{CH}_{3} \mathrm{OH}\left(7_{K}-6_{K}\right)$ and $\mathrm{HC}_{3} \mathrm{~N}(5-4)$ lines in this source, and $\mathrm{SSH} 10$ detected $\mathrm{C}_{2} \mathrm{H}(\mathrm{N}=$ $1-0)$ and a hint of $\mathrm{CH}_{3} \mathrm{OH}\left(2_{1}-1_{1}\right) \mathrm{A}^{-}$. We note that SSK08 and SSH10 did not detected the lines of $\operatorname{CCS}\left(4_{3}-3_{2}\right), \operatorname{SO}\left(2_{2}-1_{1}\right)$, or OCS $(8-7)$ in their surveys. The upper limit they derived for the OCS column density, $\lesssim 2.7 \times 10^{14} \mathrm{~cm}^{-2}$, is about 8.5 times less than the $N(\mathrm{OCS})$ value we obtained. The observational results cumulated so far indicate hot-core chemistry in I18102 MM1. This conforms to the fact that this clump is giving birth to a highmass star(s), and possibly through disk accretion as indirectly suggested by the outflow signatures. The associated hot core is likely to be in its later stages of evolution because it is associated with a methanol maser (cf. Rathborne et al. 2008).

\section{B.2. G015.05+00.07 MM1 and G015.31-00.16 MM3}

G015.05 MM1 is the lowest mass $\left(63 M_{\odot}\right)$ and G015.31 MM3 is the second-coldest $(13.7 \mathrm{~K})$ clump of our sample. Both clumps are dark in the Spitzer 8 and $24 \mu \mathrm{m}$ images. The highest deuteration degree in $\mathrm{N}_{2} \mathrm{H}^{+}(0.028)$ was found towards G015.05 MM1, whereas G015.31 MM3 shows the largest CO depletion factor (2.7) in our sample. G015.05 MM1 is associated with $\mathrm{H}_{2} \mathrm{O}$ maser (Wang et al. 2006), indicative of star-formation activity. Rathborne et al. (2010) derived the following properties for G015.05 MM1 from the broadband SED: $T_{\text {dust }}=11-36 \mathrm{~K}$, $L=15.5-362 L_{\odot}$, and $M=35-158 M_{\odot}$ (scaled to the revised distance $2.6 \mathrm{kpc}$ ). The values $T_{\text {kin }}=17.2 \mathrm{~K}$ and $M=63 M_{\odot}$ derived in the present study lie at the low end of the above values of temperature and mass (at the derived clump densities, it is expected that $T_{\text {kin }} \simeq T_{\text {dust }}$, Goldsmith \& Langer 1978).

Sakai et al. (2008) barely detected the $\mathrm{HC}_{3} \mathrm{~N}(5-4)$, $\mathrm{CH}_{3} \mathrm{OH}\left(7_{K}-6_{K}\right)$, and $\mathrm{CCS}\left(4_{3}-3_{2}\right)$ lines towards G015.05 MM1 and $\mathrm{G} 015.31$ MM3. Indeed, all the $\mathrm{CH}_{3} \mathrm{OH}$ detected objects in the survey by SSK08 are associated with the Spitzer $24-\mu \mathrm{m}$ sources.

Both G015.05 MM1 and G015.31 MM3 are likely to be in a very early stage of evolution. Moreover, both sources are massive enough to allow high-mass star formation. G015.31 MM3 could represent or host the so-called high-mass prestellar core, whereas G015.05 MM1 could be slightly more evolved with $\mathrm{H}_{2} \mathrm{O}$ maser emission but still lacking IR emission at 8 and $24 \mu \mathrm{m}$. The small wave-coupling number of $W \sim 2$ for G015.31 MM3 suggests that "magnetic turbulence" is not able to fragment the clump into smaller pieces, strengthening the possibility that it is a massive prestellar "core".

\section{B.3. IRDC 18151-1208 MM2}

The I18151 MM2 clump is dark in the Spitzer 8$\mu \mathrm{m}$ image (24 $\mu \mathrm{m}$ not available). It is associated with $\mathrm{H}_{2} \mathrm{O}$ (Beuther et al. 2002b) and Class I $\mathrm{CH}_{3} \mathrm{OH}$ masers (Marseille et al. 2010b). This clump has the highest volumeaveraged $\mathrm{H}_{2}$ number density $\left(\sim 1.9 \times 10^{5} \mathrm{~cm}^{-3}\right)$ among our sources.

Beuther \& Sridharan (2007) detected the broadest $\mathrm{SiO}(2-1)$ wing emission (65 $\mathrm{km} \mathrm{s}^{-1}$ down to zero intensity) towards I18151 MM2 in their sample. Also, the $J=2-1$ and 3-2 SiO lines detected recently by López-Sepulcre et al. (2011) are very broad, i.e., FWZP $=84.3$ and $103.1 \mathrm{~km} \mathrm{~s}^{-1}$, respectively. The $\mathrm{SiO}(6-5)$ line we detected is also very broad with the FWHM $46.8 \mathrm{~km} \mathrm{~s}^{-1}$. The outflow activity within the clump was confirmed by Marseille et al. (2008) who, for the first time, found that I18151 MM2 is driving a CO outflow and hosts a mid-IRquiet, possibly a Class 0-like HMYSO (cf. Motte et al. 2007). Marseille et al. (2008) modelled the dust continuum emission (SED) of I18151 MM2 and found that the bolometric luminosity, mass, and the mean temperature of the source are $L_{\mathrm{bol}}=$ $2190 L_{\odot}, M_{\text {gas }}=373 \pm 81 M_{\odot}$, and $\langle T\rangle=19.4 \pm 0.2 \mathrm{~K}$ (scaled to the revised distance $2.7 \mathrm{kpc}$ ). Within the errors, these mass and temperature values are comparable to the values derived in the present paper. Besides the Class I methanol maser tracing the molecular outflow, Marseille et al. (2010b) detected blue asymmetry in $\mathrm{CH}_{3} \mathrm{OH}\left(5_{-1,5}-4_{0,4}\right) \mathrm{E}$, indicating infall motions. The 
$22 \mathrm{GHz} \mathrm{H}_{2} \mathrm{O}$ maser is probably excited in the outflow shocked gas (cf. Furuya et al. 2011).

Beuther \& Sridharan (2007) also detected $\mathrm{CH}_{3} \mathrm{CN}\left(6_{K}-5_{K}\right)$ and $\mathrm{CH}_{3} \mathrm{OH}\left(5_{K}-4_{K}\right)$ lines towards I18151 MM2, which is a sign of hot-core chemistry, and they derived the abundances of $8 \times 10^{-11}$ and $6 \times 10^{-10}$ for $\mathrm{CH}_{3} \mathrm{CN}$ and $\mathrm{CH}_{3} \mathrm{OH}$, respectively. SSK08 detected $\mathrm{CH}_{3} \mathrm{OH}\left(7_{K}-6_{K}\right)$ lines towards I18151 MM2, but not $\mathrm{CCS}\left(4_{3}-3_{2}\right)$ or $\mathrm{HC}_{3} \mathrm{~N}(5-4)$. In their line survey, SSH10 detected $\mathrm{C}_{2} \mathrm{H}(N=1-0)$, but not $\mathrm{SO}\left(2_{2}-1_{1}\right)$, OCS(8-7), or $\mathrm{CH}_{3} \mathrm{OH}\left(2_{1}-1_{1}\right) \mathrm{A}^{-}$lines. The $\mathrm{C}_{2} \mathrm{H}$ column density they derived, $\sim 2.2 \times 10^{14} \mathrm{~cm}^{-2}$, together with the value $N\left(\mathrm{C}_{2} \mathrm{D}\right)=1.5 \times 10^{13}$ $\mathrm{cm}^{-2}$ derived by us, suggest a deuteration degree of $\sim 0.07$ in $\mathrm{C}_{2} \mathrm{H}$. Marseille et al. (2008) concluded from their modelling of CS transitions that CS is depleted in I18151 MM2. We derived only a small CO depletion factor of $\sim 1.6$ for this clump, and low degrees of deuteration in $\mathrm{HCO}^{+}$and $\mathrm{N}_{2} \mathrm{H}^{+}$, namely $0.3 \%$ and $1 \%$, respectively. Also, this source shows the lowest lower limit to ionisation degree, only $7 \times 10^{-9}$.

\section{B.4. IRDC 18182-1433 MM2}

The filamentary clump I18182 MM2 is associated with Spitzer 8 and $24-\mu \mathrm{m}$ sources. This source shows the highest degree of deuteration in $\mathrm{HCO}^{+}(0.014)$.

Beuther \& Sridharan (2007) detected $\mathrm{CH}_{3} \mathrm{OH}\left(5_{K}-4_{K}\right)$ lines towards I18182 MM2, and derived the $\mathrm{CH}_{3} \mathrm{OH}$ abundance of $2.1 \times 10^{-10}$. Sakai et al. (2008) did not detect $\operatorname{CCS}\left(4_{3}-3_{2}\right)$, $\mathrm{CH}_{3} \mathrm{OH}\left(7_{K}-6_{K}\right)$, or $\mathrm{HC}_{3} \mathrm{~N}(5-4)$ lines towards this source in their survey. Our tentative detection of the O-bearing species $\mathrm{CH}_{3} \mathrm{COCH}_{3}$ indicates hot-core chemistry within the clump. Moreover, O-bearing species are sign of the early stage of chemical evolution (e.g., Shiao et al. 2010). This conforms to the presumable young age of the clump as it is associated with IRDC. Interestingly, the nearby clump I18182 MM1, which is associated with the HMYSO IRAS 18182-1433, also appears to contain a hot core (Beuther et al. 2006).

\section{B.5. IRDC 18223-1243 MM3}

I18223 MM3, part of a long filamentary IRDC, is a high-mass clump harbouring an embedded accreting low- to intermediatemass protostar that could evolve to a high-mass star at some point in the future (see Beuther \& Steinacker 2007; Beuther et al. 2010). Fallscheer et al. (2009) detected a molecular outflow in this source and found evidence for a large rotating structure, or toroid, perpendicular to the outflow. Evidence for outflow activity in this source was already found by Beuther et al. (2005c) and Beuther \& Sridharan (2007), who found that there are $4.5 \mu \mathrm{m}$ emission features at the clump edge and that the spectral lines of $\mathrm{CO}, \mathrm{CS}$, and $\mathrm{SiO}$ show broad wing emission. The line profile of $\mathrm{SiO}(6-5)$ detected in the present study also indicates outflowing gas.

The clump shows $24 \mu \mathrm{m}$ emission but is dark at the Spitzer IRAC wavelengths $(3.6,4.5,5.8$, and $8.0 \mu \mathrm{m})$. Beuther et al. (2010) derived an SED for this source between $24 \mu \mathrm{m}$ and $1.2 \mathrm{~mm}$, including the recent Herschel PACS and SPIRE data, and obtained the total luminosity of $539 L_{\odot}$ (scaled to $d=$ $3.5 \mathrm{kpc}$ ). They also found that the ratio between the total and submm luminosity (integrated longward of $400 \mu \mathrm{m}$ ) is only 11 , suggesting that the source is very young.

Beuther \& Sridharan (2007) detected $\mathrm{CH}_{3} \mathrm{CN}\left(6_{K}-5_{K}\right)$ and $\mathrm{CH}_{3} \mathrm{OH}\left(5_{K}-4_{K}\right)$ lines towards I18223 MM3, indicative of hot-core chemistry, and derived the abundances of $9 \times 10^{-11}$ and $6.1 \times 10^{-10}$ for $\mathrm{CH}_{3} \mathrm{CN}$ and $\mathrm{CH}_{3} \mathrm{OH}$, respectively. Sakai et al. (2008) detected $\mathrm{HC}_{3} \mathrm{~N}(5-4)$, only weak $\mathrm{CH}_{3} \mathrm{OH}\left(7_{K}-6_{K}\right)$ lines, and no $\operatorname{CCS}\left(4_{3}-3_{2}\right)$ lines towards this source. Sakai et al. (2010) did not detect the lines $\operatorname{SO}\left(2_{2}-1_{1}\right)$, OCS $(8-7)$, or $\mathrm{CH}_{3} \mathrm{OH}\left(2_{1}-1_{1}\right) \mathrm{A}^{-}$in their survey; however, they detected the $\mathrm{C}_{2} \mathrm{H}(N=1-0)$ line. Based on the column densities of different species (e.g., $\mathrm{SiO}$ and $\mathrm{H}^{13} \mathrm{CO}^{+}$), SSH10 suggested that $\mathrm{I} 18223$ MM3 is in early stage of evolution. This conforms to the fact that the second-highest value of $R_{\mathrm{D}}\left(\mathrm{N}_{2} \mathrm{H}^{+}\right)$in our sample $(0.013)$ is found towards I18223 MM3.

\section{B.6. ISOSS J18364-0221 SMM1}

At the distance of $\sim 2.5 \mathrm{kpc}$, the clump J18364 SMM1 is the nearest source in our sample. It is also the coldest (11.4 K) clump of our sample. The clump is dark at $8 \mu \mathrm{m}$ but it is associated with the $24-\mu \mathrm{m}$ point source. The second-highest value of $R_{\mathrm{D}}\left(\mathrm{HCO}^{+}\right)$ in our sample, 0.012 , is found towards this source. It also shows high cosmic-ray ionisation rate of $\mathrm{H}_{2}\left(\sim 5 \times 10^{-16} \mathrm{~s}^{-1}\right)$. Birkmann et al. (2006) studied this clump through $J=3-2$ transitions of $\mathrm{HCO}^{+}$and $\mathrm{H}^{13} \mathrm{CO}^{+}$(beam size $\sim 9.5$ ). The former line showed blue asymmetric profile, indicating infall. They also found significant $\mathrm{CO}(2-1)$ line wings, indicating the presence of outflows. The $\mathrm{H}^{13} \mathrm{CO}^{+}(3-2)$ line we observed shows an asymmetric profile with a central dip and slightly stronger red peak, contrary to that observed by Birkmann et al. (2006) in $\mathrm{HCO}^{+}(3-2)$. This difference is probably caused by the larger beam size of our observations (24"), yielding a signature of outflowing gas motions.

More recently, J18364 SMM1 was studied in detail by Hennemann et al. (2009). They resolved this contracting clump in the interferometric mm continuum into two compact cores, named SMM1 North and South separated by 9.'5 (0.12 pc). Their positions are indicated in Fig. 1. The peak $\mathrm{H}_{2}$ column densities and dust temperatures were found to be $2.7 \times 10^{23} \mathrm{~cm}^{-2}$ and $15 \mathrm{~K}$ for SMM1 North and, $2.4 \times 10^{23} \mathrm{~cm}^{-2}$ and $22 \mathrm{~K}$ for SMM1 South. Using the revised distance $2.5 \mathrm{kpc}$, the radius, mass, and luminosity of the northern core are $0.06 \mathrm{pc}, 19 M_{\odot}$, and $26 L_{\odot}$, whereas for the southern core these values are $0.05 \mathrm{pc}, 13 M_{\odot}$ and $230 L_{\odot}$. Thus, the cores within the clump have comparable sizes and masses but the southern one is associated with the Spitzer 24 and $70 \mu \mathrm{m}$ sources and is more luminous. Hennemann et al. (2009) found that SMM1 South drives an energetic molecular outflow and that the core centre is supersonically turbulent. On the other hand, the IR-dark core SMM1 North shows lower levels of turbulence, but it also drives an outflow. Both the outflows from SMM1 North and South are quite collimated and their estimated ages are $<10^{4} \mathrm{yr}$. The $\mathrm{HCN}(1-0)$ modelling results by Hennemann et al. (2009) showed that the spectrum of SMM1 South can be explained with a collapse of the core. They obtained an infall velocity of $0.14 \mathrm{~km} \mathrm{~s}^{-1}$ and an estimated mass infall rate of $\sim 3.4 \times 10^{-5} M_{\odot} \mathrm{yr}^{-1}$ (scaled to the revised distance).

In summary, the clump J18364 SMM1 is fragmented into two cores that both harbour protostellar seeds, possibly evolving into intermediate- to high-mass stars. As discussed in Sect. 5.4, MHD wave propagation could have played a role in fragmenting the parent clump. As the southern core harbours a $24 \mu \mathrm{m}$ source, is highly turbulent in the central region, and shows jet features at large distance from the driving source, it appears to be more evolved than the northern core. 